NBS

PUBLICATIONS

$\therefore$ Q

All102 ?06636

NATL INST OF STANDARDS \& TECH RIC

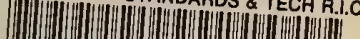

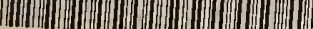

A11102706636

NBS TECHNICAL NOTE 1311

U.S. DEPARTMENT OF COMMERCE / National Bureau of Standards

Extrapolation Range Measurements for Determining Antenna Gain and Polarization

\author{
Andrew G. Repjar \\ Allen C. Newell \\ Douglas T. Tamura
}

$Q C$

100

.45753

No.1311

1987

C.2 
he National Bureau of Standards' ${ }^{1}$ was established by an act of Congress on March 3, 1901. The Bureau's overall goal is to strengthen and advance the nation's science and technology and facilitate their effective application for public benefit. To this end, the Bureau conducts research to assure international competitiveness and leadership of U.S. industry, science ard technology. NBS work involves development and transfer of measurements, standards and related science and technology, in support of continually improving U.S. productivity, product quality and reliability, innovation and underlying science and engineering. The Bureau's technical work is performed by the National Measurement Laboratory, the National Engineering Laboratory, the Institute for Computer Sciences and Technology, and the Institute for Materials Science and Engineering.

\section{The National Measurement Laboratory}

Provides the national system of physical and chemical measurement; coordinates the system with measurement systems of other nations and furnishes essential services leading to accurate and uniform physical and chemical measurement throughout the Nation's scientific community, industry, and commerce; provides advisory and research services to other Government agencies; conducts physical and chemical research; develops, produces, and distributes Standard Reference Materials; provides calibration services; and manages the National Standard Reference Data System. The Laboratory consists of the following centers:
- Basic Standards ${ }^{2}$

- Radiation Research

- Chemical Physics

- Analytical Chemistry

\section{The National Engineering Laboratory}

Provides technology and technical services to the public and private sectors to address national needs and to solve national problems; conducts research in engineering and applied science in support of these efforts; builds and maintains competence in the necessary disciplines required to carry out this research and technical service; develops engineering data and measurement capabilities; provides engineering measurement traceability services; develops test methods and proposes engineering standards and code changes; develops and proposes new engineering practices; and develops and improves mechanisms to transfer results of its research to the ultimate user. The Laboratory consists of the following centers:

\section{The Institute for Computer Sciences and Technology}

Conducts research and provides scientific and technical services to aid Federal agencies in the selection, acquisition, application, and use of computer technology to improve effectiveness and economy in Government operations in accordance with Public Law 89-306 (40 U.S.C. 759), relevant Executive Orders, and other directives; carries out this mission by managing the Federal Information Processing Standards Program, developing Federal ADP standards guidelines, and managing Federal participation in ADP voluntary standardization activities; provides scientific and technological advisory services and assistance to Federal agencies; and provides the technical foundation for computer-related policies of the Federal Government. The Institute consists of the following divisions:
- Applied Mathematics

- Electronics and Electrical Engineering ${ }^{2}$

- Manufacturing Engineering

- Building Technology

- Fire Research

- Chemical Engineering ${ }^{3}$

\section{The Institute for Materials Science and Engineering}

Conducts research and provides measurements, data, standards, reference materials, quantitative understanding and other technical information fundamental to the processing, structure, properties and performance of materials; addresses the scientific basis for new advanced materials technologies; plans research around cross-cutting scientific themes such as nondestructive evaluation and phase diagram development; oversees Bureau-wide technical programs in nuclear reactor radiation research and nondestructive evaluation; and broadly disseminates generic technical information resulting from its programs. The Institute consists of the following Divisions:
- Information Systems Engineering

- Systems and Software Technology

- Computer Security

- Systems and Network Architecture

- Advanced Computer Systems 


\title{
Extrapolation Range Measurements for Determining Antenna Gain and Polarization
}

\author{
Andrew G. Repjar \\ Allen C. Newell \\ Douglas T. Tamura
}

Electromagnetic Fields Division

Center for Electronics and Electrical Engineering

National Engineering Laboratory

National Bureau of Standards

Boulder, Colorado 80303-3328

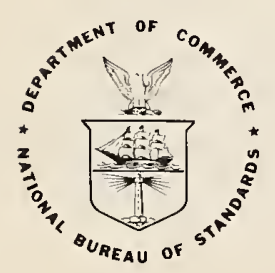

U.S. DEPARTMENT OF COMMERCE, Clarence J. Brown, Acting Secretary NATIONAL BUREAU OF STANDARDS, Ernest Ambler, Director 
National Bureau of Standards Technical Note 1311

Natl. Bur. Stand. (U.S.), Tech Note 1311, 88 pages (Aug. 1987) CODEN:NBTNAE

U.S. GOVERNMENT PRINTING OFFICE WASHINGTON: 1987

For sale by the Superintendent of Documents, U.S. Government Printing Office, Washington, DC 20402 


\section{Contents}

Page

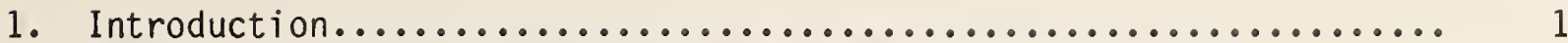

2. Definitions and Basic Concepts from a Measurement

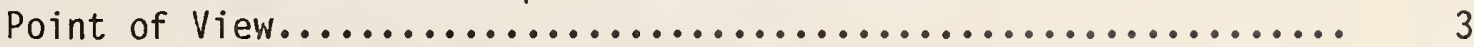

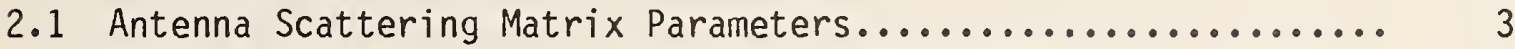

2.2 Generalization of the Three-Antenna Measurement

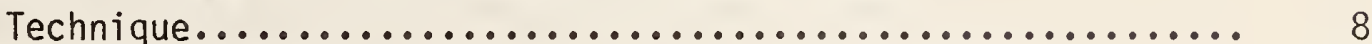

2.3 Measurement Methods for the Extrapolation Technique......... 14

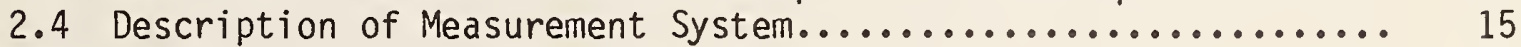

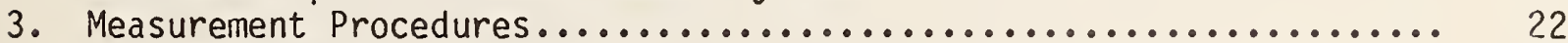

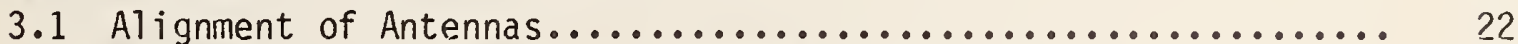

3.2 Receiver Calibration and Determination of $a_{0} \ldots \ldots \ldots \ldots \ldots . \ldots 23$

3.3 Amplitude, Phase and Distance Data, General.............. 27

3.4 Amplitude Measurements........................... 27

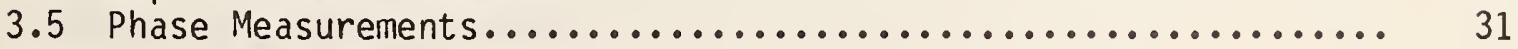

4. Numerical Techniques for Antenna Gain and Polarization

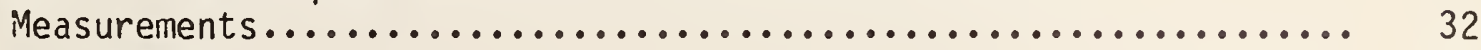

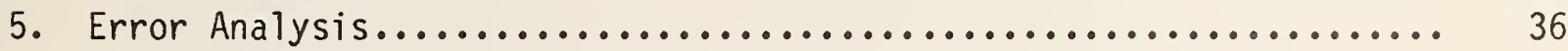

5.1 Propagation of Errors to Gain and Polarization

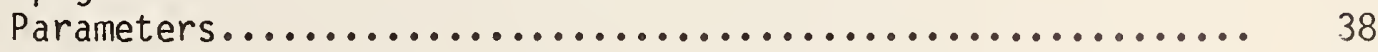

5.2 Three Nominally Linearly Polarized Antennas................ 39

5.3 Two Nominally Linearly and One Nominally Circularly

Polarized Antenna Combination....................... 40

6. Measurement Examples.................................. 42

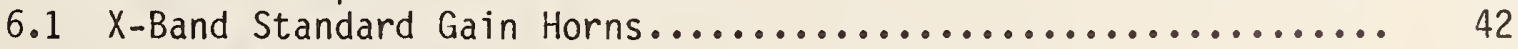

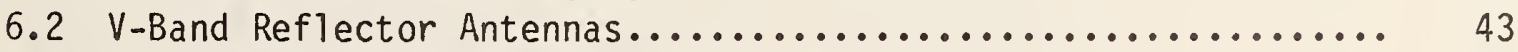

7. Improved Polarization Measurements......................... 44

8. Swept Frequency Measurement Techniques....................... 50

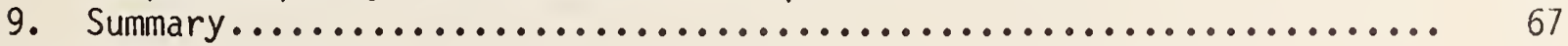

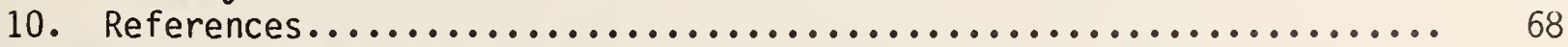

Appendix A. Extension of Extrapolation Technique to Correct

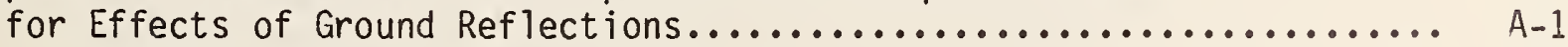

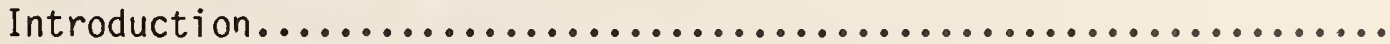

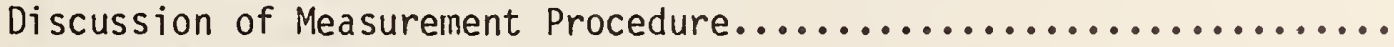

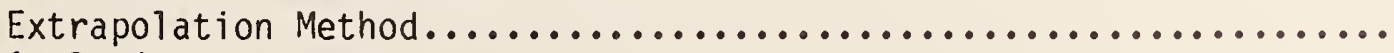

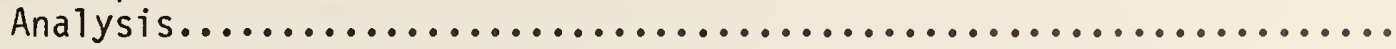

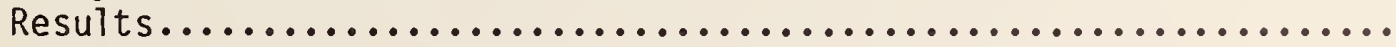

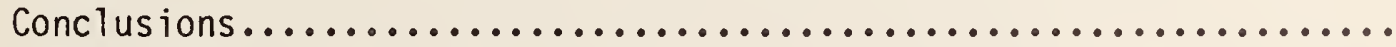

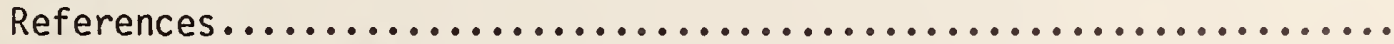

Appendix 3. Standard NBS Calibration Report..................... 



\section{List of Figures}

Figure

Page

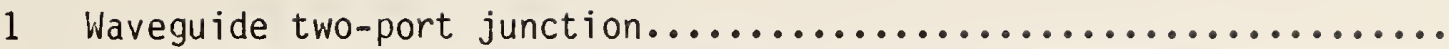

Plane-wave scattering-matrix description of an antenna..........

Schematic of two antennas oriented for measurement..............

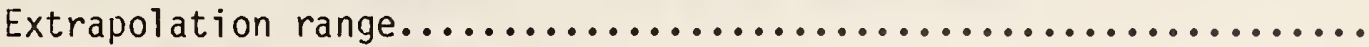

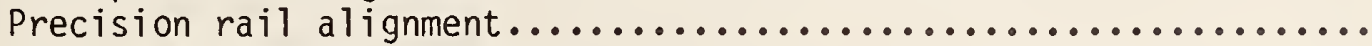

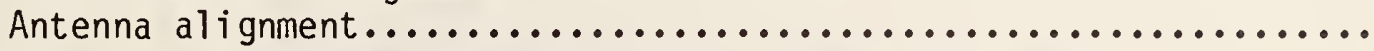

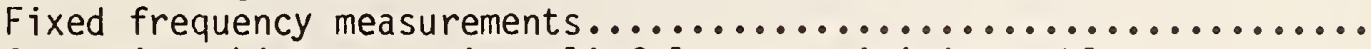

8 Steps in making a strain relief loop to minimize cable

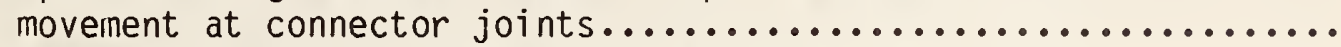

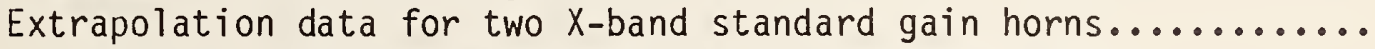

10 Extrapolation data showing multipath interference and averaged curve for two $45 \mathrm{~cm}$ (18 in), millimeter-wave,

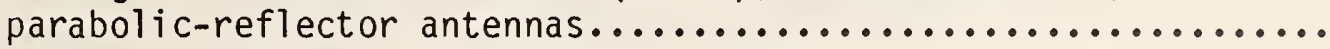

11 Averaged extrapolation data for the same antenna as

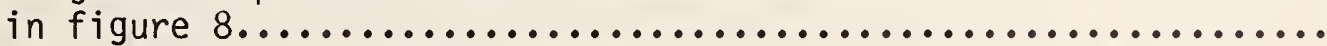

12 Measured phase difference between $b_{0}^{\prime}$ and $b_{0}^{\prime \prime}$ plotted as a function of distance for a $1.2 \mathrm{~m}(4 \mathrm{ft})$ diameter

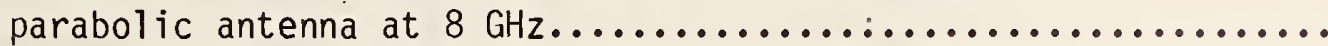

Measured-data, two-term polynomial $\mathrm{fit}$, and residuals for $X$-band conical horn (gain $\approx 22 \mathrm{~dB}) \ldots \ldots \ldots \ldots \ldots \ldots \ldots \ldots . . . \ldots$

14 Measured-data, five-term polynomial fit, and residuals for $X$-band conical horn (gain $\approx 22 \mathrm{~dB}) \ldots \ldots \ldots \ldots \ldots \ldots \ldots \ldots \ldots$

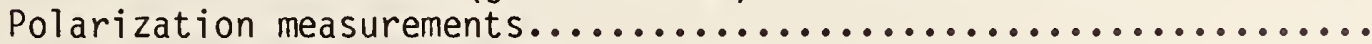

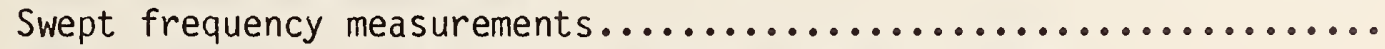

Swept frequency, reflection coefficient measurement

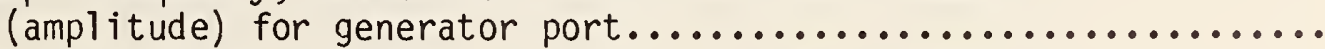

18 Swept frequency, reflection coefficient measurement

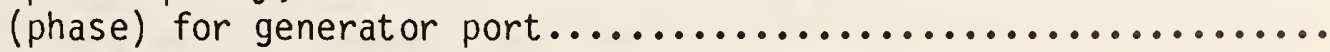

19 Swept frequency, reflection coefficient measurement (amplitude) for a long pyramidal horn.....................

Swept frequency, reflection coefficient measurement (phase) for a long pyramidal horn.......................

21 Mismatch correction calculated from measured

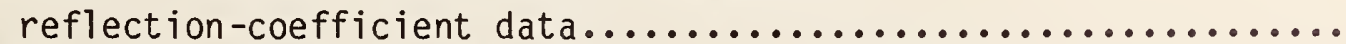

Near-zone correction obtained from extrapolation method for fixed-frequency measurements at $11.7,14.5$,

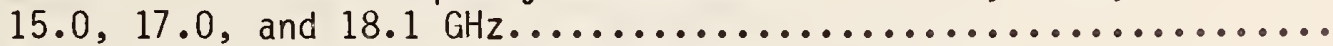

23 Swept frequency amplitude measurement with antennas in place at fixed distance of about $a^{2} / \lambda \ldots \ldots \ldots \ldots \ldots \ldots \ldots . . . . .$.

Swept frequency reference amplitude curve obtained with generator and load ports connected directly together........... nsertion loss including mismatch and near-zone corrections. This is the difference between the reference curve and amplitude curve with antennas in place. The high-frequency oscillations are due to multiple reflec-

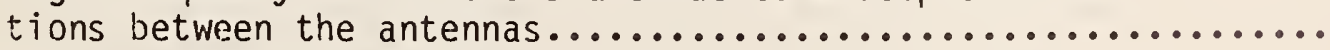


28 Final gain values for a long Ku-band horn. The oscillations about the nominal values are approximately $\pm 0.04 \mathrm{~dB}$ and are due to mismatches at the throat and

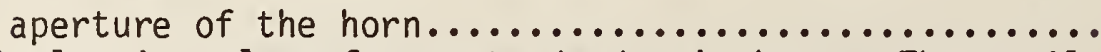

29 Final gain values for a standard gain horn. The oscil-

lations about the nominal values are approximately $\pm 0.1 \mathrm{~d} B$ and are due to mismatches at the throat and

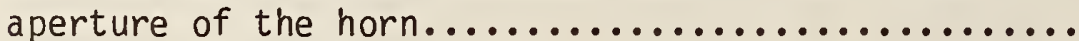

30 Comparison between theoretical gain and gain measured by the swept frequency technique for an $X$-band pyramidal horn. Gain values indicated by the * were obtained by the extrapolation method...

\section{List of Tables}

Table

Page

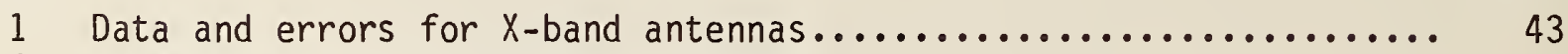

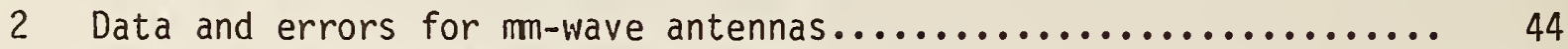

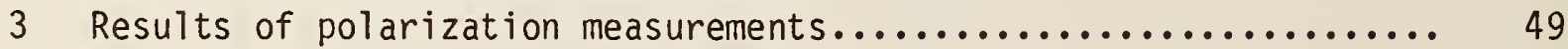


Extrapolation Range Measurements for Determining Antenna Gain and Polarization

Andrew G. Repjar, Allen C. Newell, and Douglas T. Tamura

National Bureau of Standards

Electromagnetic Fields Division

Boulder Colorado 80303

The extrapolation range measurement technique for determining the power gain and polarization of antennas at reduced range distances is described. It is based on a generalized three-antenna approach and does not require quantitative a priori knowledge of the antennas. During the past decade, it has been extensively used by the National Bureau of Standards, Boulder, Colorado, to calibrate antenna gain standards for industry and other agencies within $\pm 0.1 \mathrm{~dB}$. To help one understand how calibrations of this accuracy are achieved, the extrapolation range description includes discussions on the required theory, the measurement procedures, the range configuration and instrumentation, the errors, and some measurement examples. Recent extensions of the extrapolation method required for swept/stepped frequency gain calibrations and for corrections to reduce ground reflection effects, are al so presented.

Key words: antenna calibrations; antenna measurements; antenna ranges; antennas; near-field measurements; probe antennas; standard gain horns; swept frequency measurements

\section{Introduction}

Accurate antenna gain standards are necessary to evaluate and verify the performance of communication, radar, navigation, remote sensing, and other systems that transmit or receive radiated electromagnetic energy. Until the mid-1970s, the most common method of measuring the antenna gain and polarization for these systems was a substitution technique, where the response of the test antenna to an incident plane-wave field was compared with that of one or more standard antennas. Since then, near-field measurement techniques, where a probe scans over a planar [1], cylindrical [2,3], and spherical [4,5] surface, have been extensively used. In either case, the characteristics of the standard antennas or probe must be determined. 
Computed gain and polarization values for a standard antenna do not provide a satisfactory solution for high accuracy measurements. The gain of pyramidal horn antennas, used extensively as standards or probes $[6,7]$ may be calculated within an estimated uncertainty of about $\pm 0.3 \mathrm{~dB}$. Similarly, gain calculations for both smooth and corrugated conical horns $[8,9]$ have about the same accuracy. The uncertainties in these calculated values are due to theoretical approximations and imperfect fabrication, problems not easily overcome. For this reason, the National Bureau of Standards (NBS) has opted to develop and employ "standard measurement methods" rather than "standard antennas." By these methods, an arbitrary antenna can be accurately calibrated for use as a gain and polarization transfer standard.

The well known, far-field three-antenna method is not suitable for accurate absolute measurements of many standard antennas for several reasons. With conventional far-field antenna ranges, the plane-wave condition is approximated by using large separation distances between the transmitting and receiving sites. However, for high gain antennas, ranges of sufficient length may not be available. This is especially true when high accuracy is desired. For example, to reduce the proximity correction of typical standard gain horns to less than $0.05 \mathrm{~dB}$ requires a distance of about 32 (not 2) $\mathrm{a}^{2} / \lambda$ [10], where a is the largest aperture dimension and $\lambda$ is the free-space wavelength. At such large distances the errors due to ground reflections and scattering from other objects can be significant. Additional errors can occur if the test and standard antennas have significantly different gains, patterns, or polarization characteristics. Interference from other signals, and (at millimeter-wave frequencies) strong atmospheric attenuation are other problems. To overcome these difficulties and provide a means of accurately characterizing antenna standards, NBS developed a new method, known as the extrapolation technique, for evaluating directive antennas. This method is not well suited for pattern measurements (although pattern data can be obtained), but it is the most accurate method known for determining absolute gain and polarization. (Gain is routinely obtained within $0.1 \mathrm{~dB}$.) Hence, it is most useful in evaluating standards and for measuring antennas when the highest accuracy is required. The theoretical basis was developed by Wacker [11], and its application to accurate antenna measurements has been described briefly by Newell and Kerns [12] and more fully in an experimentally oriented paper by Newell et al. [13]. 
The method uses a generalized three-antenna approach which does not require quantitative a priori knowledge of the antennas. It is the latter work that provides the basis for this updated and expanded exposition.

The main purpose of this technical note is to describe all aspects of the extrapolation measurement procedure used at NBS for antenna calibrations, in detail and in one convenient document. The treatise will include a discussion of the results of the scattering matrix theory [1] that are required in the development of the generalized three-antenna and extrapolation techniques, the measurement procedures including range configuration and instrumentation, the data processing, a discussion on errors, measurement examples, and a discussion on improved polarization measurements and swept frequency measurements. The extension of the extrapolation technique which corrects for the effects of ground reflections in absolute gain measurements is presented in Appendix $A$. An example of an NBS calibration report is then given in Appendix B. Through this exposition, it is intended that the reader will understand the deliberate steps taken to assure calibrations of the highest accuracy.

2. Definitions and Basic Concepts from a Measurement Point of View 2.1 Antenna Scattering Matrix Parameters

In this discussion frequent use of the quantities used in the scattering matrix theory of antennas which is more completely discussed el sewhere is made [1]. A brief discussion is given here to acquaint the reader with the basic quantities and their meaning, and to give the results of the theory that are required in the development of the three-antenna and extrapolation techniques. The intent of the following discussion is to describe the concepts used in this new approach to antenna theory in terms of quantities which should be familiar to the microwave and antenna engineer.

The theory was developed as an extension of the familiar scattering matrix theory of waveguide junctions and therefore much of the notation and formulation is similar. Because of this similarity we will begin the discussion by referring to a waveguide two-port shown in figure 1 , as it is described by the $S$ parameters. 


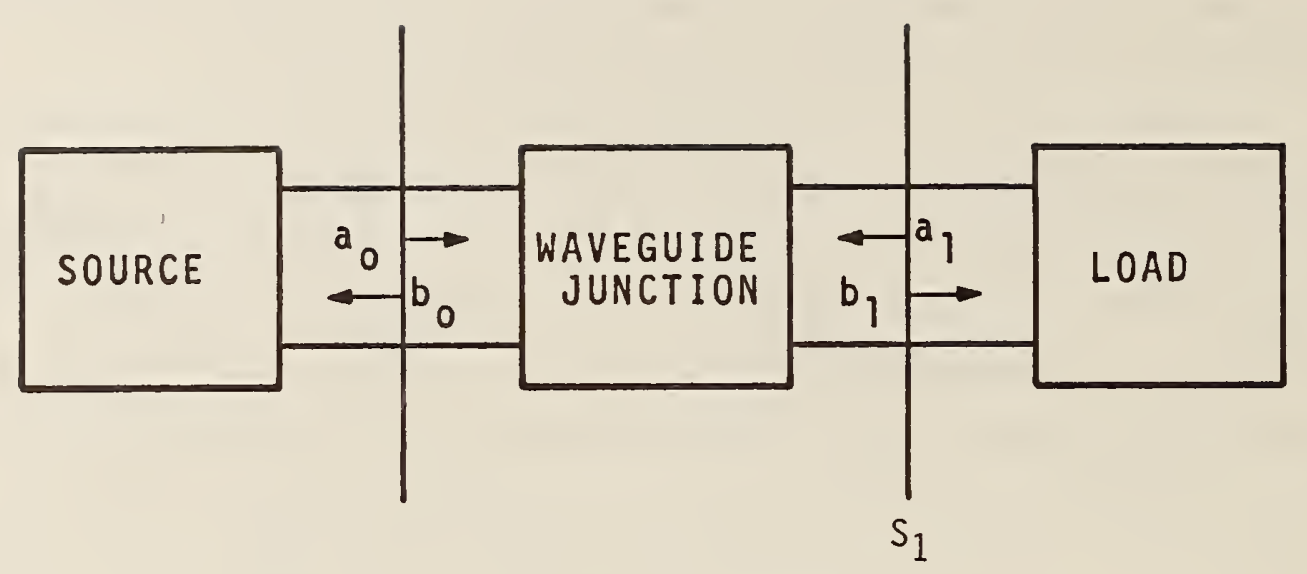

Figure 1. Waveguide two-port junction.

The incident and emergent wave amplitudes $a_{0}, a_{1}, b_{0}, b_{1}$ on the two terminal surfaces $S_{0}$ and $S_{1}$ are related by the equations [14],

$$
b_{0}=S_{00} a_{0}+S_{01} a_{1}
$$

and

$$
b_{1}=S_{10 a_{0}}+S_{11} a_{1}
$$

The $S$ parameters are complex scalar quantities which specify the response of this two-port transducer at a given frequency and for one waveguide mode. Once these quantities have been determined from measurements, it can be determined how this device will interact with others in a composite system.

Although an antenna is more complicated than a simple waveguide two-port, it can still be described by the same sort of formal representation. The antenna is viewed as a two-port transducer which transforms wave amplitudes in a closed transmission line system to an angular spectrum of plane waves in the space system. The $S$ parameters provide a powerful and yet simple means for a formal description of this transducer point of view. As with the simple twoport, once these $S$ parameters have been determined, they may be used to evaluate the antenna performance in a larger, more complicated system. Familiar quantities such as power gain and axial ratio are easily derived from the $S$ 


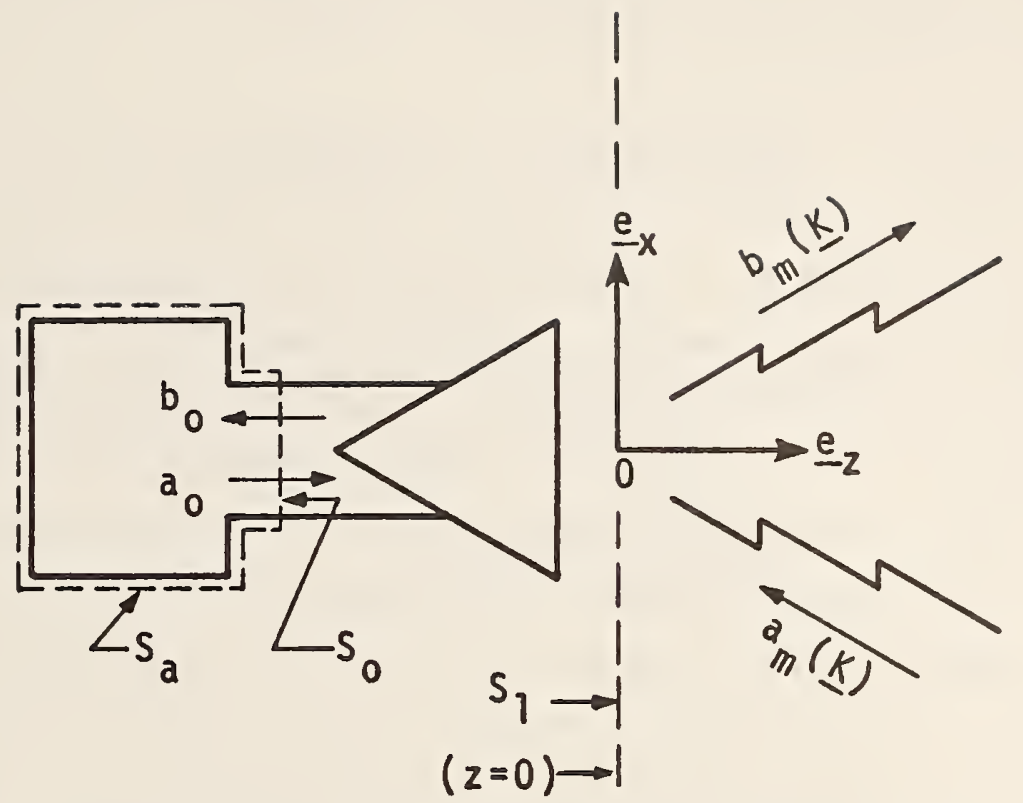

Figure 2. Plane-wave scattering-matrix description of an antenna.

parameters, but they are not limited to use in far-field plane-wave situations which are usually assumed. One of their main advantages is that they give a means of formulating near-field antenna problems in a way that gives new insight into the problems and allows practical solutions.

To further define these parameters, consider the antenna shown in figure 2 oriented in the coordinate system $0 x y z$ with unit vectors $e_{-x}, e_{-y}, e_{z}$ in the space to the right of the antenna, and let $\underline{k}$ be the propagation vector for plane waves in this space. Since the magnitude of $\underline{k}$ is fixed, $k^{2}=\omega^{2} \mu \varepsilon$, we can consider $k_{z}$ and also $\underline{k}$ to be functions of the transverse components $k_{x}$ and $k_{y}$ and define $\underline{k}=k_{x} e_{x}+k_{y} e_{-y} \cdot$ Then $k_{z}= \pm \gamma$, where

$$
\gamma=\sqrt{k^{2}-k^{2}}
$$

$\underline{K}$ will be the independent variable in most of what follows, and defines directions in the space to the right of the antenna. For example, $\underline{K}=0$ defines the reference boresight direction, $\underline{k}=k_{y} \underline{e}_{-y}+0 \quad e_{-x}$ defines an E-plane cut for an antenna with linear polarization in the $y$-direction, and $\underline{k}=0 \underline{e}_{-y}+k_{x} \underline{e}_{x}$ defines an H-plane cut for the same antenna. 
In addition to the unit vectors in the $x, y$, and $z$ directions, we define two additional vectors in the $x-y$ plane which are used in the formulation. These vectors, $\underline{\underline{k}}_{1}$ and $\underline{\underline{k}}_{2}$ are

$$
\underline{k}_{1}=\underline{k} /|\underline{k}| \quad \underline{k}_{2}=\underline{e}_{2} \times \underline{k}_{1} .
$$

In figure 2, $a_{0}$ and $b_{0}$ are incident and emergent wave amplitudes at the surface $S_{0}$ just as in the waveguide junction formulation. $a_{m}(\underline{K})$ and $b_{m}(\underline{K})$ are spectral density functions for incoming and outgoing $p l a n e$ waves and are analogous to $a_{0}$ and $b_{0}$. This decomposition of the transverse electric field into a continuous angular spectrum of plane waves is analogous to the familiar practice of analyzing a complicated time function in terms of its frequency components. Indeed, there are many concepts from time and frequency domain analys is which can be carried over to the antenna problem and give new insight to the processes involved. These ideas are formalized by the following equations [15] which define the S-parameter functions that characterize the antenna:

$$
b_{0}=S_{00} a_{0}+\int \sum_{m=1}^{2} S_{01}(m, \underline{K}) a_{m}(\underline{K}) d \underline{K},
$$

and

$$
b_{m}(\underline{K})=S_{10}(m, \underline{K}) a_{0}+\int \sum_{n=1}^{2} S_{11}(m, \underline{K} ; n, \underline{L}) a_{n}(\underline{L}) d \underline{L},
$$

where the summation is over two values of the polarization index $m$ or $n$, (corresponding to the two orthogonal polarizations, $\underline{k}_{1}$ and $\underline{k}_{2}$ of the wave in the $x-y$ plane), integration is over all values of $\underline{K}$ (or $\underline{L})$, and $\mathrm{dK}$ denotes the surface element for integration (e.g., $\left.d k_{x} d k_{y}\right)$.

The significance of each parameter can best be seen by considering two special cases. First assume that the antenna is transmitting into free space and therefore there are no waves incident from the right, $a_{m}(\underline{K})=0$. We then have

$$
b_{0}=S_{00} a_{0}
$$


and

$$
b_{m}(\underline{K})=S_{10}(m, \underline{K}) a_{0}
$$

$S_{00}$ is thus a simple input reflection coefficient term. $S_{10}(m, K)$ defines the transmitting characteristic since it describes how input wave amplitude $a_{0}$ is transformed by the antenna into an angular pattern of plane waves $b_{m}(\underline{K})$.

Next assume that the antenna is being used as a receiver with a matched load termination and therefore $a_{0}=0$. We now have

$$
b_{0}=\int \sum_{m=1}^{2} S_{01}(m, \underline{K}) a_{m}(\underline{K}) d \underline{M},
$$

and

$$
b_{m}(\underline{K})=\int \sum_{n=1}^{2} S_{11}(m, \underline{K} ; n, \underline{L}) a_{n}(\underline{L}) d \underline{.} .
$$

$S_{01}(m, K)$ is called the receiving characteristic since it describes how the antenna responds to an incident spectrum of plane waves $a_{m}(\underline{K}) \cdot S_{11}(m, \underline{K} ; n, \underline{L})$ is called the scattering characteristic as it describes how waves incident from the right are scattered back into the same space.

If the antenna is reciprocal, $S_{10}(m, \underline{K})$ and $S_{01}(m, \underline{K})$ are related by the reciprocity relation,

$$
-n_{0} S_{01}(m, \underline{K})=n_{m}(\underline{K}) S_{10}(m,-\underline{K}) \text {, }
$$

where $n_{1}=\omega \varepsilon / \gamma, n_{2}=\gamma /(\omega \mu)$ and $n_{0}=\left(\varepsilon_{0} / \mu_{0}\right)^{1 / 2}$, is the characteristic admittance of free space.

If we define the vectorial transmission spectrum as

$$
\underline{S}_{10}(\underline{K})=S_{10}(1, \underline{K}) \underline{K}_{1}+S_{10}(2, \underline{K}) \underline{K}_{2},
$$

then the transverse electric field at large distances $\underline{r}$ from the source is given by [15] 


$$
\underline{E}_{t}(\underline{r})=\frac{-2 \pi i k}{r} \cos \theta \underline{S}_{10}(\underline{R} k / r) a_{0} e^{i k r} \text {, }
$$

where $\underline{r}$ is the position vector with transverse part $\underline{R}, r=|r|$, and $\theta$ is the angle between $\underline{k}$ and $\underline{e}_{z}$, or equivalently the polar angle of $\underline{r}$ relative to the $z$-axis. The power gain function is also given in terms of $\underline{S}_{10}(\underline{K})$ as

$$
G(\underline{K})=\frac{4 \pi k^{2} \cos \theta\left[\left|S_{10}(1, K)\right|^{2} n_{1}+\left|S_{10}(2, K)\right|^{2} n_{2}\right]}{n_{0}\left(1-\left|S_{00}\right|^{2}\right)} .
$$

Since the electric field, the receiving characteristic for a reciprocal antenna, and the gain function can $a 11$ be found from $\underline{S}_{10}(\underline{K})$, the measurement objective is then to determine $\underline{S}_{10}(\underline{K})$, or its value in certain important directions such as the on-axis value $\underline{S}_{10}(0)$.

\subsection{Generalization of the Three-Antenna Measurement Technique}

A three-antenna measurement to determine the gain of three antennas has been available for some time [16]. This involves measuring the ratio of the power input to the transmitting antenna to the available power from the receiving antenna and the separation distance between the antennas for the three combination pairs. The three resulting equations may then be solved for the gain of each antenna.

This approach implicitly assumes that far-field, plane-wave conditions exist and that the three antennas have either known or identical polarization characteristics.

Since only power ratios are measured in the above technique, no information is obtained about the polarization states of the three antennas. Various other methods have been used to measure antenna polarization [17], but they all involve the use of at least one antenna whose polarization is assumed to be known.

The generalized three-antenna method [12] to be described here does not require a quantitative knowledge of the polarization of any of the antennas. It is sufficient to know that two are approximately linearly polarized and the 
polarization of the third is either linear or circular with an approximate direction of polarization. With this knowledge and the measurement of the appropriate quantities, both the gain and polarization of one or more of the antennas can be determined.

Kerns [1] derives the coupling equations for the general situation when only one of the three antennas is reciprocal. For simplicity, and because it is the usual case, we will assume that the two antennas which are used in the receiving mode are reciprocal.

Now suppose that we have two antennas whose transmission characteristics are given by $\underline{S}_{10}(\underline{K})$ and $\underline{S}_{10}(\underline{K})$ when each antenna is placed in a specific orientation in the reference coordinate system. The prescribed orientation is such that the antenna coordinate system is coincident with the reference system. The antenna coordinate system is defined with its z-axis in the electrical boresight direction, and the $x$ - and $y$-axes defined by reference lines scribed on or otherwise defined on the antenna structure. The placement of these fiducial marks on the antenna is essentially arbitrary, but once chosen they fix the axes of the antenna and therefore the coordinate system used to define $\underline{S}_{10}(\underline{K})$. In practice, it simplifies and improves the accuracy of the measurement to place the reference lines so that one axis is approximately parallel to the principal component of the electric field. The $y$-axis will be so chosen in the following.

With these choices of references axes, let antenna number 1 be placed in the prescribed orientation in the reference coordinate system and used as the transmitting antenna. Let the other antenna be placed in a receiving orientation by a 180 deg rotation in the reference coordinate system about the $y$-axis and a translation along the $z$-axis to $z=d$. The two antennas are shown schematically in figure 3 with appropriate terminal surfaces and wave amplitudes shown.

In the following development, the transmission charateristic $\underline{S}_{10}(\underline{K})$ will be used, but since the objective is to determine gain and polarization in the boresight direction, we need only the on-axis value, $\underline{S}_{10}(0)$. A simplified notation may therefore be adopted which will eliminate the double subscript and the explicit reference to $\underline{K}=0$. Both linear and circular components of 


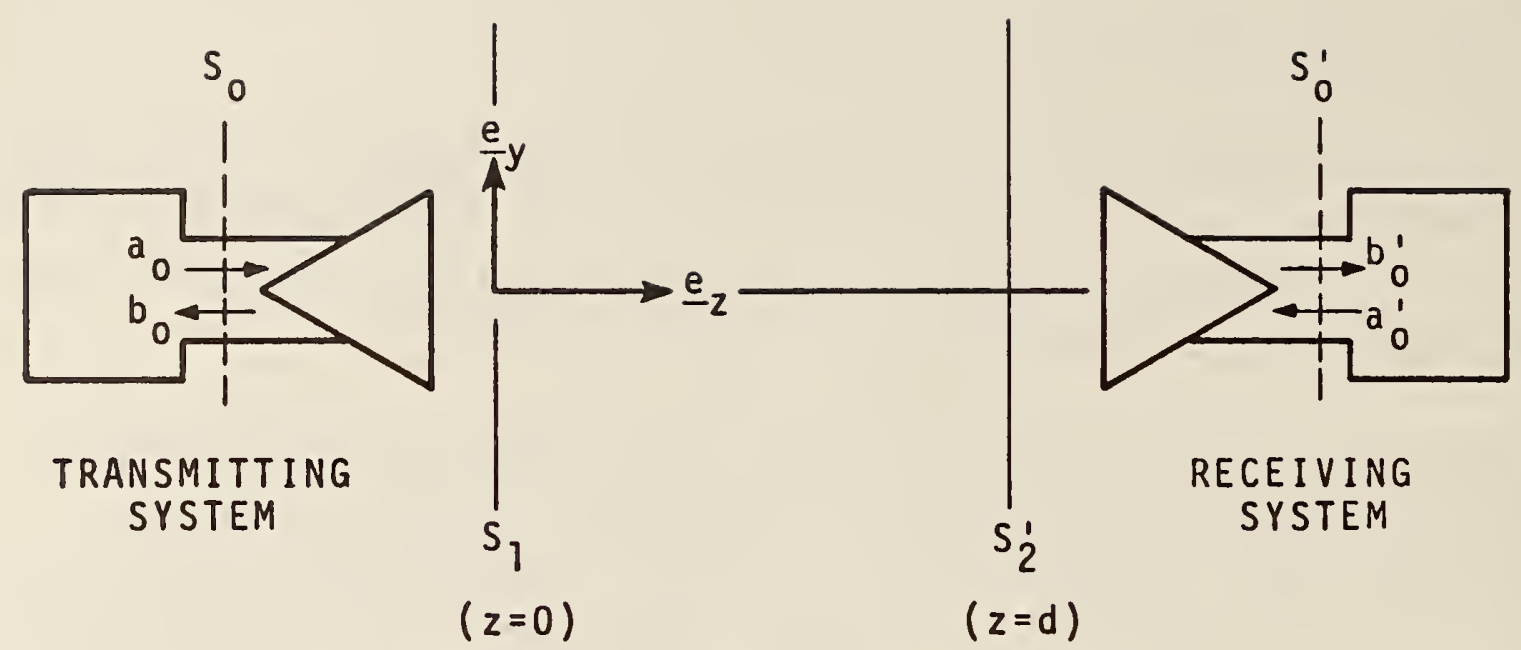

Figure 3. Schematic of two antennas oriented for measurement.

$\underline{S}_{10}(0)$ will be required, and these are defined as follows. Let $\underline{e}_{x}$ and $\underline{e}_{y}$ be unit basis vectors in the $x$ and $y$ directions and define [18]

$$
\begin{aligned}
& \underline{e}^{+}=\left(\underline{e}_{x}+i \underline{e}_{-y}\right) / \sqrt{2}, \\
& \underline{e}^{-}=\left(\underline{e}_{x}-i \underline{e}_{-y}\right) / \sqrt{2} .
\end{aligned}
$$

Then

$$
\underline{E}_{R}=\underline{e}^{+} \exp [i(k z-\omega t)]
$$

and

$$
\underline{E}_{-L}=\underline{e}^{-} \exp [i(k z-\omega t)]
$$

are respectively right and left circularly polarized plane waves traveling in the $+z$ directions. The time dependence here is $\exp (-i \omega t)$, and the definitions of polarization used here are in agreement with the IEEE Standard No. 149 (Test Procedure for Antennas, 1979), and correspond to the definitions of right- and left-handed screw threads. 
The linear and circular components of $\underline{S}_{10}(0)$, using the simplified notation, will be referred, to as $X, Y, R$ and $L$ such that $\underline{S}_{10}(0)=X e_{-x}+Y e_{-y}$ $=\mathrm{Re}^{+}+\mathrm{Le}^{-}$, and the transformation equations are

$$
\begin{array}{ll}
R=(X-i Y) / \sqrt{2}, & L=(X+i Y) / \sqrt{2}, \\
X=(R+L) / \sqrt{2}, & Y=i(R-L) / \sqrt{2} .
\end{array}
$$

The on-axis components for the three antennas will be distinguished by a single subscript, $S_{10 x}^{1}(0)=X_{1}, S_{10 y}^{2}(0)=Y_{2}$, etc.

In the boresight direction, the gain and polarization quantities are given by

$$
\begin{aligned}
& \text { Gain }=G=\frac{4 \pi k^{2}\left(|X|^{2}+|Y|^{2}\right)}{\left(1-\left|S_{00}\right|^{2}\right)}=\frac{4 \pi k^{2}\left(|L|^{2}+|R|^{2}\right)}{\left(1-\left|S_{00}\right|^{2}\right)}, \\
& \text { Linear Polarization Ratio }=\rho_{\ell}=\frac{X}{Y}, \\
& \text { Circular Polarization Ratio }=\rho_{C}=\frac{L}{R}, \\
& \text { Axial Ratio }=A=\frac{R|+| L \mid}{|R|-|L|} \text {, and } \\
& \text { Tilt Angle }=\tau=1 / 2 \arg \left(\rho_{C}\right) .
\end{aligned}
$$

With antennas 1 and 2 in their initial orientation as previously described and shown in figure 3 , the coupling equation is $[1,13,15]$

$$
X_{1} X_{2}-Y_{1} Y_{2}=\lim _{d \rightarrow \infty}\left(\frac{b_{0}^{\prime}(d) d\left(1-\Gamma_{2} \Gamma_{2}\right) e^{-i k d}}{2 \pi i k}\right) \equiv D_{12}^{i},
$$

where $\Gamma_{2}$ is the reflection coefficient for antenna number 2 , and $\Gamma_{\ell}$ is the reflection coefficient for the load.

Now let the receiving antenna be further reoriented by rotating it about the $z$-axis by $90 \mathrm{deg}$ in the direction from $x$ to $y$. The coupling equation for this orientation is 


$$
X_{1} Y_{2}+X_{2} Y_{1}=\lim _{d \rightarrow \infty}\left(\frac{b_{0}^{\prime \prime}(d) d\left(1-\Gamma_{\ell} \Gamma_{2}\right) e^{-i k d}}{2 \pi i k}\right) \equiv D_{12}^{\prime \prime},
$$

where $b_{0}^{\prime \prime}(d)$ denotes the output wave amplitude for the second orientation.

Equations similar to (19) and (20) may be written for the other two antenna pair combinations which yields six equations in six unknown quantities. These six equations may be expressed by the two general equations for transmission from antenna $m$ to antenna $n$ as

$$
\begin{aligned}
& x_{n} X_{m}-y_{n} Y_{m}=D_{n m}^{\prime}, \\
& x_{n} Y_{m}+x_{m} Y_{n}=D_{n m}^{\prime \prime} .
\end{aligned}
$$

These coupling equations have a very simple form and yet contain a great deal of information about the antenna interaction. Since they are written in terms of complex vector components, they take into account the vector character of the field and the receiving antennas response to that vector field. This is in contrast to the Friis transmission equation which relates only the scalar quantities of power and gain and includes no information about the vector nature of the transfer functions. It is possible to write equations using gain, axial ratio, and tilt angle to relate transmitted and received power [18], but such expressions are much more complex and yet contain no more information than eq (21).

To solve the resulting set of six equations we define

$$
\Delta_{n m}=\left(D_{n m}^{\prime}-i D_{n m}^{\prime \prime}\right) / 2 \text {, }
$$

and

$$
\sum_{n m}=\left(D_{n m}^{\prime}+i D_{n m}^{\prime \prime}\right) / 2
$$

and note that eq (21) can be written as

$$
R_{n} R_{m}=\Delta_{n m}, \quad L_{n} L_{m}=\sum_{n m} \text {. }
$$


The solution for circular and linear components now follows and gives, for example,

$$
\begin{aligned}
& R_{1}=+\sqrt{\frac{\Delta_{21} \Delta_{13}}{\Delta_{32}}}, \\
& L_{1}= \pm \sqrt{\frac{\Sigma_{21} \Sigma_{13}}{\Sigma_{32}}},
\end{aligned}
$$

and

$$
\begin{aligned}
& x_{1}= \pm \frac{1}{\sqrt{2}}\left(\sqrt{\frac{\Delta_{21} \Delta_{13}}{\Delta_{32}}} \pm \sqrt{\frac{\Sigma_{21} \Sigma_{13}}{\Sigma_{32}}}\right) \\
& Y_{1}= \pm \frac{i}{\sqrt{2}}\left(\sqrt{\frac{\Delta_{21} \Delta_{13}}{\Delta_{32}}} \mp \sqrt{\frac{\Sigma_{21} \Sigma_{13}}{\Sigma_{32}}}\right) .
\end{aligned}
$$

In these equations the signs are correlated vertically but not horizontally. If the upper signs are used in one of the equations, they must be used in the other two also. A qualitative knowledge of $\left|\rho_{\ell}\right|$, either from a priori information or the measured data, is required to indicate the proper choice. One choice will yield $\left|\rho_{\ell}\right|>1$ while the other choice gives $\left|\rho_{\ell}\right|<1$, and the proper choice will yield results consistent with $\left|\rho_{\ell}\right|$.

Difficulties can arise in the solution of eq (24) if one or more of the antennas is nearly circularly polarized. For example, $\Delta_{32}$ may be nearly zero if antenna 3 has nearly left-circular polarization. The two practical antenna combinations are therefore either three linearly polarized antennas or two linearly and one circularly polarized antenna. In the first case the gains and polarizations are determined for all three, while in the latter the characteristics of only the circular antenna will be completely determined.

If, however, three circularly polarized antennas are nominally polarization matched, then we can determine the principal components from either eq (24a) or (24b). The circular polarizations can then be determined by an improved polarization measurement technique which is discussed in detail in Section 7. Similarly, if three linearly polarized antennas are nominally polarization matched, then we can determine the principal components from 
either eq (25a) or (25b). Then, the linear polarizations can also be determined using the improved polarization measurement technique. Although in practice, these improved procedures are generally the rule, it is necessary to understand thoroughly the above notations and development to facilitate the comprehension of later sections.

\subsection{Measurement Methods for the Extrapolation Technique}

The data required to determine the $D_{n m}$ 's are the ratio of output to input signals for the two antenna orientations, $\left|b_{0}^{\prime}(d) / a_{0}\right|$ and $\left|b_{0}^{\prime \prime}(d) / a_{0}\right|$, and the phase change resulting from the second antenna rotation, $\arg \left(b_{0}^{\prime \prime}(d) / b_{0}^{\prime}(d)\right)$. This information, plus the separation distance $d$, the frequency $f$, and the necessary reflection coefficients determine the $D_{n m}$ 's; the transmission components can then be found from eqs (19) through (25). In principle the required data could be obtained by well known far-field measurement methods, but the extrapolation measurement approach will be described here because it has definite advantages for accurate absolute gain and polarization measurements.

The theory of the extrapolation technique was developed by wacker [11], and the main result is that for any two essentially arbitrary antennas the received signal as a function of separation distance can be accurately represented by the power series

$$
b_{0}^{\prime}(d)=\frac{a_{0}}{1-\Gamma_{n} \Gamma_{\ell}} \sum_{p=0}^{\infty} \frac{\exp [i(2 p+1) k d]}{(d)^{2 p+1}} \sum_{q=0}^{\infty} \frac{A_{p q}}{d^{q}} .
$$

The significance of each term and subseries may be seen more clearly if a few terms are written out explicitly to give

$$
\begin{aligned}
b_{0}^{\prime}(d) & =\frac{a_{0}}{1-\Gamma_{n^{\Gamma} \ell}}\left\{\frac{e^{i k d}}{d}\left(A_{00}+\frac{A_{01}}{d}+\frac{A_{02}}{d^{2}}+\frac{A_{03}}{d^{3}}+\cdots\right)\right. \\
& \left.+\frac{e^{3 i k d}}{d^{3}}\left(A_{10}+\frac{A_{11}}{d}+\frac{A_{12}}{d^{2}}+\cdots\right)+\frac{e^{5 i k d}}{d^{5}}\left(A_{20}+\cdots\right)+\cdots\right\} .
\end{aligned}
$$

The first series represents the direct transmission signal including near-zone terms, the second series the first-order multiple reflection between the two 
antennas, and the third series the higher order multiple reflections. Equations (19) and eq (27) show that by first neglecting the multiple reflection and then higher order terms in 1/d (assume far-field conditions) in eq (27), we can obtain the relationship

$$
D_{n m}^{\prime}=\lim _{d \rightarrow \infty} \frac{b_{0}^{\prime}(d) d \quad\left(1-\Gamma_{\ell} \Gamma_{n}\right)}{2 \pi i k} e^{-i k d}=\frac{A_{00}}{2 \pi i k} .
$$

Hence, $A_{00}$ becomes the measurement objective.

In conventional far-field measurements, $d$ is made large enough that the only significant term in eq (27) is the one involving $A_{00}$. This requires very long ranges for some antennas, and as the distance is increased, the error due to ground reflections increases. In the extrapolation technique, measurements are made over a range of distances, and the data fitted to a series like eq (26). When all significant terms have been evaluated, $A_{00}$ will be given more accurately than if the higher order terms had been neglected. Another advantage is that the proximity correction at any distance equal to or greater than the measurement distances may be accurately calculated, which makes accurate measurements possible at reduced range distances. A means for correcting for multipath effects is also included.

\subsection{Description of Measurement System}

The five subsystems which make up the total measurement system are the extrapolation range, a stable transmitting signal source, the receiving system, a data acquisition system, and a computer (fig. 4). The particular subsystems described here are not necessarily the only ones which will meet the required specifications.

The extrapolation range (fig. 4) consists of two towers and a set of accurately aligned rails on which one of the towers moves. The rails on the NBS range were initially aligned using a transit, precision level, and laser straightness interferometer so that the maximum angular deviation was less than $\pm 0.02^{\circ}$ about any axis and the maximum linear deviation was $\pm 0.1 \mathrm{~mm}$ (see fig. 5). During subsequent years, the rails have required minor readjustments to maintain that tolerance. 


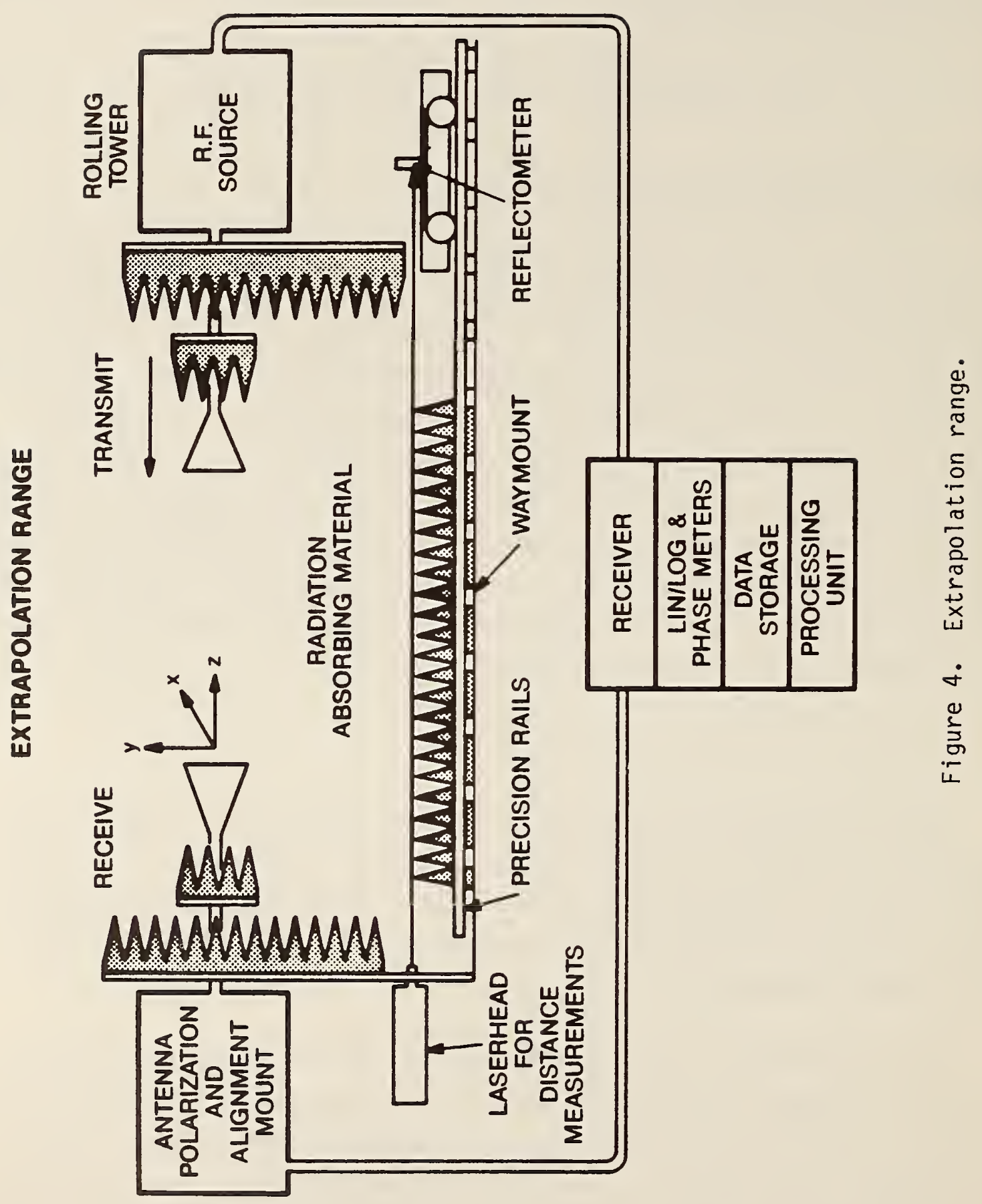




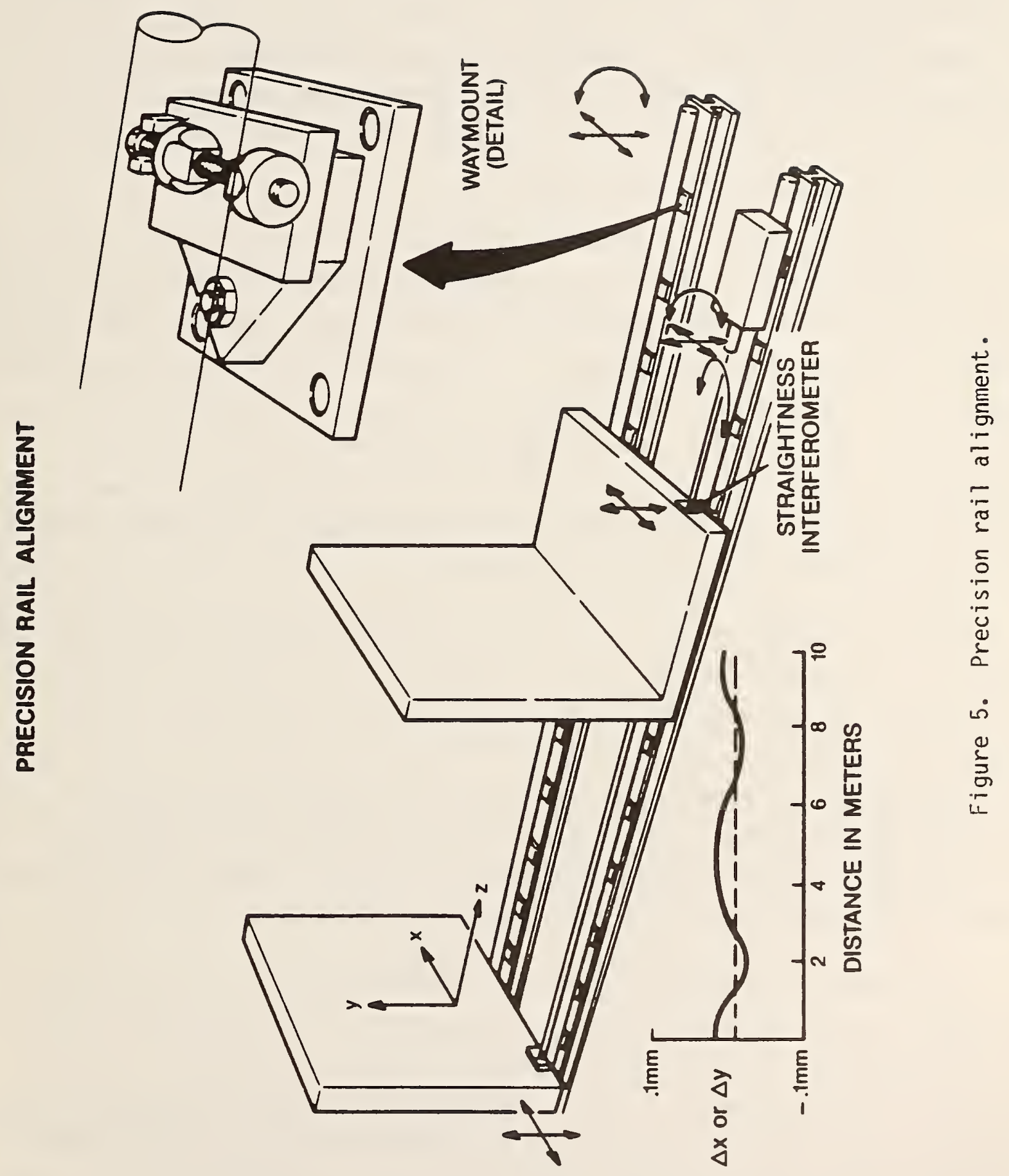


The moving tower is supported by roller bearings so that the tower can be moved over the distance of the range as accurately as the rails are aligned. A variable speed drive system is used to move the cart uniformly during the extrapolation measurement. Plus and minus $z$ movement must be smooth. The variable speed is used primarily so that approximately 10 to 20 data points per wavelength are taken over a range where the insertion loss changes from 0 to $-20 \mathrm{~dB}$. Thus, the time for the tower's $z$-axis movement varies depending on the frequency and the total distance covered.

The separation distance, which must be measured to an accuracy of about 0.1 percent over the total range, can be determined by measuring the voltage across a precision multiturn potentiometer whose rotation angle is proportional to the separation distance between the towers. The important requirement is that the readout be a linear function of distance. The present system uses a laser measurement system which accurately measures the distance over the $10 \mathrm{~m}$ range. Although data are not usually recorded over the complete range, the towers containing the antennas must be brought close enough together to allow the generator and load ports to be connected together.

The towers support parts of the source, receiver and data systems, and the rotators for initially positioning the antennas and for polarization measurements. Means are provided for accurately aligning the two antennas and maintaining that alignment as the separation distance is varied from $0 \mathrm{~m}$ to about 2 $a^{2} / \lambda$, where $a$ is the largest aperture size of the antennas being measured. The required alignment accuracy is a function of the antennas being measured and should be such that the maximum misalignment will cause a change in the received signal of less than $0.01 \mathrm{~dB}$ at the farthest distance.

The antennas are mounted in specially designed gimbals which provide the required rotations about all three axes necessary to align the antennas (fig. 6 ) and to perfonn the required reorientations during the measurements. An important part of the measurement scheme is mounting the transmitting and receiving antennas. The antennas must be mounted on alignment gimbals such that there is sufficient support to eliminate sag and azimuth variations. Depending on where the point of connection is made, whether it is at the waveguide flange or some sort of coaxial connector (Type-N, APC-7, SMA), we must ensure that the antenna does not lose its alignment during any period of the measurement process. 


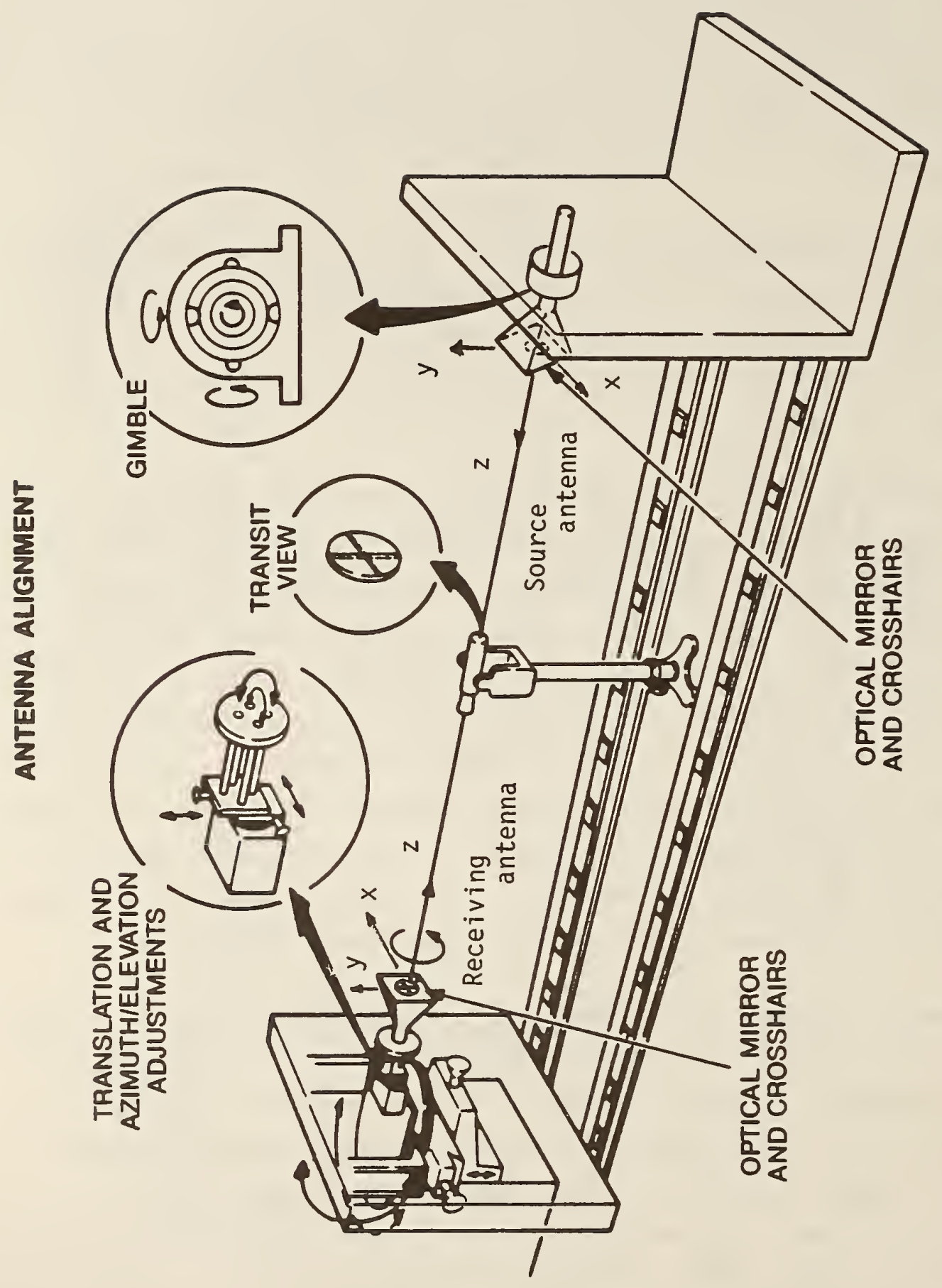

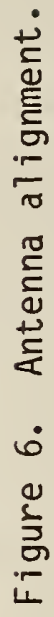


A synthesized signal source with associated equipment used in fixed frequency measurements is shown schematically in figure 7 . The signal source provides a c.w. signal which is stable in both frequency and power during measurements. Amplitude stability of $\pm 0.01 \mathrm{~dB}$ over a 5 to 10 min period is desirable, but a uniform drift of as much as $0.05 \mathrm{~dB}$ during this period can be allowed. Some of the drift can be averaged out by making measurements in both + and $-z$ directions. The frequency stability requirement is primarily to reduce phase drift.

Leakage from the synthesizer, waveguide joints, or other components can cause very serious errors in any antenna measurement and care must be exercised to reduce all such leaks to insignificant levels. (As an example, for the effect of the leaks to be less than $0.01 \mathrm{~dB}$, we can require that the leaks be less than $65 \mathrm{~dB}$ below the direct signal.)

A block diagram of the receiving system is al so shown in figure 7 . This system must measure the received signal amplitude over a dynamic range of about $50 \mathrm{~dB}$ as well as the phase difference between the received signal and a reference signal. The large dynamic range is required since the received signal will be large when generator and load are connected directly together for the measurement of $a_{0}$ and will be very small when the receiving antenna is rotated by $90 \mathrm{deg}$ to measure the cross component of the field. The actual dynamic range which the receiver sees can be reduced significantly by measuring part of the signal change with a precision attenuator. For example, it was only necessary to calibrate the receiver of figure 7 over a $20 \mathrm{~dB}$ range. The attenuator is also used to calibrate the receiver response.

The phase is measured with respect to a reference, $\phi_{r}$, which is independent of the orientation of the receiving test antenna. $\phi_{r}$ may vary as the separation distance is changed as long it has the same variation for both orientations of the test antenna. One practical approach on large outdoor extrapolation ranges is to place a small antenna to the side of the test antenna. This reference antenna should be far enough away to eliminate interaction and still close enough to receive a significant signal. A small standard gain horn or open-ended waveguide is usually adequate. On indoor or small outdoor ranges, the receiver can be phase-locked to a reference signal obtained from the source via a cable. 


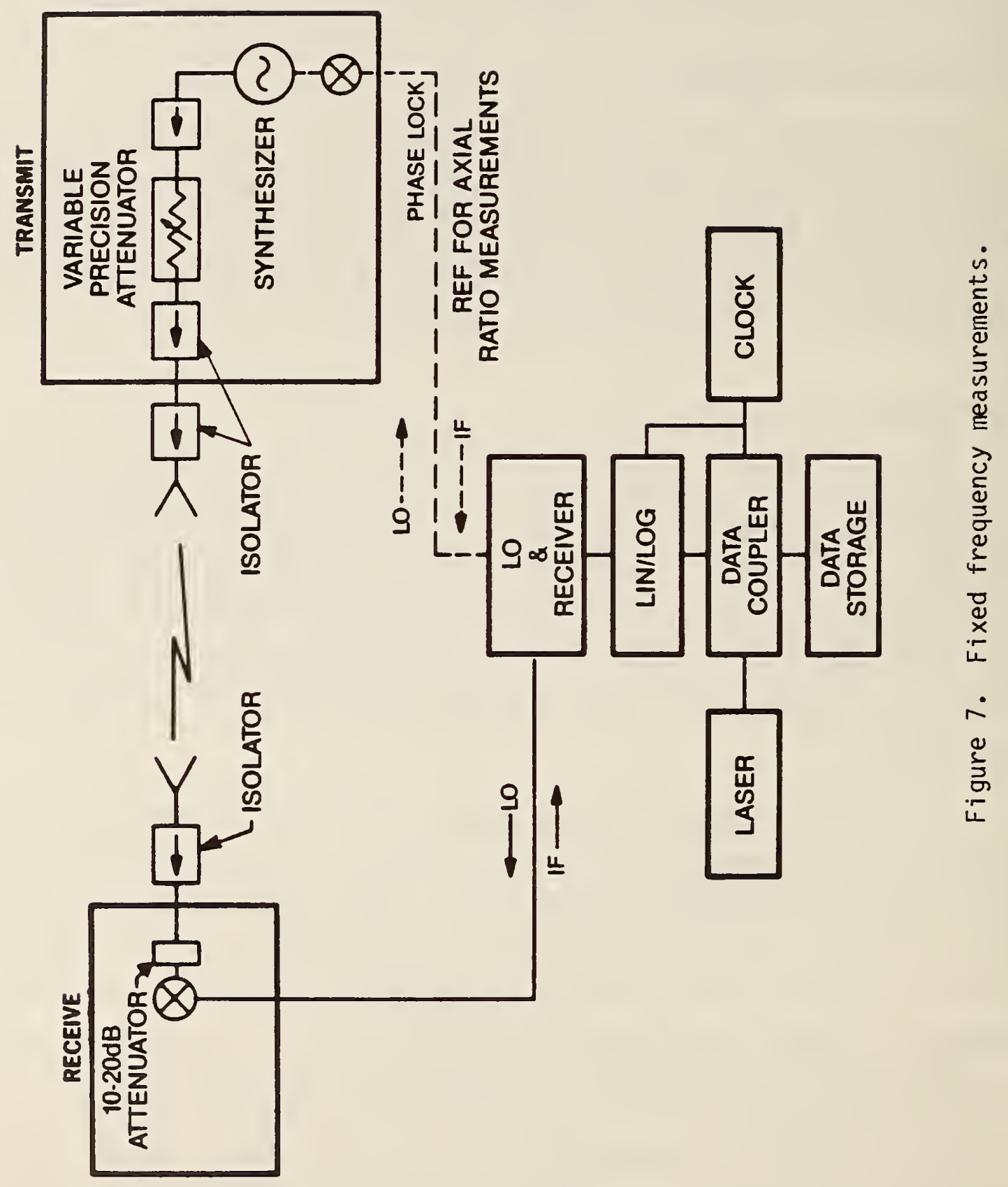


The receiver outputs are fed into the data system, which may be as simple as an $x-y$ recorder that plots the amplitude and phase as a function of the separation distance. However, the requirements of accuracy, speed, and economy usually demand a digital system that records the data on magnetic tape or disk in a format suitable for computer processing.

\section{Measurement Procedures}

Before the actual work on the range begins, the input reflection coefficients for each antenna and for the generator and load ports are measured so that corrections for mismatch errors can be made. This is very important when one is attempting high accuracy measurements. A tuned reflectometer [19] or network analyzer is normally used for this purpose.

\subsection{Al ignment of Antennas}

Currently, there are two prescribed methods for aligning antennas. One depends on the mechanical properties of the antenna while the other depends on its electrical properties. Both methods will be described here. The goal of the alignment procedure is to align the mechanical or electrical boresights of the transmitting and receiving antennas with the reference $z$-axis of the extrapolation range (fig. 5) and to provide a reference for the polarization measurements.

Let us define three coordinate systems, one fixed to each of two antennas and one fixed in space and referenced to the test range. Each antenna system is defined with the boresight direction along the z-axis and the y-axis nominally along the major axis of the polarization ellipse. The exact location of the axes is not critical but they must be clearly defined (fig. 5). The reference, or space, coordinate system has its $x$-axis horizontal, the y-axis vertical, and the $z$-axis along the center of the test range (fig. 6).

For the mechanical alignment method, the first step in the measurement is to place the antennas in the prescribed orientations with respect to the reference coordinate system. This is accomplished by first rotating the antennas about their $z$-axes until their $x$-axes reference marks are horizontal. This is easily done by viewing the antennas with a leveled transit placed 
between the antennas. The transit is then aligned to determine the $z$ direction of the range's rails. This is accomplished by placing fine wires across the $x$ - and $y$-axes of the transmitting horn, moving the cart in and out, and aligning the transit so that there is no change in the position of the cross hairs. A precision flat plate is then attached to the antenna aperture and a precision mirror is centered on the plate. By using an autocollimator on the transit, we then precisely align the antenna so that its z-axis is parallel to the rails. The transit is then rotated about its y-axis precisely $180 \mathrm{deg}$, and, in a similar manner, the receiving horn is aligned. An additional check should be taken especially when the antennas are being aligned for polarization measurements, that is, when the antenna is rotated 360 deg about its z-axis, the mirror must remain collimated and the intersection of the cross hairs must remain constant.

In the electrical alignment method, first the x-axes of the antennas are leveled with the transit just as in the former method. Now, however, the towers are set for maximum separation, and the two antennas are alternately rotated about their $x$ - and $y$-axes to achieve maximum signal transfer. When this is achieved the boresight directions for both antennas are coincident with the reference $z$-axis, and the first stage of the alignment has been completed. The next step is to adjust the receiving antenna relative to its gimbal mount so that the z-axis of the mount is coincident with the boresight direction of the antenna. This condition is achieved by rotating the antenna $180 \mathrm{deg}$ about the mount axis, noting the shift in boresight direction, and adjusting the antenna relative to the mount until a 180 deg rotation leaves the boresight direction unchanged. Several iterations may be required.

\subsection{Receiver Calibration and Determination of $a_{0}$}

When the alignment has been completed, the towers are moved close together so that the generator and load ports may be connected together. The calibrated variable precision attenuator is then used to determine the linear range of the mixer, calibrate the receiver response, and determine $a_{0}$, the input to the transmitter. The linear response range of the mixer is determined by measuring the change in output signal, $\Delta B$, resulting from a change in the input precision attenuator, $\Delta \mathrm{L}_{1}$, for various signal power levels. When operating at the correct power level, the difference (in decibels) between $\Delta B$ and 
$\Delta \mathrm{L}$, will be a constant which is independent of the received power. During the rest of the measurements the signal power level is maintained within the linear region.

The receiver is then calibrated by systematically varying $L_{1}$ over a 0 to $20 \mathrm{~dB}$ range and recording the corresponding receiver output values. The resulting calibration curve is used to correct the measured data.

Several steps are required in order to obtain the desired quantity, $\left|b_{0}^{\prime}(d) / a_{0}\right|$, in terms of the receiver output, $B(d)$, the change in the input attenuator for the generator-load hookup, $\Delta L_{1}$, and certain mismatch factors. The output amplitude meter measures relative signal changes, $\left|b_{0}^{\prime} / b_{r}\right|$, on a logarithmic scale and the observed receiver output, $B^{\prime}$, is then given by

$$
B^{\prime}(d)=20 \log \left|\frac{b_{0}^{\prime}(d)}{b_{r}}\right| \text {. }
$$

The reference amplitude, $b_{r}$, is essentially arbitrary but must remain constant during the measurement. With the generator and load connected together, the input attenuator is set on $L_{j}$ and the receiver gain set for $B^{\prime}=0$. The reference amplitude is then given by [20]

$$
b_{r}=\frac{b_{g}}{\left(1-\Gamma_{g}{ }^{\Gamma} \ell\right.}
$$

where $b_{g}$ is the wave amplitude that the generator would deliver to a matched load. The antennas are now replaced with antenna $m$ connected to the generator and antenna $n$ connected to the load. The input attenuator is reset to a new value, $L_{f}$, which results in a new $b_{g}$ given by $b_{g}^{\prime}=a b_{g}$. If $\Delta L_{1} \equiv L_{j}-L_{f}$, then $20 \log |\alpha|=\Delta L_{1}$. The input amplitude to antenna $m$ is

$$
a_{0}=\frac{b_{g}^{\prime}}{\left(1-\Gamma_{m} \Gamma_{g}\right)}
$$

and from eqs (29), (30), and (31) we obtain

$$
20 \log \left|\frac{b_{0}^{\prime}(d)}{a_{0}}\right|=B^{\prime}(d)-\Delta L_{1}-20 \log \left|\frac{\left(1-\Gamma_{g}{ }^{\Gamma}\right)}{\left(1-\Gamma_{g} \Gamma_{m}\right.}\right| \text {. }
$$


$\Delta L_{1}$ is chosen so that $B^{\prime}\left(d_{m i n}\right)=0$, where $d_{m i n}$ is the minimum distance at which data is to be taken. The towers are now moved slowly apart and the resulting amplitude $B^{\prime}(d)$ and phase $\phi^{\prime}(d)$ data recorded as a function of distance.

The receiving antenna is then rotated about its $z$-axis by 90 deg, the output attenuator changed by $\Delta \mathrm{L}_{2}$, if necessary, to restore the signal level at the receiver to its previous range, and the amplitude $B^{\prime \prime}(d)$ and phase $\phi^{\prime \prime}(d)$ data again recorded as a function of distance. This measurement sequence is repeated for all three antenna pairs to provide the necessary data for the extrapolation calculations and solution of the three-antenna equations.

The generator and load connections are a very important part in the measurement process and great care must be taken to assure repeatable, accurate measurements in the hookups. For example, an error in the generator-load hookup translates directly into an error in the average gain of the antenna pair being measured. (Typically, generator-load measurements repeat to within $0.02 \mathrm{~dB}$.$) The generator-load connection is necessary to determine absolute$ gain which accounts for the ohmic loss of the antennas. This is in contrast to measuring the directivity of an antenna, estimating the ohmic loss and calculating the absolute gain, a procedure used in some laboratories.

A potential source of error often appears at the junction of the coaxial cable and the coax-to-waveguide adapter. Care must be taken that the connector is neither tightened too tightly, damaging the center pin or too loosely so that the connection can work itself free. A solution to this problem is the use of torque wrenches and the introduction of strain relief loops at the connector joints. Strain relief loops el iminate many problems which occur with movement of the input and output assemblies during generator-load hookups, since the connection is isolated from the general cable movement. Even a single loop securely fastened to the coax-to-waveguide assembly or accompanying waveguide piece provides adequate protection from connector problems (fig. 8).

Another area where problems often occur is the transmission cable itself. Depending on what types of cables are used, e.g. (RG 214-U, RG 9-U, $0.358 \mathrm{~cm}$ ( $0.141 \mathrm{in}$ ) diameter semi-rigid, the following should be verified about the cable: 
(a)

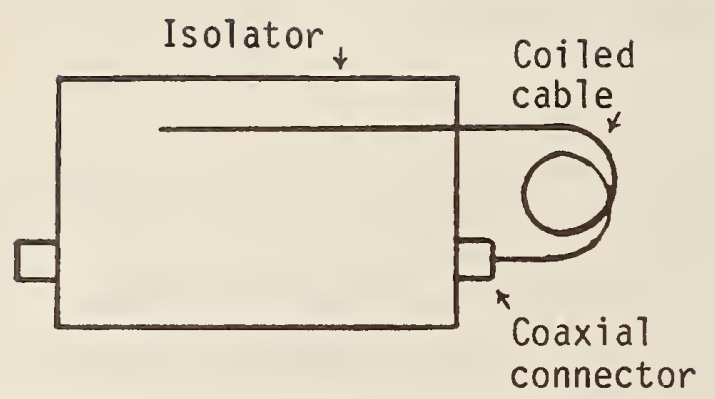

Isolator

(c)

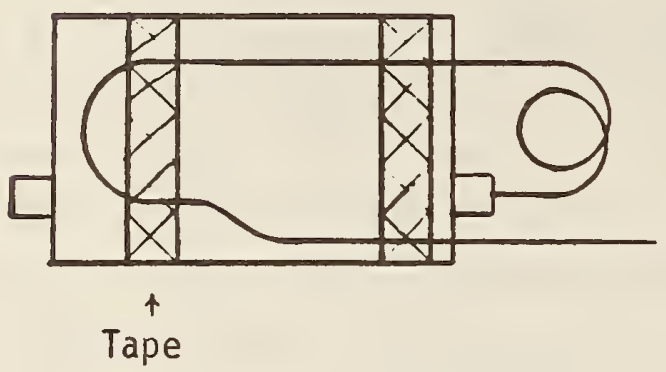

(b)

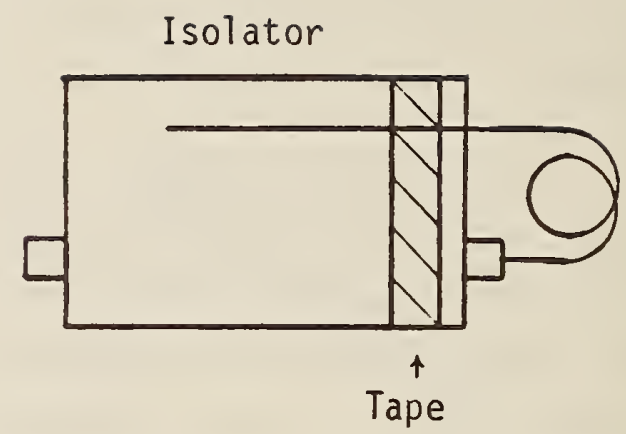

(d)

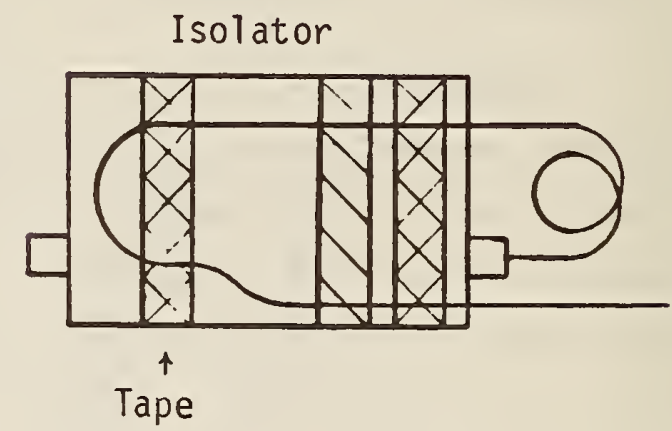

Figure 8. Steps in making a strain relief loop to minimize cable movement at connector joints.

1. It has the appropriate frequency response for the measurement.

2. It has stable (preferably low) loss.

3. It has the durability to withstand occasional bending and movement.

4. It is firmly attached to the connector, primarily to minimize signal leakage.

5. It has durable connectors to insure repeatability.

Depending on the previous usage of the cable, it is always good practice to visually inspect each cable and to test it electrically before installing it in the system.

The assembly of waveguide components can also present problems to the technician where leakage and connection repeatability are concerned. All waveguide joints must be put together in a consistent manner. Care must be taken to minimize leakage by using proper shielding of the joints with copper 
tape or some type of foil. We should also ensure that any waveguide system have sufficient support that the torque on any waveguide component is minimized.

\subsection{Amplitude, Phase and Distance Data, General}

The most apparent application of the extrapolation technique requires fitting complex data (real and imaginary parts of $b_{0}^{\prime} / a_{0}$ ), including the multipath interference, to give all of the coefficients in eq (27). This procedure presents difficult problems in obtaining accurate enough phase and distance data and in processing the large number of data points required. If the phase factor, $\exp [1(2 p+1) k d]$, is to be accurately determined, the distance must be measured to within a small fraction of a wavelength, and many data points will be required to define the periodic multipath interference curve. These problems can rigorously be overcome by first removing the multipath effects from the data and then fitting the square of the amplitude, $\left|b_{0}^{\prime} / a_{0}\right|^{2}$.

$A_{00}$ is then determined more easily, and more accurately, than if complete fitting is done with inadequate phase and distance data. The details of this approach follow.

\subsection{Amplitude Measurements}

The general character of the amplitude data is illustrated in figure 9 for two $X$-band standard gain horns. The effect of the multiple reflections between the antennas is clearly seen in the modulation of the signal with a period of $\lambda / 2$. The relative magnitudes of the various order multiple reflections can be approximated from the peak-to-peak magnitude, $M$, of the modulation curve. For example, if $M \leqslant 1.0 \mathrm{~dB}$, the first-order multiple reflection is at least $25 \mathrm{~dB}$ below the direct signal and the second-order reflection is down by at least $50 \mathrm{~dB}$. For typical data $M$ is usually less than $1 \mathrm{~dB}$ and we may conclude that the effect of the multiple reflections on the measured data will be a small regular modulation that can be removed by fairly simple averaging.

The averaging can be done graphically but it is a very time consuming process and only a few data points will be obtained for the fitting. When the data are recorded digitally, the computer can perform the averaging and derive 


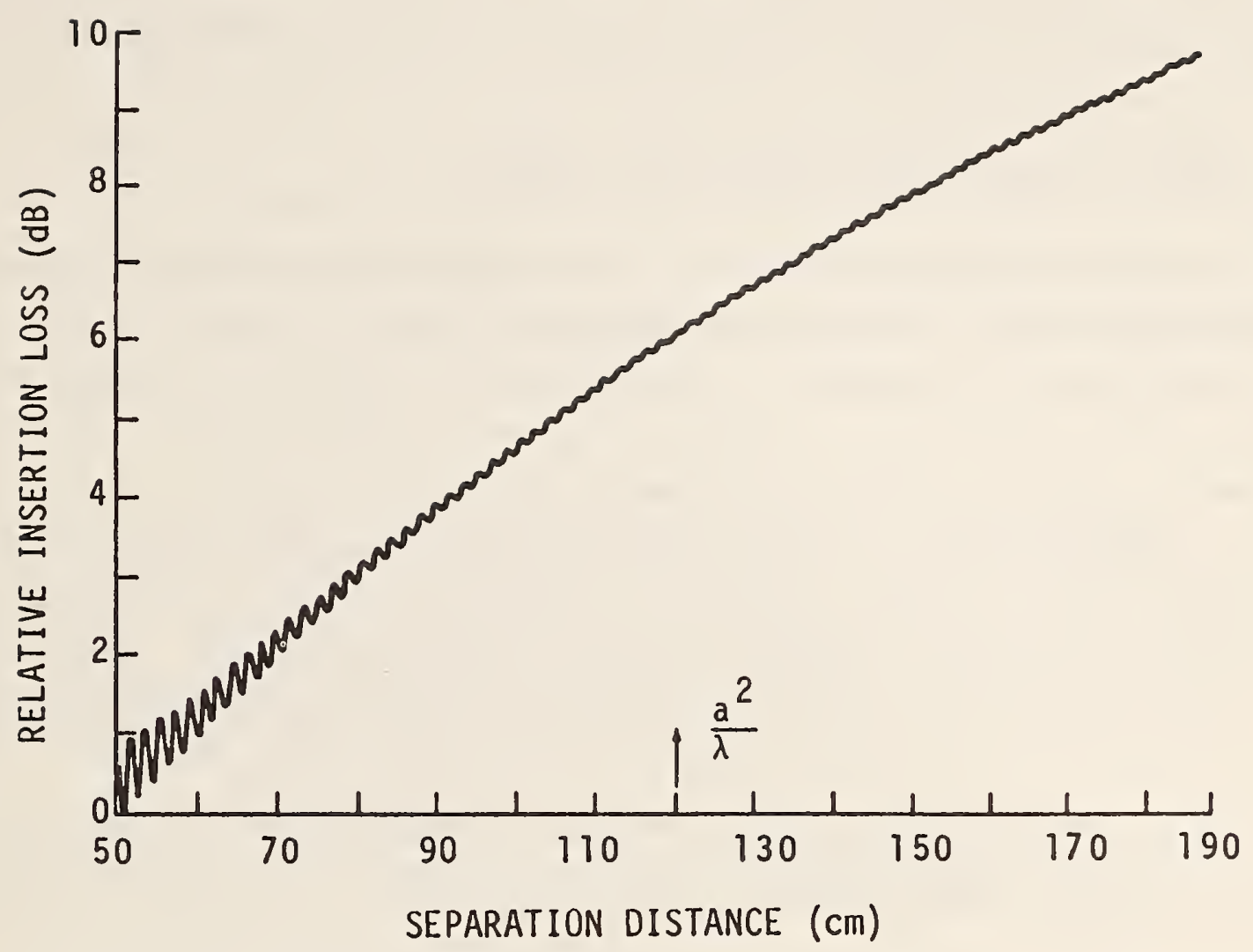

Figure 9. Extrapolation data for two X-band standard gain horns.

many more points in a much shorter time. We usually record 10 to 20 data points over each cycle of the interference pattern, and the computer averages over a fixed number of points or computes a running average. Averaging is typically accomplished over an integral multiple of half wavelengths.

A third alternative is to use a low-pass filter in the output section of the receiver to filter out the modulation produced by the multiple reilections. The modulation frequency can be controlled by the speed of the tower, and is given by $f_{n}=2 V / n \lambda$, where $f_{n}$ is the frequency resulting from the nthorder reflection, $V$ is the velocity of the tower, and $\lambda$ is the wavelength of the $r f$ signal. This procedure works very well at short wavelengths and for phase-locked receivers which have narrow bandwidths. The averaging is then accomplished automatically without any computations. Figure 10 shows the appearance of the modulation at a very slow tower motion and the automatic averaging accomplished at a moderate tower speed. The individual cycles of the multipath are not resolved here because of the short wavelength $(\simeq 5 \mathrm{~mm})$. 


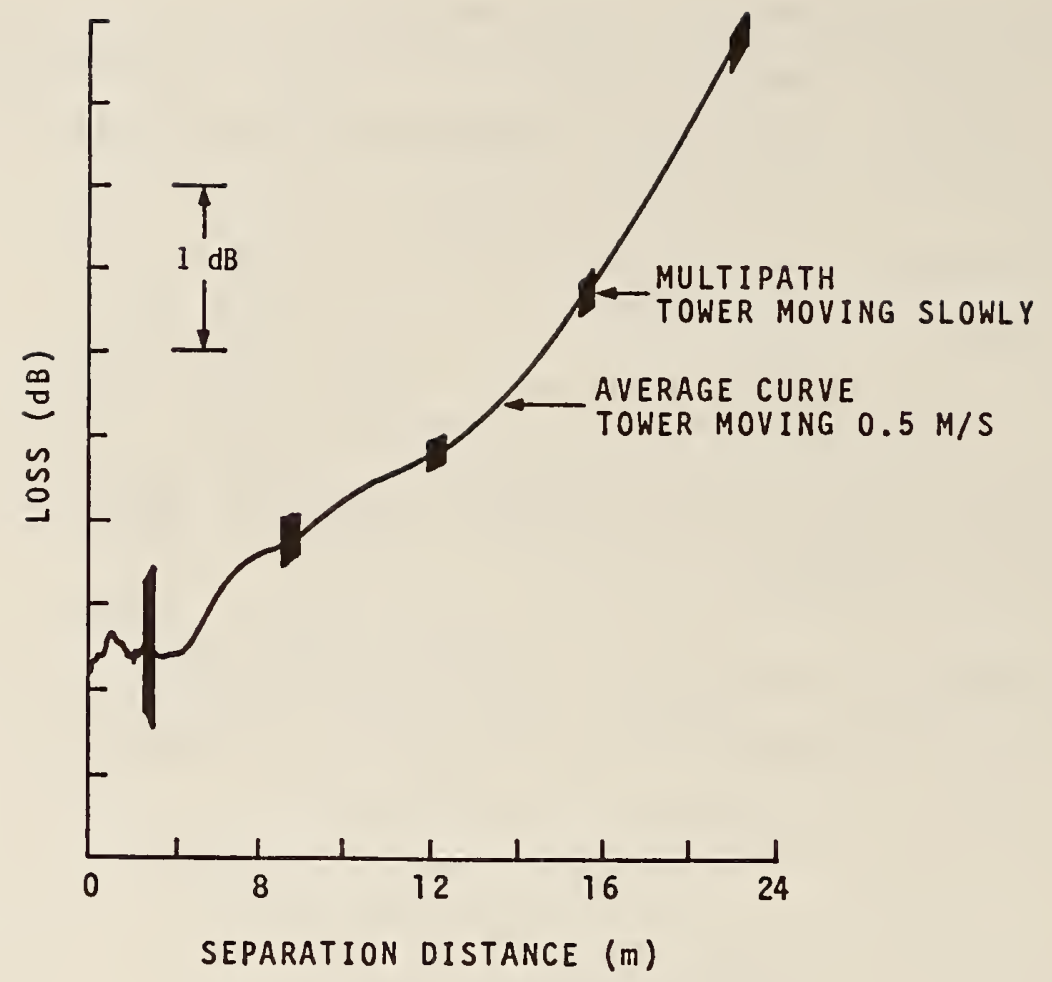

Figure 10. Extrapolation data showing multipath interference and averaged curve for two $45 \mathrm{~cm}$ (18 in), millimeter-wave, parabolic-reflector antennas.

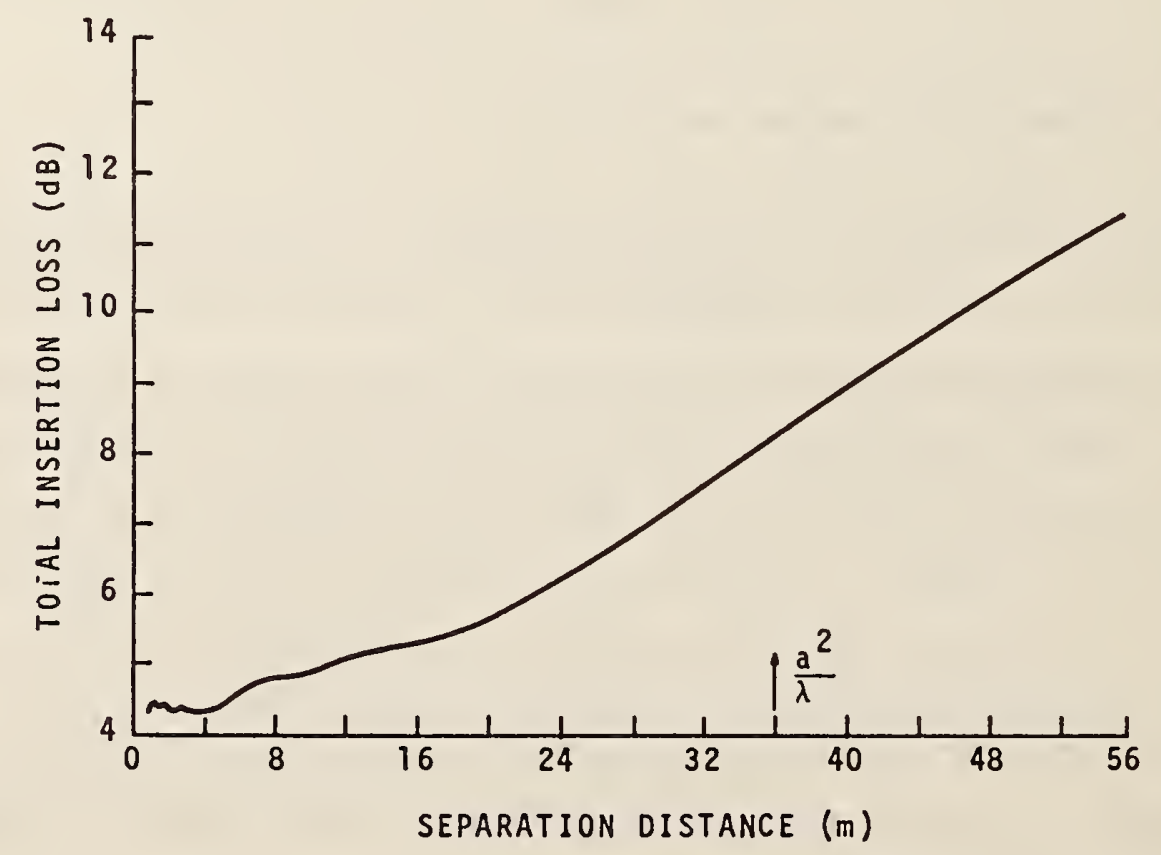

Figure 11. Averaged extrapolation data for the same antenna as in figure 8. 
The result of this process is a number of points on an averaged curve, such as in figure 11. Since all orders of multipath signals have been removed, this received signal can be represented by the first series of eq (27), giving

$$
\frac{b_{0}^{\prime}(d) d\left(1-\Gamma_{n} \Gamma_{\ell}\right) e^{-i k d}}{a_{0}}=A_{00}+\frac{A_{01}}{d}+\frac{A_{02}}{d^{2}}+\frac{A_{03}}{d^{3}}+\cdots \cdot
$$

Since only the amplitude is to be fitted, we take the square of the absolute value of eq (33) and obtain

$$
\left|\frac{b_{0}^{\prime}(d) d}{a_{0}}\right|^{2}\left(\left|1-\Gamma_{n} \Gamma\right|^{2}\right)=A_{00}^{\prime}+\frac{A_{01}^{\prime}}{d}+\frac{A_{02}^{\prime}}{d^{2}}+\frac{A_{03}^{\prime}}{d^{3}}+\cdots \cdot
$$

where

$$
\begin{aligned}
& A_{00}^{\prime}=A_{00} \bar{A}_{00}=\left|A_{00}\right|^{2} \\
& A_{01}^{\prime}=A_{00} \bar{A}_{01}+\bar{A}_{00} A_{01} \\
& A_{02}^{\prime}=A_{00} \bar{A}_{02}+\bar{A}_{00} A_{02}+A_{01} \bar{A}_{01} .
\end{aligned}
$$

(The bar denotes the complex conjugate.)

It is apparent that the coefficient which determines the far-field properties, $A_{00}$, is still determined from the first term in the new series.

The received signal will also be modulated by ground reflections when present. This effect can be distinguished from the multiple reflections by its longer modulation period, $P_{m}$, which also increases with the separation distance. For example, if the ratio of antenna height to separation distance, $h / d$, is $\gg 1, P_{m} \simeq \lambda$; for $h / d \simeq 1, P_{m} \simeq 2 \lambda$; and for $h / d \simeq 1 / 4, P_{m} \simeq 10 \lambda$. If ground reflections are present $h / d$ must be large enough to result in several modulation periods over the measurement distance. This effect can then also be averaged out graphically and by computer, or by simply fitting the data with a low-order series. 
The spacing of the data points is chosen to give between 1000 and 3000 approximately equally spaced points over the measurement interval. We have found it best to record data for repeated runs over the same measurement interval with the tower moving in opposite directions. This tends to randomize amplitude drifts or other short term changes, and is, therefore, preferable to relying on a single run.

The guidelines for choosing a particular distance interval to operate over depend on the types of errors in the data and will be discussed in the section on error analysis. However, at the present stage of development, it appears that the maximum distance, $d_{\max }$, should be at least $a^{2} / \lambda$, but not so great that large ground reflections occur. The minimum distance, $d_{m i n}$, is chosen small enough so that $d_{\max } / d_{\min }>4$, but it should be large enough that three to six coefficients will accurately fit the data [see below eq (41)]. The distance interval which meets these guidelines will depend on the antennas being measured, but will be approximately between $0.2\left(a^{2} / \lambda\right)$ and $2\left(a^{2} / \lambda\right)$. Another criterion is that there be at least $10 \mathrm{~dB}$ total variation in signal level over the distance interval.

\subsection{Phase Measurements}

As stated in the last paragraph in section 2.2, we generally calibrate three antennas that are nominally polarization matched. Using this approach, we can determine to high accuracy the gain of the antennas without need for phase measurements (section 5.2). Axial ratios and tilt angles are then determined using the improved polarization technique described in section 7. Phase measurements have been used to determine gain and polarization parameters, however, and are included here for tutorial purposes and completeness. Phase-versus-distance measurements have been used to reduce the effects of ground reflections when determining gains for broadbeam antennas (appendix A).

Since polarization and gain are independent of any phase factor common to both $X_{n}$ and $Y_{n}$, only the relative phase difference between $D_{n m}^{\prime}$ and $D_{n m}^{\prime \prime}$ need be measured. This is given by [eqs. (19) and (20)]

$$
\Delta \phi_{n m}=\arg \frac{D_{n m}^{\prime \prime}}{D_{n m}^{\prime}}=\lim _{d \rightarrow \infty} \arg \frac{b_{0}^{\prime \prime}(d)}{b_{0}^{\prime}(d)}
$$


and is determined by measuring the phase of $b_{0}^{\prime}(d)$ and $b_{0}^{\prime \prime}(d)$ relative to a common reference, and then computing the phase difference as a function of distance. Some averaging of multipath effects and extrapolation of the phase data also required, and this is very similar to the analysis of the amplitude data.

Figure 12 shows the phase differences for an antenna pair plotted as a function of reciprocal distance, and illustrates the asymptotic character as $d$ becomes large.

4. Numerical Techniques for Antenna Gain and Polarization Measurements

When the measurements have been completed, the data are analyzed to determine $A_{00}^{\prime}$ and the phase difference for each orientation for each antenna pair (sect. 3.5, paragr. 1). The main tasks of the computer processing are to read the data from magnetic tape, fit the data to a power series, and make various plots and tabulations of the measured data and the fitted curves.

The first step in this computer processing is the averaging discussed in a previous section which eliminates the effects of multiple reflections. The amplitude data are then modified to account for the receiver calibration, atmospheric attenuation, impedance mismatch, and the change in the input attenuator $\left[\Delta \mathrm{L}_{1}\right.$ in eq (32)].

The independent and dependent variables, $u$ and $v$, to be used in the fitting are next computed from the amplitude and distance data. $u$ is equal to the reciprocal distance scaled by the factor $a^{2} / \lambda$, and is given by

$$
u=\frac{a^{2} / \lambda}{d}
$$

This scaling is done so that the computer generated plots for any antenna will have a distance scale in units of $a^{2} / \lambda$. It is strictly a matter of convenience in interpreting the graphs and has no effect on the final result.

If the amplitude has been recorded in voltage or decibels, it is converted to a power ratio and multiplied by $d^{2}$ to give the dependent variable $v$. 


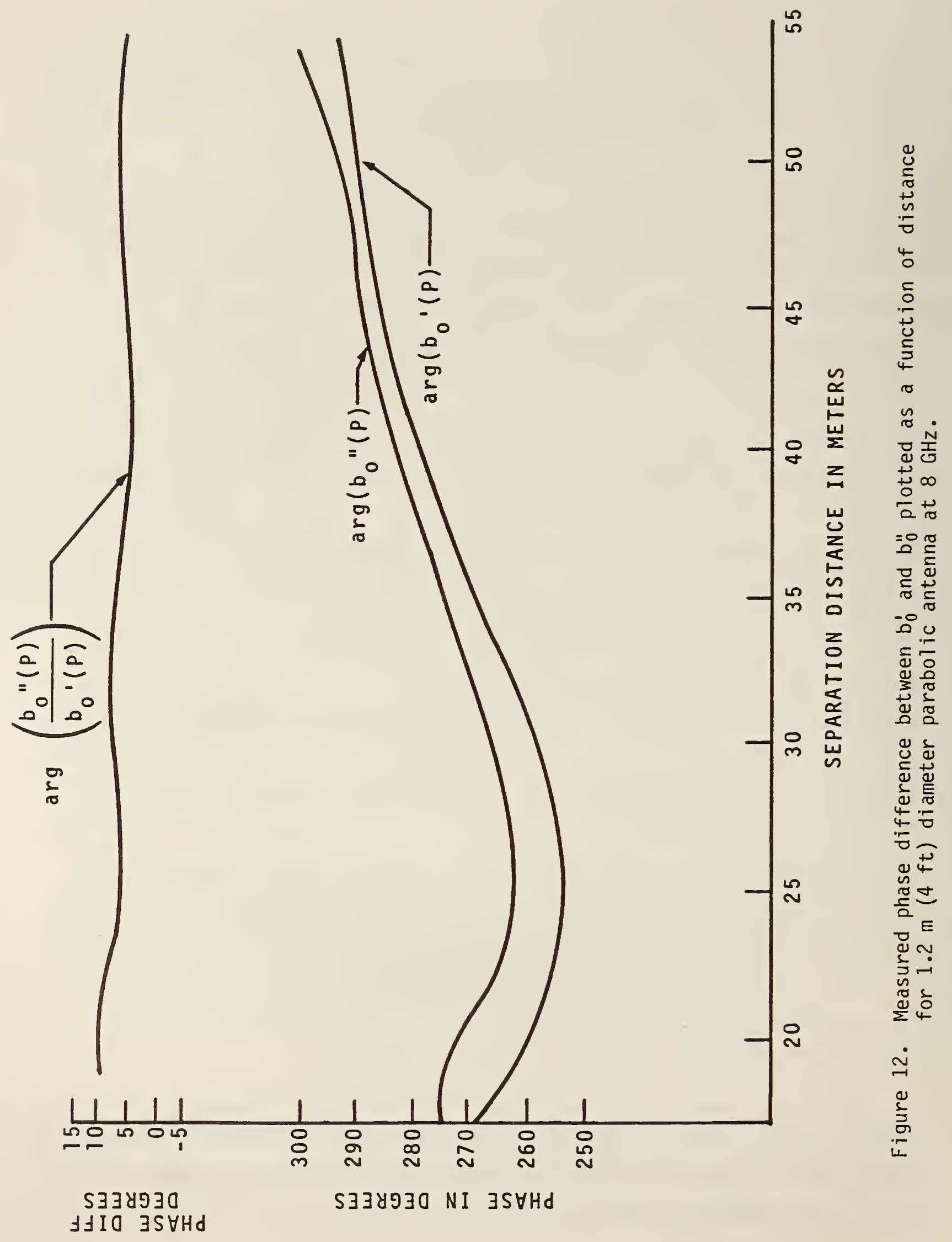




$$
\begin{aligned}
v(u) & =\text { (measured power ratio) } \cdot d^{2} \\
& =\left|\frac{b^{\prime}{ }_{0}(d) d e^{-i k d}\left(1-\Gamma_{n} \Gamma_{\ell}\right)}{a_{0}}\right|^{2}
\end{aligned}
$$

The coefficients of eq (34) for a given number of terms are now determined by fitting $v(u)$ with a series in power of $u$, using the method of least squares. After the coefficients have been determined a graph is produced showing the measured and fitted curves. One such graph is shown in figure 13, which illustrates the general character of these plots. The abscissa is the reciprocal scaled distance, $u$, and therefore increasing distance is in the direction of decreasing abscissa, with $d=\infty$ corresponding to $u=0$. The ordinate is power ratio times the distance squared. Three quantities are shown plotted on the graph as a function of $u$. These are the measured data, the fitted curve, and the difference between the measured and fitted curves. The curve representing the measured data is a plot of eq (37) and therefore includes the effect of measured amplitude, distance, and the atmospheric correction. It is plotted over the range for $u$ for which measurements were taken. If the measurements had been made in the far-field and the distances were measured from the approximate phase centers of the antennas, the curve would have been a horizontal straight line. Its departure from this condition illustrates the near-zone correction as a function of $u$. The fitted curve is plotted over the full range of $u$ and is the evaluation of the polynomial using the derived coefficients.

The difference between the measured and fitted curves is shown in the point plot with one point for each data point. The differences are amplified by a constant factor (given in the captions above the graph) and plotted about the center ordinate so that systematic differences are more apparent. The difference points help detect the presence of small systematic errors and indicate the goodness of the fit.

When only random errors are present, and the correct number of terms are used to fit the data, the difference plot will also be random. When either of these conditions is not fulfilled, the point plot will have a periodic character as is evident in figure 13. 


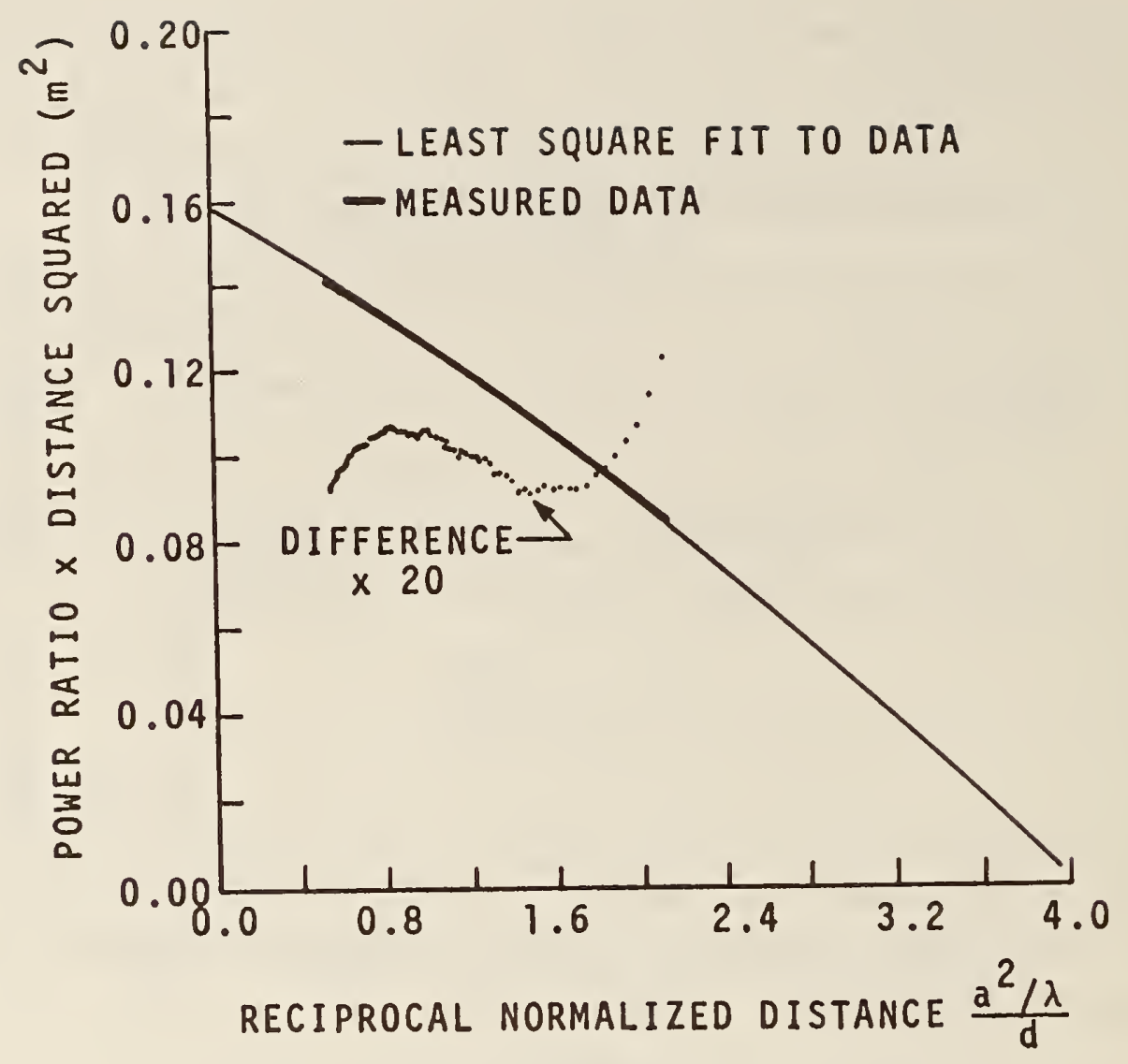

Figure 13. Measured-data, two-term polynomial fit, and residuals for $X$-band conical horn (gain $\approx 22 \mathrm{~dB}$ ).

The correct number of terms to use in the polynomial fit is determined by using the Snedecor-Fisher F-test of statistical significance [21]. To use this test, a set of $\mathrm{N}$ data points is fitted with a sequence of polynomials of increasing order, and the residuals, defined as the sum of the squares of the differences between the measured and fitted curves, are computed for each set of coefficients. If we denote the residuals for the series with $n$ coefficients by $r_{n}$, then the nth coefficient is significant if

$$
F_{n}=\frac{\left(r_{n-1}-r_{n}\right)(N-n)}{r_{n}}>F(1, N) .
$$

Values for $F(1, N)$ can be found in tables of percentiles of the $F$ distribution. For the present case where $N>100, F(1, N)$ is taken to be about 5 . The value of $F_{n}$ is computed for each trial series and printed out with the results and 
if it is greater than 5 , the last term in the series is significant. The series which gives the best significant fit is terminated with the highest order coefficient which is significant, and the first coefficient of that series is $A_{0}^{1}$. Figure 14 shows the graph of the 5 -term series that was determined to be the best fit where all coefficients were significant. There is still a very small $( \pm 0.01 \mathrm{~dB})$ periodic character to the difference plot which is due to residual errors. It is similar in character to ground reflections, but has a longer period, and if a number of cycles are present in the data, it will have a very small effect on the determination of $A_{00}^{\prime}$.

Because only the relative phase between $D_{n m}^{\prime}$ and $D_{n m}^{\prime \prime}$ is required we may arbitrarily assign a 0 deg phase to $D_{n m}^{\prime}$, and we then have

$$
D_{n m}^{\prime}=\frac{\sqrt{A_{00}^{\prime}}}{2 \pi i k}
$$

where $A_{00}^{\prime} \equiv A_{00}$ for notational convenience. From data taken with the same antenna pair after rotation of the receiving antenna about its axis [eq (20)], we obtain a new series and therefore a new constant coefficient denoted by $A_{00}^{\prime \prime} \cdot D_{n m}^{\prime \prime}$ is then given by

$$
D_{n m}^{\prime \prime}=\frac{\sqrt{A_{00}^{\prime \prime}}}{2 \pi i k} e^{i \Delta \phi_{n m}}
$$

The measurement and fitting procedures are carried out for the three antenna pairs which yield the six $D_{n m}$ 's required to solve eqs (22) through (25) and (14) through (18) for the gain and polarization of each antenna.

\section{Error Analysis}

The errors that have the largest effect are those which vary with distance, because extrapolation can amplify the effect of small errors in the data to significant errors primarily in $A_{00}^{\prime \prime}$. The magnitude of this effect is dependent on the character of the measured data and the error function. The results presented here will be for typical antennas and error functions which are usually a worst-case upper bound type. By typical antennas we mean horn and reflector type microwave antennas. 


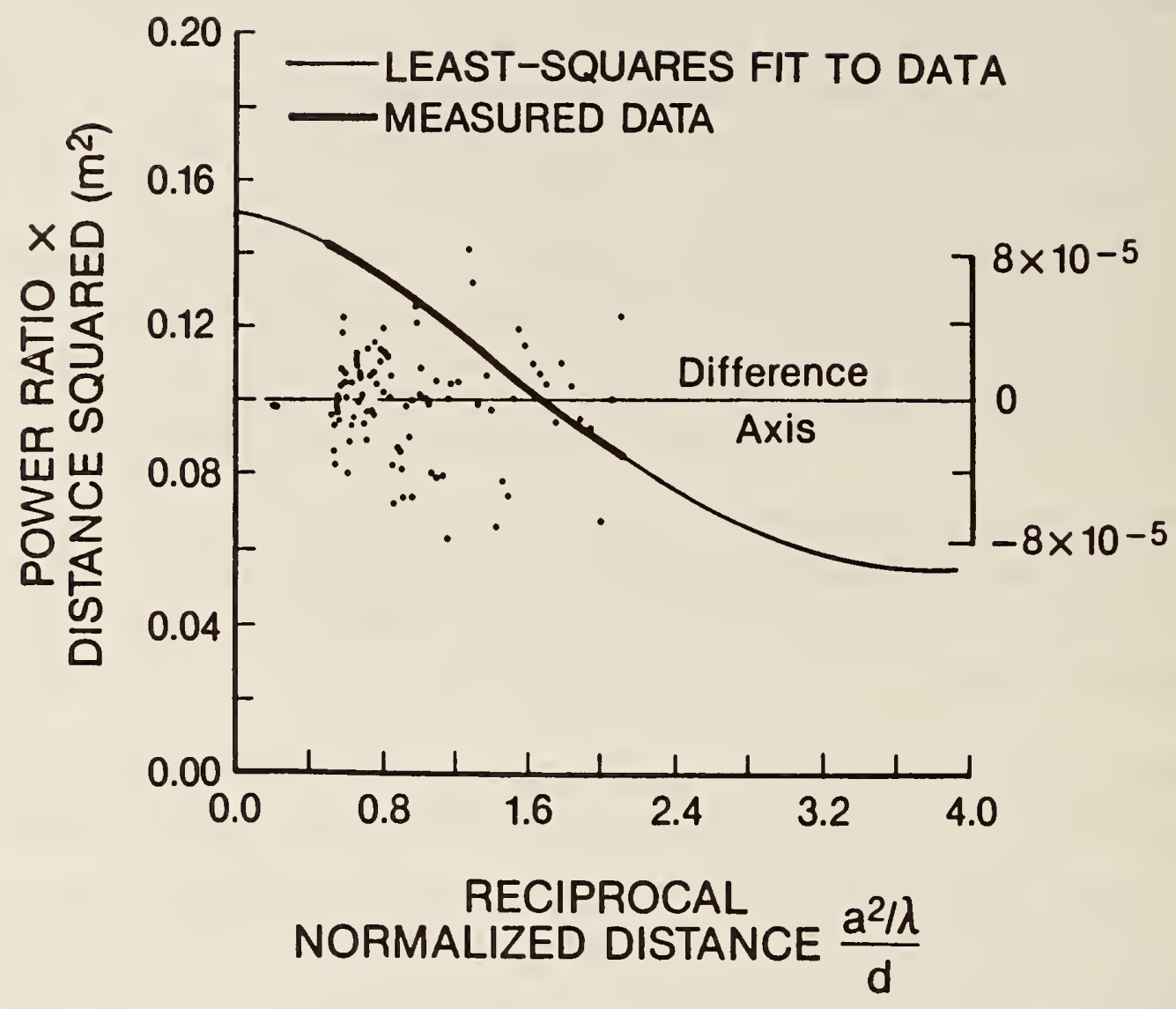

Figure 14. Measured-data, five-term polynomial fit, and residuals for $X$-band conical horn (gain $\approx 22 \mathrm{~dB}$ ).

Let us assume that the measured data consist of $\mathrm{N}$ data points equally spaced over an interval where the maximum to minimum distance ratio is denoted by $w$. The least-square fitting requires $n$ coefficients to accurately fit the data with the residual equal to $r_{n}$. The variance of $A_{0}^{\prime}$ is equal to the variance of the individual measurements (approximately $r_{n} / N-n$ ) times the first diagonal element of the inverse of the matrix of the normal equation obtained in the least squares method [22]. From empirical data this element, denoted by $C$, has been found to depend on the measurement parameters according to the equation,

$$
\log C \simeq n\left(0.3+\frac{2.4}{\omega}\right)-1-\log N .
$$


This relationship requires that $N>100, \omega>4$, and preferably that $n \leq 6$ if the effects of random error are to be minimized. Under these conditions, the standard deviation in $\mathrm{D}_{\mathrm{nm}}$ due to random error is about $0.05 \mathrm{~dB}$.

The character of errors $[23,24]$ due to receiver nonlinearity, range misalignment, instrument drift, and atmospheric attenuation uncertainty are al1 quite similar. That is, error in the amplitude data will be essentially zero for the minimum distance and can be bounded by a monotonically increasing error with a maximum value of $\varepsilon_{m}$ at the maximum distance. The continuously increasing error is a worst-case situation, and the actual error may in fact oscillate about zero which results in a smaller error in $A_{00}^{\prime}$.

For the worst-case situation, the resulting error in $D_{n m}$ due to one source with a maximum error of $\varepsilon_{m}$ is approximately $\varepsilon_{D}=2 \varepsilon_{m}$. The values of $\varepsilon_{\mathrm{m}}$ for each source (receiver, alignment, etc.) must be estimated for each measurement situation, and are typically no greater than 0.02 to $0.05 \mathrm{~dB}$.

Phase errors are caused by receiver error, antenna alignment error, and multipath signals. The largest phase errors will result when two highly linearly polarized antennas are being used. In this case, small boresight errors or inaccuracy in the 90 deg rotation can result in phase errors of tens of degrees. Fortunately, the phase is not as important in calculating gain and polarization characteristics for this case. When one antenna is linearly polarized and the other is circularly polarized, alignment is not nearly as critical, and the phase of $D_{n m}^{\prime \prime}$ can be determined with errors of 1 deg or less.

\subsection{Propagation of Errors to Gain and Polarization Parameters}

For the purposes of this discussion, we will consider $D_{n m}^{\prime}$ and $D_{n m}^{\prime \prime}$ to be quantities whose values and uncertainties are known (since they are very simply related to $A_{00}^{\prime}, A_{00}^{\prime \prime}$, and $\Delta \phi$ ) and determine the effect of these uncertainties on the computed gain and polarization parameters. This will be done for two-antenna combination sets that represent the situation for almost al1 measurements. 


\subsection{Three Nominally Linearly Polarized Antennas}

It is assumed that the axial ratio for all three antennas is at least $20 \mathrm{~dB}$ and that each antenna is oriented such that its principal component is approximately along the $y$-axis. Equation (25) for the linear components upon substituting eq (22) is then approximately

$$
\gamma_{1} \cong-i v \frac{\overline{D_{12}^{\top} D_{13}^{1}}}{D_{23}^{\prime}}, \quad x_{1} \cong \frac{-Y_{1}}{2}\left(\frac{D_{12}^{\prime \prime}}{D_{12}^{\top}}+\frac{D_{13}^{\prime \prime}}{D_{13}^{1}}-\frac{D_{23}^{\prime \prime}}{D_{23}^{T}}\right)=\frac{-Y_{1} \Delta_{1}}{2},
$$

where $\Delta_{1}$ is the quantity within the brackets of eq (42). The gain, polarization ratio, axial ratio, and tilt angle are then

$$
G_{1}=\frac{4 \pi k^{2}}{1-\left|S_{00}\right|^{2}} \quad\left\{\left|\frac{D_{23}^{\prime} D_{13}^{\prime}}{D_{23}^{\prime}}\right|\left(1+\frac{\left|\Delta_{1}\right|^{2}}{4}\right)\right\}
$$

and

$$
\rho_{\ell}=-\frac{\Delta_{1}}{2} \quad A_{1} \cong-2 / \operatorname{Im}\left(\Delta_{1}\right) \quad \tau_{1} \cong \frac{\operatorname{Re}\left(\Delta_{1}\right)}{2} .
$$

For nearly linearly polarized antennas, $\Delta_{1}$ will have little effect on the gain, and the error in $G$ will be primarily due to the errors in the $\left|D_{n m}^{\prime}\right| s$. If we let $\varepsilon_{\mathrm{mn}}$ be the error in decibels for each of the measured quantities, then the resulting error in the gain will be

$$
\varepsilon_{g}=\varepsilon_{12}+\varepsilon_{13}-\varepsilon_{23} \text {. }
$$

For the case of three antennas with approximately equal gains, the systematic error in each $\varepsilon_{\mathrm{mn}}$ will be very nearly the same. If we denote the systematic component by $\varepsilon_{s}$ and the random component by $\varepsilon_{r}$, then for this case

$$
\varepsilon_{g}=\varepsilon_{s}+\sqrt{3} \varepsilon_{r}
$$

For the axial ratio and tilt angle, errors in $\Delta_{1}$ have the major effect. If we denote the amplitude and phase of the ratios in $\Delta_{1}$ as

$$
\frac{D_{n m}^{\prime \prime}}{D_{n m}^{\prime}}=a_{n m} e^{i \theta_{n m}},
$$


then the errors in $A$ and $\tau$ will be given by

$$
\begin{aligned}
d A_{1} \cong & -\frac{A_{1}^{2}}{2}\left(a_{12} \cos \theta_{12} d \theta_{12}+a_{13} \cos \theta_{13} d \theta_{13}-a_{23} \cos \theta_{23} d \theta_{23}\right. \\
& \left.+\sin \theta_{12} d a_{12}+\sin \theta_{13} d a_{13}-\sin \theta_{23} d a_{23}\right)
\end{aligned}
$$

and

$$
\begin{aligned}
d \tau & \cong a_{12} \sin \theta_{12} d \theta_{12}+a_{13} \sin \theta_{13} d \theta_{13}-a_{23} \sin \theta_{23} d \theta_{23} \\
& -\cos \theta_{12} d a_{12}-\cos \theta_{13} d a_{13}+\cos \theta_{23} d a_{23} .
\end{aligned}
$$

5.3 Two Nominally Linearly and One Nominally Circularly Polarized

Antenna Combination

The linearly polarized antennas are again oriented with their principal components approximately along the $y$-axis, and we assume that antenna number 3 is nearly left circularly polarized. When either antenna 1 or 2 is used with number 3 , the 90 deg rotation about the z-axis will result in a very small amplitude change, and approximately $90 \mathrm{deg}$ phase change. Therefore,

$$
\frac{D_{12}^{\prime \prime}}{D_{12}^{\prime}}=\delta_{12}, \frac{D_{13}^{\prime \prime}}{D_{13}^{\prime}}=-i+\delta_{13}, \frac{D_{23}^{\prime \prime}}{D_{23}^{\prime}}=-i+\delta_{23},
$$

where the $\delta$ 's represent the small deviation from exactly linear and circular polarization. An analysis similar to that in the previous section shows that the left and right components of $S_{10}(0)$ are given approximately by

$$
L_{3} \cong \pm \frac{4 D_{13}^{1} D_{23}^{\prime}}{D_{12}^{1}}, \quad R_{3} \cong \pm \frac{L_{3}}{2} \sqrt{-\delta_{13} \delta_{23}},
$$

and that the gain, circular polarization ratio, axial ratio, and tilt angle are approximately

$$
G_{3} \simeq \frac{4 \pi k^{2}}{1-\left|S_{00}\right|^{2}} \frac{\left(\left|D_{13}^{\prime}\right|+\left|D_{13}^{\prime \prime}\right|\right)\left(\left|D_{23}^{\prime}\right|+\left|D_{23}^{\prime \prime}\right|\right)}{2\left|D_{12}^{\prime}\right|}
$$

and 


$$
\rho_{C} \cong \frac{\sqrt{-\delta_{13} \delta_{23}}}{2} \quad A \cong-\left(1+\left|\sqrt{\delta_{13} \delta_{23}}\right|\right) \quad \tau=\frac{\pi+\phi_{13}+\phi_{23}}{4},
$$

where $\phi_{13}$ and $\phi_{23}$ are the phase angles of $\delta_{13}$ and $\delta_{23}$.

The gain calculation here is similar to the previous case, where only the large quantities were significant. There is once again some cancellation of the systematic error components for this case, but since five rather than three quantities are involved, the effect of random error is larger. The gain error is given by $\varepsilon_{g}=\varepsilon_{s}+\sqrt{5} \varepsilon_{r}$ where $\varepsilon_{s}$ and $\varepsilon_{r}$ are the systematic and random errors in each of the $D_{n m}{ }^{\prime} s$.

If two similar linear antennas are used, then $\delta_{13} \cong \delta_{23}$ and for this case the error in the axial ratio is

$$
d A \cong\left(2 a_{13}+2 \sin \theta_{13}\right) d a_{13}+2 a_{13} \cos \theta_{13} d \theta_{13} .
$$

Since $\theta_{13} \cong-90^{0}$ and $a_{13} \cong 1$, errors $\left(d_{13} \approx 0\right)$ in the measured quantities do not strongly affect the calculated axial ratio.

The accuracy of tilt angle is very dependent on experimental errors, especially for highly circular polarization. The angles $\theta_{13}$ and $\theta_{23}$ are related to the measured ratios by

$$
\phi_{13}=\tan ^{-1}\left(\frac{1+a_{13} \sin \theta_{13}}{a_{13} \cos \theta_{13}}\right) \quad \phi_{23}=\tan ^{-1}\left(\frac{1+a_{23} \sin \theta_{23}}{a_{23} \cos \theta_{23}}\right),
$$

and the error in $\phi_{13}$ is

$$
d \phi_{13}=\frac{-\cos \theta_{13} d a_{13}+\left(a_{13}^{2}+a_{13} \sin \theta_{13}\right) d \theta_{13}}{1+a_{13}^{2}+2 a_{13} \sin \theta_{13}} \text {. }
$$

The tilt angle error is given by

$$
d \tau \cong \frac{d \phi_{13}+d \phi_{23}}{4}
$$

and can be quite large for nearly circular polarization when $\theta_{13}$ and $\theta_{23}$ are close to -90 deg and $a_{13}$ and $a_{23}$ are nearly 1 . 


\section{Measurement Examples}

The extrapolation technique has been used on a number of antennas at frequencies from 1 to $65 \mathrm{GHz}$. For some of these, gain measurements have been made using different techniques and the results have always agreed to within $\pm 0.15 \mathrm{~dB}$. In one comparison, the gain of a multimode $X$-band horn was measured by NBS using both the extrapolation technique and a near-field scanning technique. The Jet Propulsion Labs also determined the gain of the horn [25] from far-field pattern measurements. The results of all three measurements were within $\pm 0.10 \mathrm{~dB}$ of each other.

For some measurements on pyramidal horns, the proximity corrections obtained from the extrapolation measurements had been compared with theoretical expressions based on the geometry of the horn and assumed field distributions. There has been fairly good agreement with the results of chu and Semplak [26] for a limited number of comparison.

The results of measurements on two different antenna sets will now be described. These will illustrate the present accuracy capabilities for antenna gain and polarization measurements.

\subsection{X-Band Standard Gain Horns}

This antenna set consisted of two commercial standard gain horns with nearly linear polarization and one horn with nominally circular polarization. The measurement frequency was $7.250 \mathrm{GHz}$, and $a^{2} / \lambda$ for the circularly polarized horn was about $1.33 \mathrm{~m}$. Measurements were made over a distance range from 1.2 to $2.8 \mathrm{~m}$ for the three antenna combinations and the extrapolation procedure carried out to give the results shown in table 1 which also includes the $3 \sigma$ error estimates. The error estimates for atmospheric loss uncertainty are obtained from reference [27]. The measured data and error estimates then result in the following calculated quantities for the circularly polarized horn:

$$
\begin{aligned}
& \text { Gain }=21.32 \mathrm{~dB} \pm 0.11 \mathrm{~dB} \\
& \text { Axial ratio }=1.34 \mathrm{~dB} \pm 0.05 \mathrm{~dB} \\
& \text { Tilt angle }=-59.3^{\circ} \pm 2.5^{\circ}
\end{aligned}
$$


Table 1. Data and errors for X-band antennas.

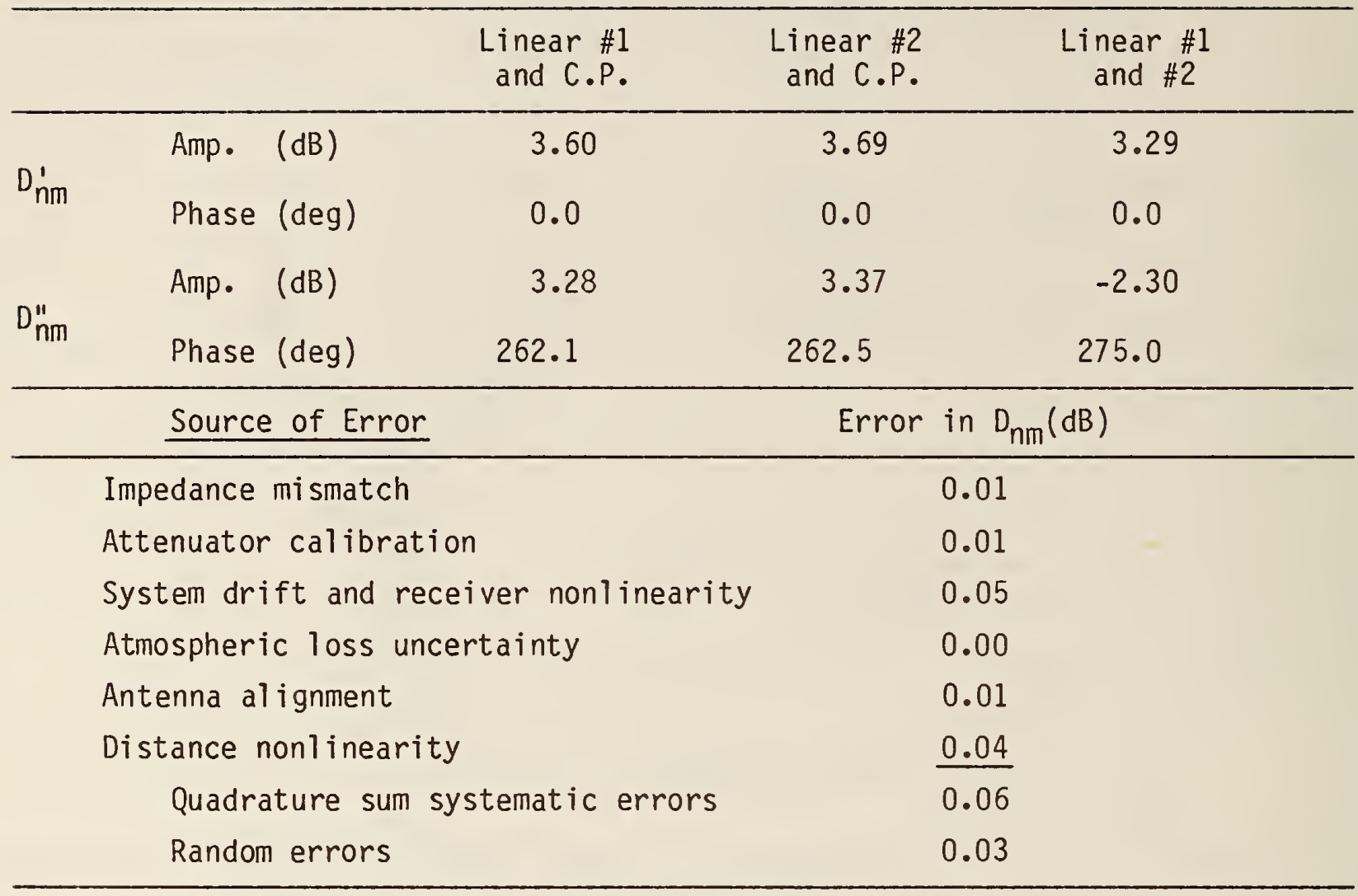

\subsection{V-Band Reflector Antennas}

These were linearly polarized, $45 \mathrm{~cm}$ diameter reflector antennas. Antennas 1 and 2 were equipped with focal point feeds and \#3 has a Cassegrain feed. At the test frequency of $57.5 \mathrm{GHz}, a^{2} / \lambda$ was $40 \mathrm{~m}$. Extrapolation data was taken over a range from 10 to $60 \mathrm{~m}$ on the NBS outdoor range with the results shown in table 2 .

From these data the antenna parameters and error estimates were determined to be
Antenna
$\# 1$
$\# 2$
$\# 3$
Gain (dB)
$46.61 \pm 0.23 \cdot 46.59 \pm 0.23$
$46.26 \pm 0.23$
Axial ratio $(d B)$
$35.1 \pm 2.4$
$43.7 \pm 3.3$
$26.7 \pm 1.2$
Tilt angle (deg)
$87.6 \pm 0.2$
$88.6 \pm 0.1$
$-89.0 \pm 0.5$ 
Table 2. Data and errors for mm-wave antennas.

\begin{tabular}{|c|c|c|c|c|}
\hline & & $\# 1$ and \#2 & $\# 2$ and \#3 & $\# 1$ and \#3 \\
\hline \multirow{2}{*}{$D_{n m}^{\prime}$} & Amp. $(d B)$ & -26.06 & -26.23 & -26.21 \\
\hline & Phase (deg) & 0.0 & 0.0 & 0.0 \\
\hline \multirow{2}{*}{$D_{n m}^{\prime \prime}$} & Amp. $(d B)$ & -37.56 & -40.19 & -40.49 \\
\hline & Phase (deg) & 20.0 & 280.0 & 310.0 \\
\hline \multicolumn{3}{|c|}{ Source of Error } & \multicolumn{2}{|c|}{ Error in $D_{n m}(d B)$} \\
\hline \multicolumn{3}{|c|}{ Impedance mismatch } & \multicolumn{2}{|c|}{0.01} \\
\hline \multicolumn{3}{|c|}{ Attenuator calibration } & \multicolumn{2}{|c|}{0.006} \\
\hline \multicolumn{3}{|c|}{ System drift and receiver nonlinearity } & \multicolumn{2}{|c|}{0.05} \\
\hline \multicolumn{3}{|c|}{ Atmospheric loss uncertainty } & \multicolumn{2}{|c|}{0.10} \\
\hline \multicolumn{3}{|c|}{ Antenna alignment } & \multicolumn{2}{|c|}{0.03} \\
\hline \multicolumn{3}{|c|}{ Distance nonlinearity } & \multicolumn{2}{|c|}{$\underline{0.04}$} \\
\hline \multirow{2}{*}{\multicolumn{3}{|c|}{$\begin{array}{l}\text { Quadrature sum systematic errors } \\
\text { Random errors }\end{array}$}} & \multicolumn{2}{|c|}{0.12} \\
\hline & & & \multicolumn{2}{|c|}{0.07} \\
\hline
\end{tabular}

7. Improved Polarization Measurements

Absolute polarization parameters have been obtained in the previous sections using a three-antenna measurement technique $[12,13,28]$. By a modification of this approach [29], the accuracy of the results are significantly increased, and the measurements are easier to perform. Using this technique, it is possible to accurately calibrate polarization standards which have axial ratios greater than $40 \mathrm{~dB}$. These standards are an important factor in the measurement of antenna systems which require high polarization purity. This work demonstrates the power and utility of using complex rather than real polarization parameters in formulating polarization problems.

As we did before and repeat here for convenience, let us define three coordinate systems, one fixed to each of two antennas and one fixed in space and referenced to the test range. Each antenna system is defined with the boresight direction along the $z$-axis and the $y$-axis nominally along the major 
axis of the polarization ellipse. The exact location of axes is not critical but it is important that they be clearly defined. The reference, or space, coordinate system has its $x$-axis horizontal, its $y$-axis vertical, and its $z-$ axis along the center of the test range. Now place the source antenna on the test range so that its axes are coincident with the reference system. The receiving antenna has its boresight (+z-axis) along the reference-z-direction, and is rotated about the reference $z$-axis into two orientations where measurements are performed (fig. 15). In the first orientation the $+y$-axes of the antennas are in the same direction, and in the second the $-x$ of the receiving antenna is in the same direction as the ty of the source antenna. The coupling equations for these two orientations [eq (21)] are respectively

$$
\begin{aligned}
& X_{1} X_{2}-Y_{1} Y_{2}=D_{12}^{\prime}, \\
& x_{1} Y_{2}+X_{2} Y_{1}=D_{12}^{\prime \prime}
\end{aligned}
$$

where the D's are proportional to the complex received signals, and $X_{1}$ and $Y_{1}$ are the $x$ - and $y$-components of the plane-wave spectrum transmitting characteristic $\underline{S}_{10}(\theta, \phi)$ of the source antenna in the direction $\theta=\phi=0$. $X_{2}$ and $Y_{2}$ are similar quantities for the receiving antenna. $\underline{S}_{10}(\theta, \phi)$ is simply related to the far-electric field $E$, [eq (9)] and since we will only be concerned here with ratios of the components, the formulation can be thought of as being in terms of either $\underline{E}$ or $\underline{S}$.

In eqs (55) and (56) we have assumed that the receiving antenna is reciprocal and expressed all antenna parameters in terms of transmitting properties. This is not necessary to the technique, but it makes the notation more concise dild consistent with previous material [13].

The approach in the three-antenna measurement is to repeat this procedure for all three antenna pairs, measure the $D_{n m}$ 's and solve the resulting six equations for the linear components $X_{n}$ and $Y_{n}$, the right and left circular components $R_{n}$ and $L_{n}$, and obtain gain, complex linear polarization ratio $\rho_{\ell}$, complex circular polarization ratio $\rho_{C}$, axial ratio $A$, and the tilt angle $\tau$. These polarization parameters are related by the equations [eqs (13) through (18)] 


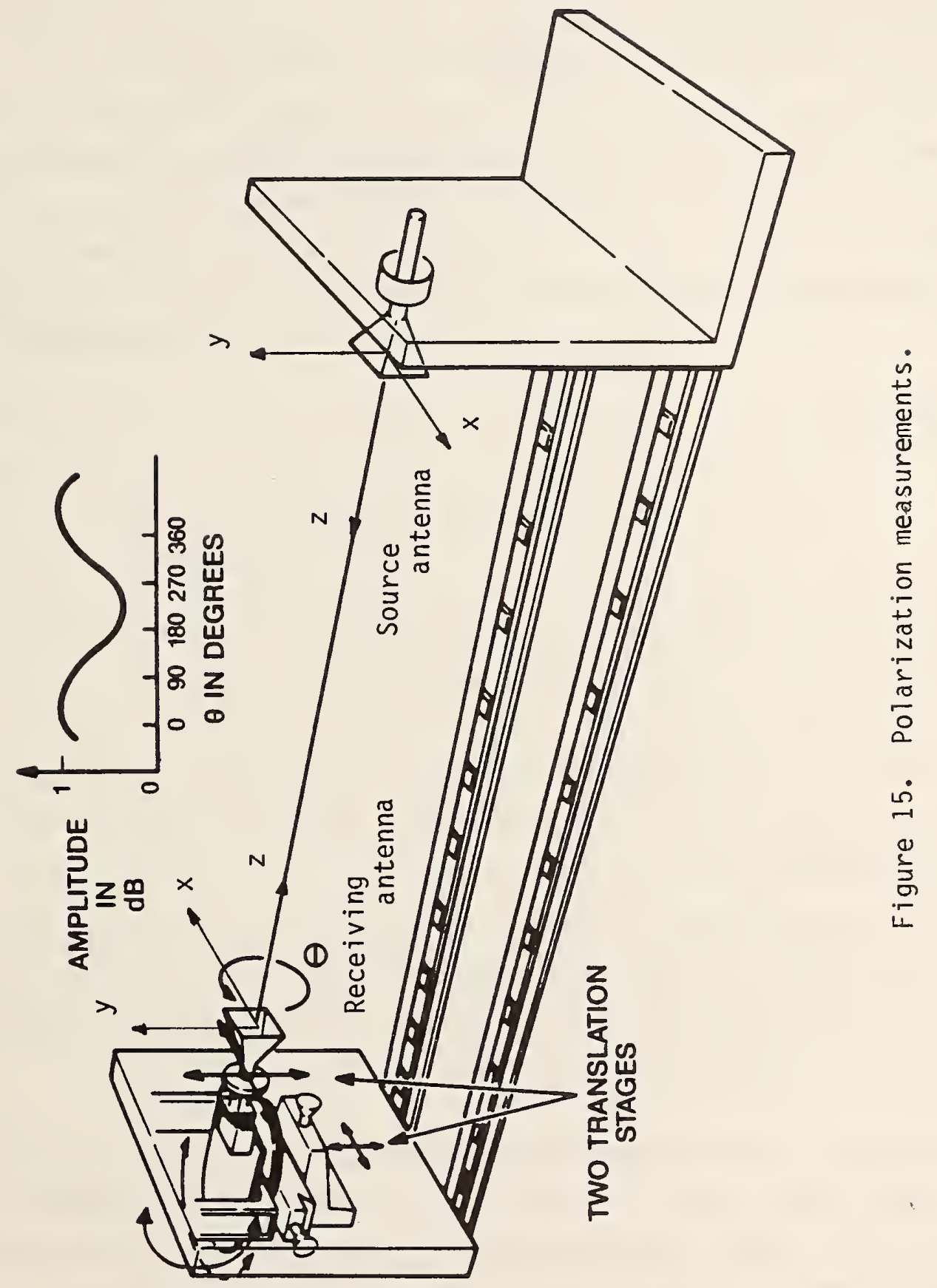




$$
\begin{aligned}
& R=\frac{X-i Y}{\sqrt{2}}, L=\frac{X+i \gamma}{\sqrt{2}}, X=\frac{R+L}{\sqrt{2}}, Y=\frac{i(R-L)}{\sqrt{2}}, \\
& \rho_{\ell}=\frac{X}{Y}, \rho_{C}=\frac{L}{R}, A=\frac{|R|+|L|}{|R|-|L|}, \tau=\frac{\arg \left(\rho_{C}\right)}{2} .
\end{aligned}
$$

All four of the vector components are, in general, complex quantities, and $X$ and $Y$ do not represent real and imaginary parts. Also the measured linear components are those for the reference coordinate system and all of the quantities in eqs (39) and (40) except A will change if the initial orientation of the antennas is changed.

For polarization measurements, only the ratios of the components and the received signals are necessary and we can combine eqs (56) and (57) and express the result for the general case of antennas $m$ and $n$ in terms of linear and circular polarization ratios.

$$
\begin{gathered}
\frac{\rho_{\ell m}+\rho_{\ell n}}{\rho_{\ell m} \rho_{\ell n}-1}=Q_{n m}=\frac{D_{n m}^{\prime \prime}}{D_{n m}^{1}}, \\
\rho_{c m} \rho_{c n}=\frac{1+i Q_{n m}}{1-i Q_{n m}}=\frac{1-i P_{m n}}{1+i P_{m n}} .
\end{gathered}
$$

In the above equation, $P_{n m}$ is the ratio of $D_{n m}^{\prime \prime} / D_{n m}^{\prime}$ if the receiving antenna is rotated in the opposite direction for the second orientation, placing its $+x-$ axis coincident with the source antenna's +y-axis. The major source of error in the polarization parameters obtained from these measurements is the uncertainty in the phase of $Q_{n m}$. For two nearly linearly polarized antennas, the magnitude of $Q_{n m}$ may be -30 to $-60 \mathrm{~dB}$ and at these levels, multipath signals, depolarization of scattered signals, and leakage make accurate phase measurements difficult. The phase will also change very rapidly as the 90 deg rotation is approached; this makes it necessary to measure the rotation angle quite accurately. The uncertainty in the phase of the Q's can be reduced essentially to zero by the following modification of the measurement.

We first note from eq (57) and the definition of $\tau$ that $\tau= \pm \pi / 2$, if and only if $\rho_{c}$ is negative and real, arg $\left(\rho_{\ell}\right)= \pm \pi / 2$, and the major axis of the polarization ellipse is exactly along the $y$-axis of the reference coordinates. 
We can further show that $\left|Q_{m n}\right|$ will be a minimum if the major axes are initially coincident, and under this condition, the phase of $Q_{n m}$ must be precisely $\pm \pi / 2$. If we can initially align the antennas so that their major axes are parallel, the phase measurement will reduce to one of determining the sign of the phase. The initial alignment can be accomplished quite easily by rotating the source antenna about the reference $z$-axis for a minimum received signal and then rotating back $90 \mathrm{deg}$ from that orientation.

The sign of the phase change can be determined by observing the direction of phase change as the receiving antenna is rotated towards $90 \mathrm{deg}$. It can be shown using eq (55) expressed in terms of circular components that for the previously assumed conditions, the phase change of $D_{n m}^{\prime}$ denoted by $\psi$, for any rotation angle $\phi$, is

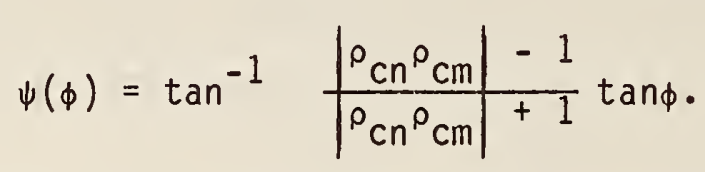

Therefore, if the argument of $Q_{n m}$ is $+\pi / 2$, the phase change will be positive for all angles between 0 and $90 \mathrm{deg}$ and the converse will be true for a $-\pi / 2$ argument.

In summary, the measurement procedure is to initially align the antennas with their chosen $y$-axes parallel, set the angle encoders to 0 deg for this orientation, and rotate the receiving antenna in the proper direction about the $z$-axis until a minimum is found, and then rotate $90 \mathrm{deg}$ in the opposite direction. In this orientation the amplitude is set to 1.0 , the phase to $0 \mathrm{deg}$, and the rotation angle $\Delta \phi_{\mathrm{nm}}$ is recorded. The receiving antenna is again rotated to the minimum, the direction of phase change during rotation is noted, and the amplitude at the minimum, $\alpha_{n m}$ is recorded. $Q_{n m}$ is then equal to $\pm i a_{n m}$ where the correct sign is equal to the direction of phase change. This procedure is repeated for the other two antenna pairs, and eq (60) solved for the polarization ratios.

By aligning the antennas so that their electrical axes are parallel we have essentially redefined the reference coordinate system to be coincident with the electrical axes of the source antenna. With this redefinition, $A=1 /\left|\rho_{\ell}\right|$ for both antennas. 
The tilt angle with respect to the antenna axes is determined from the measured rotation angles $\Delta \phi_{\mathrm{nm}}$ by the equation,

$$
\tau_{k}=90^{\circ}+\frac{\Delta \phi_{k m}+\Delta \phi_{k n}-\Delta \phi_{m n}}{2} .
$$

This technique is especially useful in the calibration of linear polarization standards with very small cross polarization. In this case, the magnitude of the denominator of eq (59) is very nearly 1 and a quick calculation yields the linear polarization ratios. For instance,

$$
\rho_{\ell 1} \simeq-\left(Q_{12}+Q_{13}-Q_{23}\right) / 2
$$

This technique was used on a set of four Ku-band standard gain horns where all combinations were measured to give three sets of data from which each antenna's polarization could be computed. The consistency of the results shown in table 3 indicates the accuracy of the technique.

We have discussed the case where the polarization is measured in the boresight directions (along the antenna z-axis), but the same procedure can be used for any direction. By rotating one or both of the antennas so that a direction other than boresight is along the space z-axis, the spherical components in that direction will be determined. Rotation of the receiving antenna in the measurement of $Q_{n m}$ must be about the space z-axis which may not be the antenna $z$-axis in this case.

Table 3. Results of polarization measurements.

\begin{tabular}{lcccccccc}
\hline $\begin{array}{c}\text { Antenna } \\
\text { combinations }\end{array}$ & $\begin{array}{c}\mathrm{A}_{1} \\
(\mathrm{~dB})\end{array}$ & $\begin{array}{c}\tau_{1} \\
(\mathrm{deg})\end{array}$ & $\begin{array}{c}\mathrm{A}_{2} \\
(\mathrm{~dB})\end{array}$ & $\begin{array}{c}\tau_{2} \\
(\mathrm{deg})\end{array}$ & $\begin{array}{c}\mathrm{A}_{3} \\
(\mathrm{~dB})\end{array}$ & $\begin{array}{c}\tau_{3} \\
(\mathrm{deg})\end{array}$ & $\begin{array}{c}\mathrm{A}_{4} \\
(\mathrm{~dB})\end{array}$ & $\begin{array}{c}\tau_{4} \\
(\mathrm{deg})\end{array}$ \\
\hline $1,2,3$ & 67.7 & 89.62 & 54.8 & 89.84 & 47.1 & 89.83 & & \\
$1,2,4$ & 73.9 & 89.60 & 53.9 & 89.86 & & & 60.0 & 89.86 \\
$1,3,4$ & 70.3 & 89.68 & & & 46.8 & 89.79 & 61.0 & 89.80 \\
$2,3,4$ & & & 54.4 & 89.80 & 47.3 & 89.77 & 59.1 & 89.82 \\
\hline
\end{tabular}


The accuracy of the results are primarily dependent on the measurement of the magnitude of the Q's. If care is taken to average the effect of multipath by moving one antenna a small distance in the z-direction, the errors in $A$ are on the order of $\pm 0.05 \mathrm{~dB} / \mathrm{dB}$ of axial ratio, and about \pm 0.2 deg in $\tau$.

\section{Swept Frequency Measurement Techniques}

Careful measurements of gain versus frequency of standard gain horns exhibit small periodic amplitude oscillations of the order of 0.1 to $0.2 \mathrm{~dB}$ superimposed on a smooth monotonic gain function. These oscillations are attributed to multiple reflections between the mouth and throat of the horn [7]. For highest accuracy, the gain must be determined at closely spaced frequencies and swept frequency techniques may be used. The parameters are measured as a function of frequency and the values are adjusted at specific frequencies to agree with the more accurate but time consuming extrapolation measurements. The resulting gains as a function of frequency are fully corrected for mismatch, near-zone effects and multiple reflections between antennas. The accuracy obtainable over the band will typically be of the order of $\pm 0.15 \mathrm{~dB}$.

As implied earlier, the extrapolation method is used as the basis for accurate swept (stepped) frequency gain calibrations (fig. 16). To accomplish this, fixed frequency extrapolation measurements are usually performed at the end and mid-points of a waveguide band. Next, swept measurements of the pertinent reflection coefficients (both amplitude and phase) are taken looking into (1) generator port, (2) transmitting antenna, (3) receiving antenna, and (4) load port. Examples are given in figures 17 through 20. From these values, the mismatch correction is calculated as a function of frequency (fig. 21).

The next step in taking swept frequency measurements is to determine the z-distance at which the measurements will be taken. This is done by examining the extrapolation data and determining the distance where the insertion loss is approximately -10 to $-12 \mathrm{~dB}$. This usually assures that the distance is about 1 to $2 a^{2} / \lambda$, which is sufficient for currently specified error uncertainties. This distance also ensures that there is a measurable signal across the band. It is at this distance that the near-zone correction is obtained 


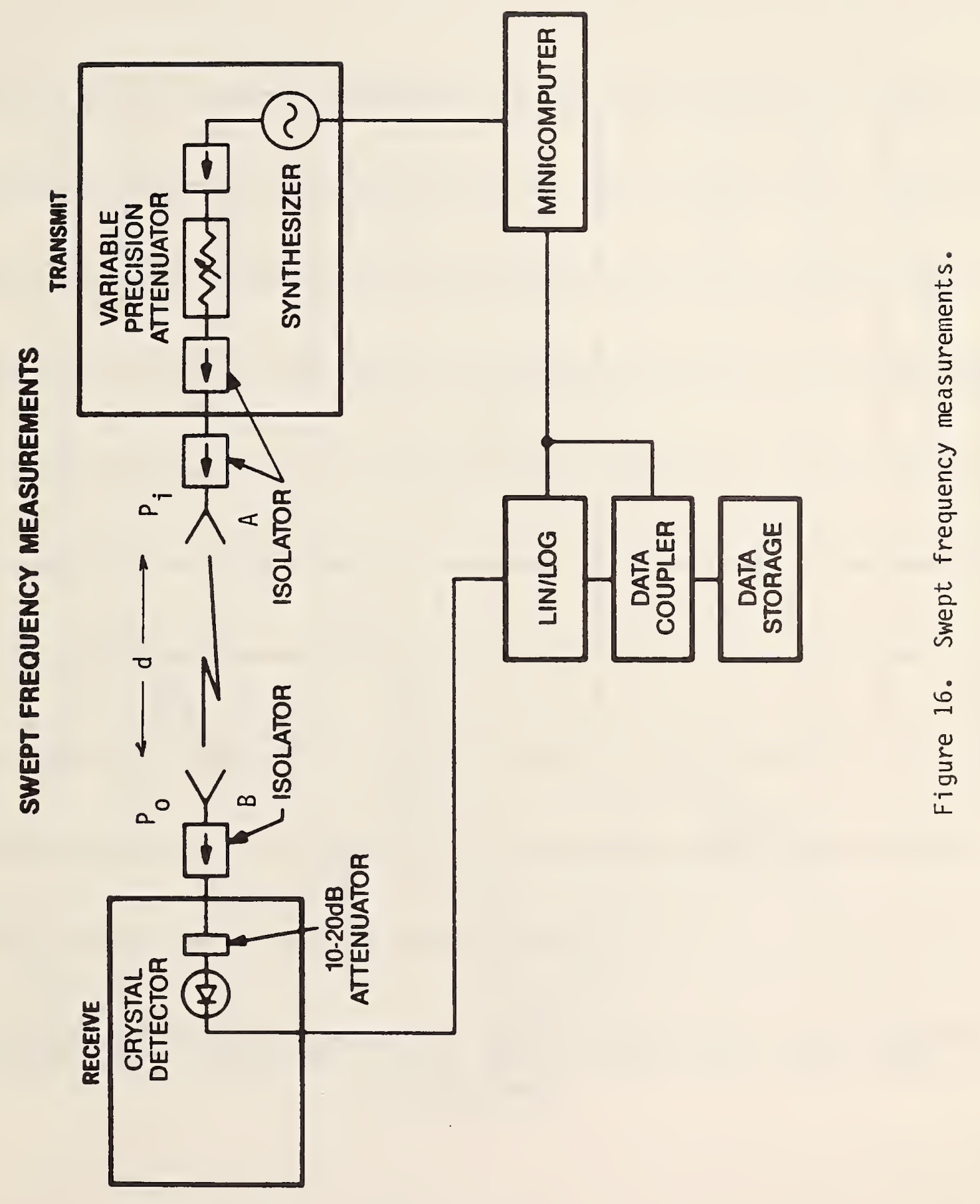




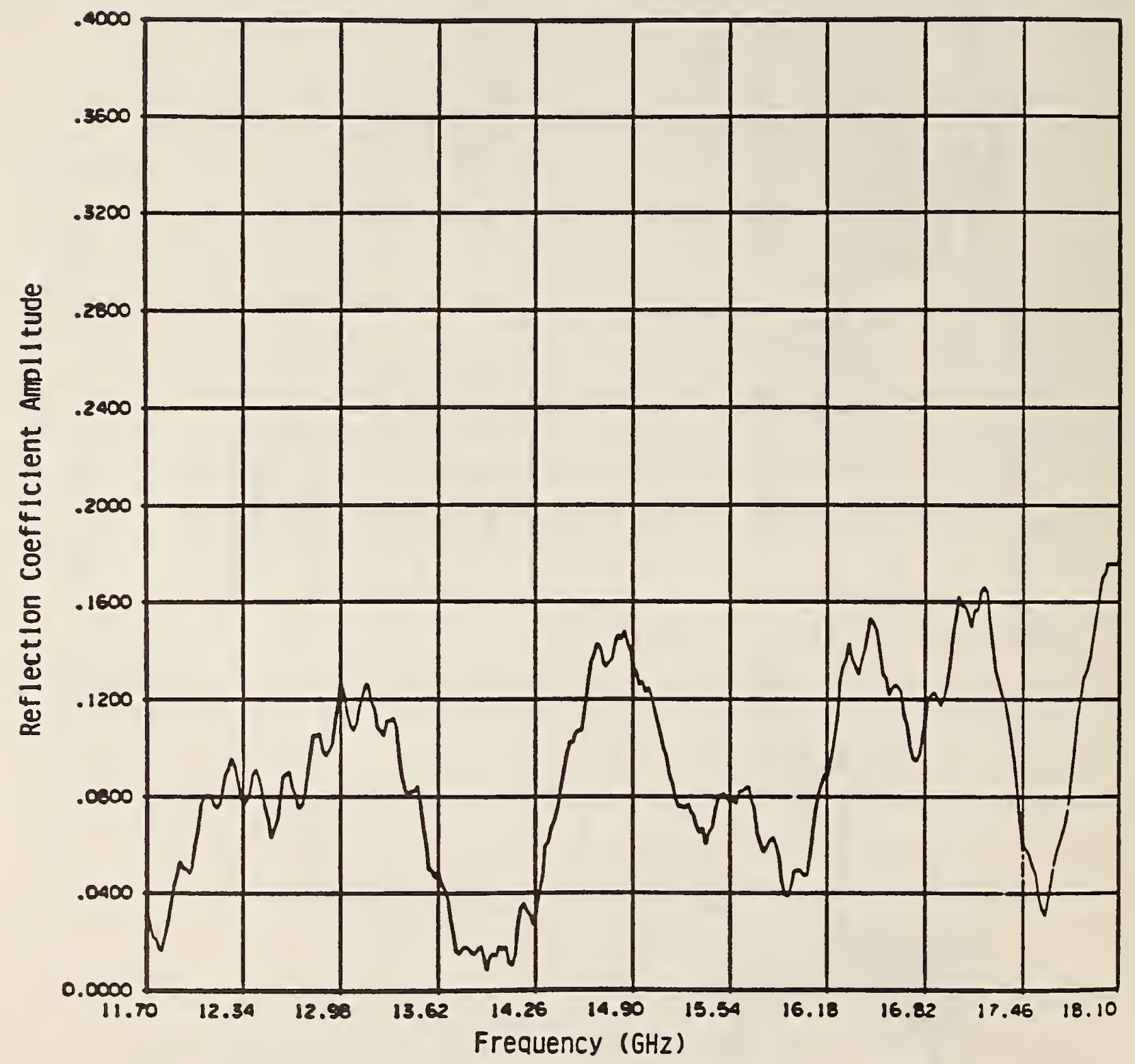

Figure 17. Swept frequency, reflection coefficient measurement (amplitude) for generator port. 


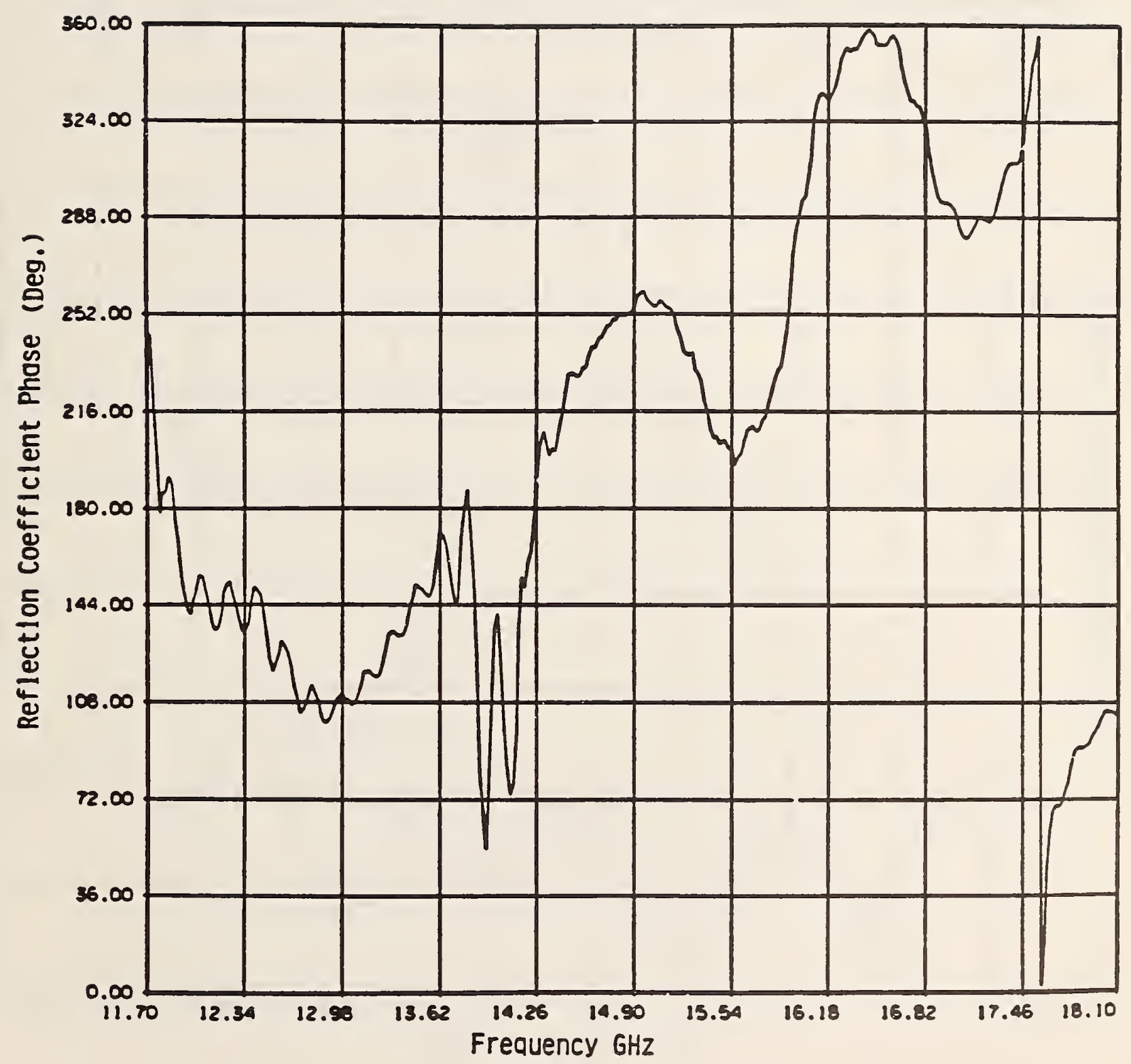

Figure 18. Swept frequency, reflection coefficient measurement (phase) for generator port. 


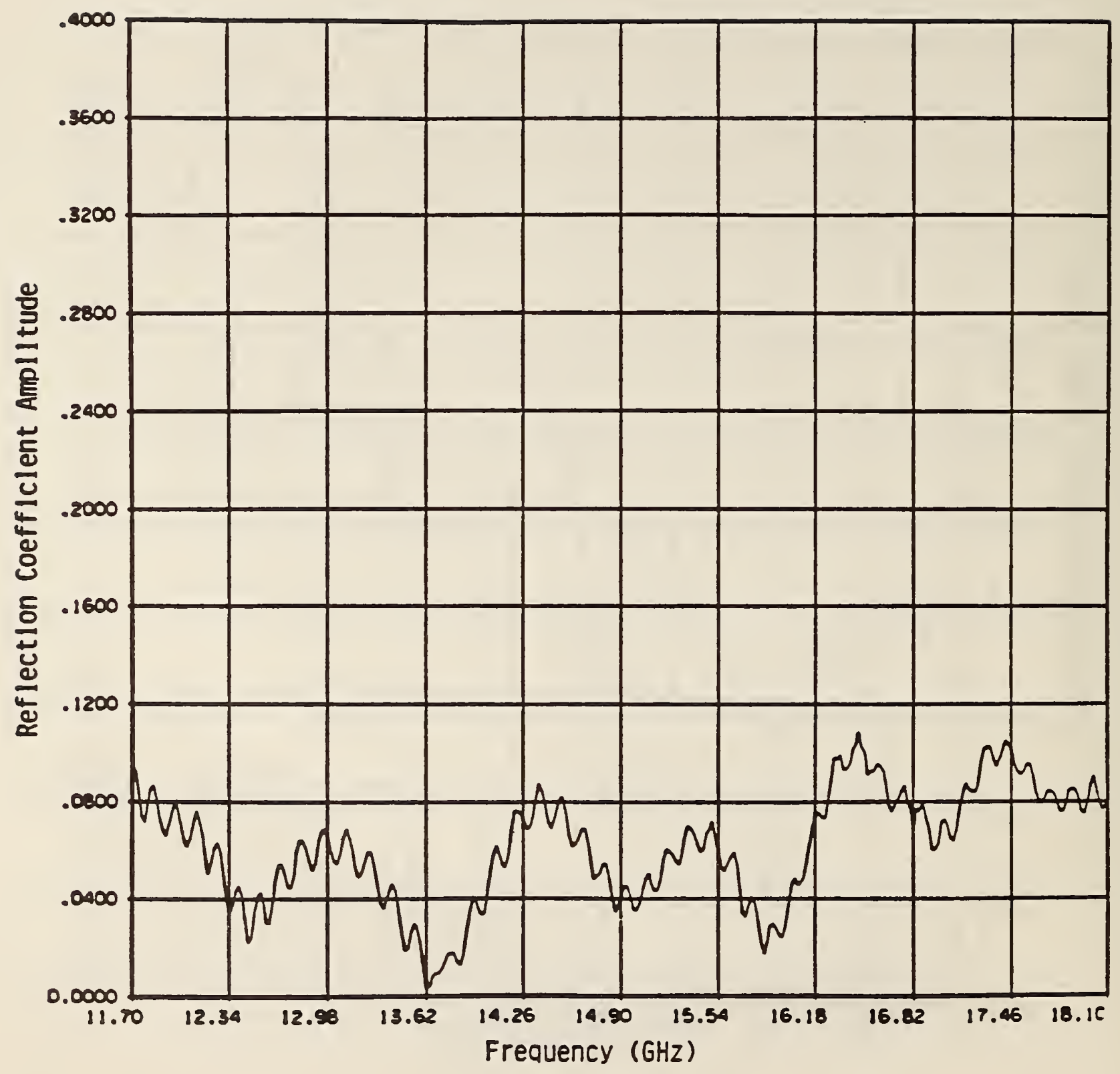

Figure 19. Swept frequency, reflection coefficient measurement (amplitude) for a long pyramidal horn. 


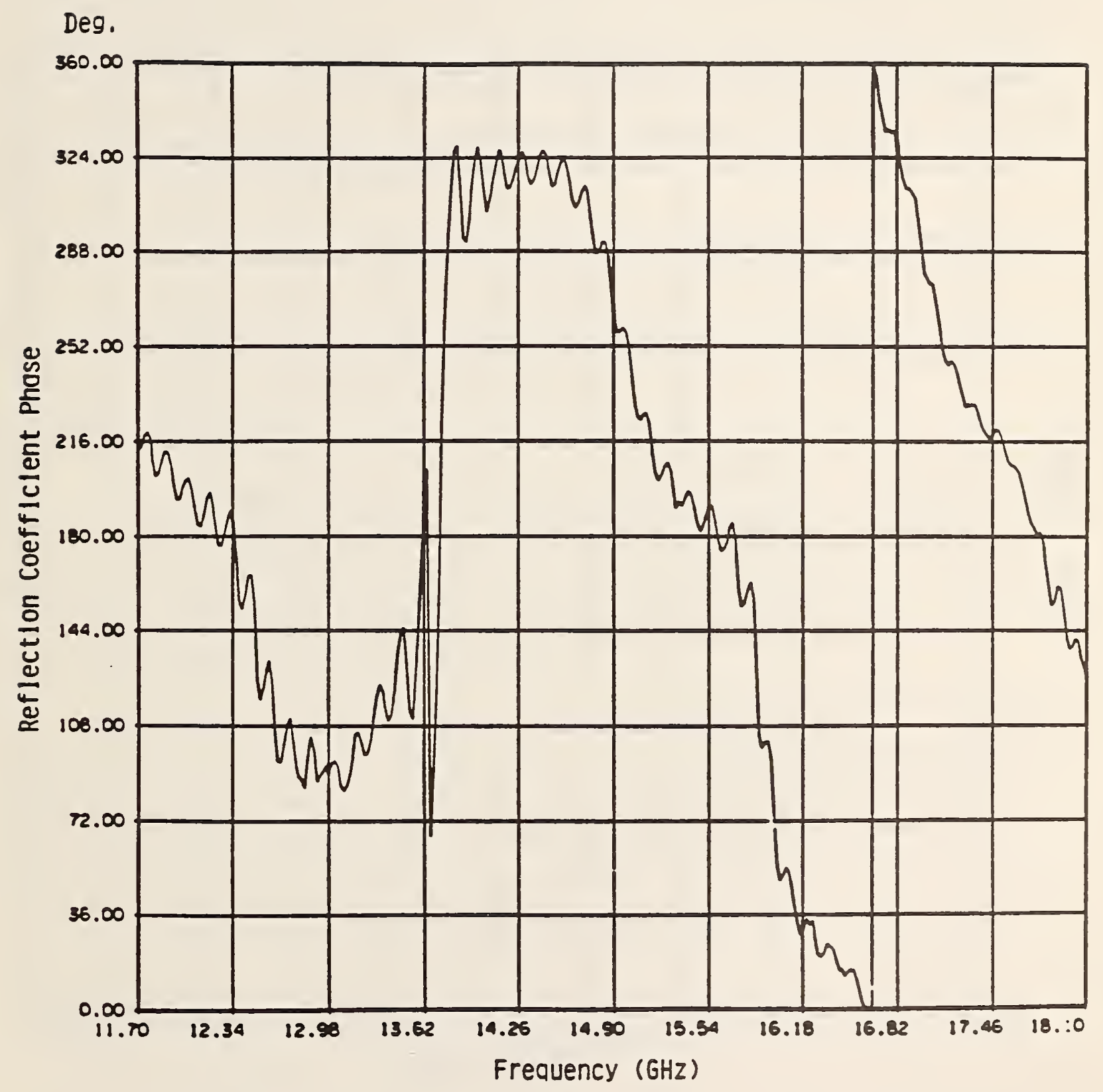

Figure 20. Swept frequency, reflection coefficient measurement (phase) for a long pyramidal horn. 


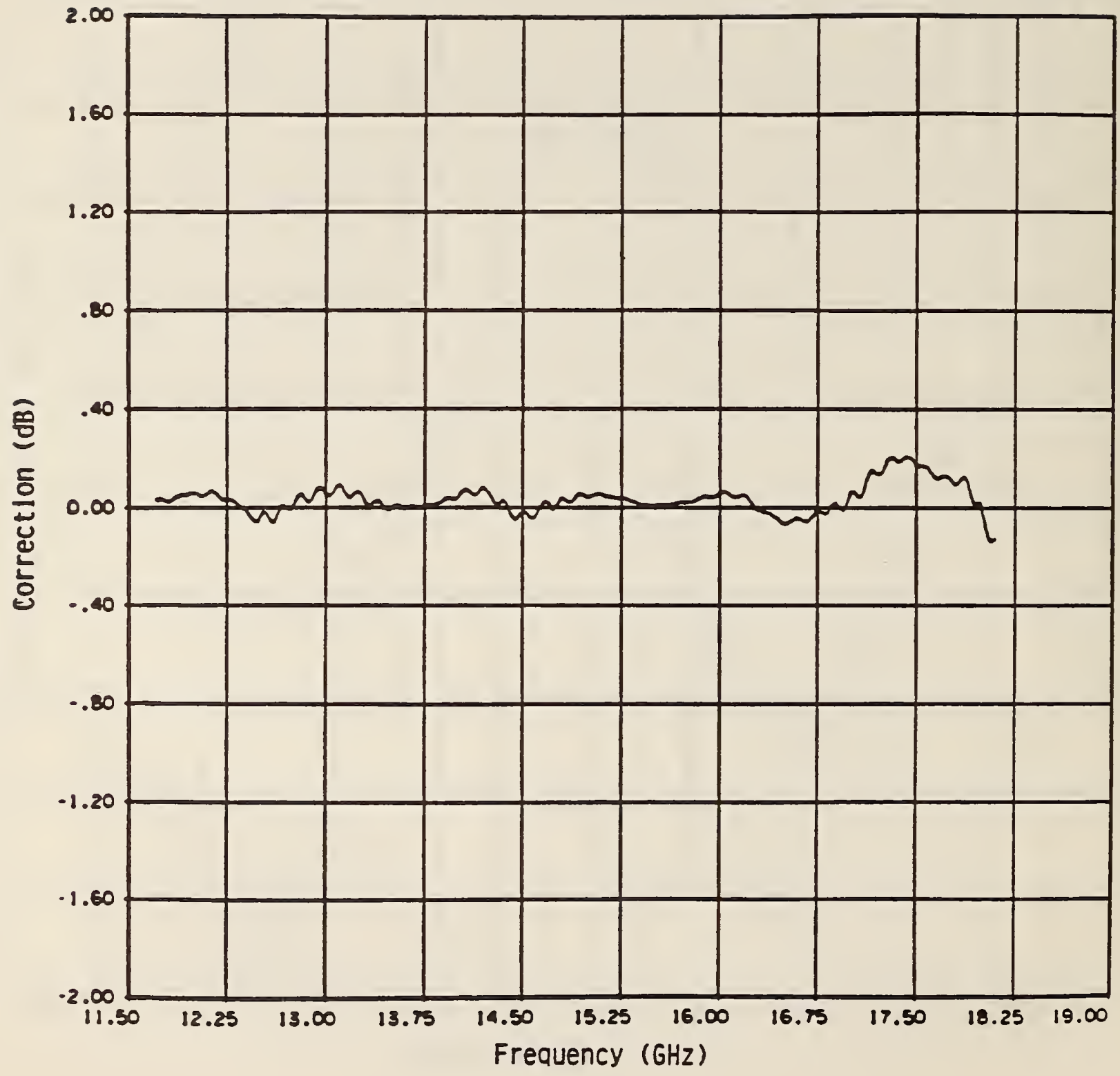

Figure 21. Mismatch correction calculated from measured reflectioncoefficient data. 
from the fixed-frequency extrapolation measurements (fig. 22). The receiving system is then initialized by requiring that the receiver is operating in its linear range and that the analog-to-digital amplitude meter is within its operating range $(0$ to $-20 \mathrm{~dB})$. The computer is then programmed to step (sweep) the signal source from $f_{\min }$ to $f_{\max }$ in steps small enough to assure that small variations in antenna gain are measured. Usually, $f_{\min }$ and $f_{\max }$ are chosen to be the lowest and highest frequencies at which extrapolation range gain measurements at fixed frequencies were taken. Frequency and amplitude data are then recorded (fig. 23).

The towers are then brought together and a generator-load connection is made. A recorded offset has been inserted on the precision rotary vane attenuator (RVA) to reduce the power level and again insure proper receiver system operation. Frequency and amplitude data for this hookup are then recorded (fig. 24).

After this procedure is checked out, the measurement is redone at least twice for each pair of antennas. The sequence becomes first to measure the generator-load connection, followed by reinstalling the horns, removing the RVA offset, moving the towers to the appropriate distance and then recording the frequency and amplitude measurements.

We now have sufficient information to determine the gain as a function of frequency, fully corrected for mismatch, near zone, and multiple reflection effects between antennas. Figure 25 shows a typical insertion loss result including mismatch and near-zone corrections for a pair of Ku band horns. This insertion loss is the difference between the generator-load reference curve and the insertion loss curve with the antennas in $\mathrm{place}$, both mentioned above. The high frequency oscillations are due to multiple reflections between the antennas. The effects can be either averaged out or filtered out as shown in figure 26. The average gain for the pair of antennas is then determined as shown in figure 27. After processing the average gains obtained from the measurement of the three antenna pairs, the final gain values for the standard gain horns are determined. Some final results are shown in figures 28 and 29. The oscillations about the nominal values are approximately $\pm 0.04 \mathrm{~dB}, \pm 0.1 \mathrm{~dB}$, respectively, and are due to mismatches or multiple reflections at the throat and aperture of the horns. 


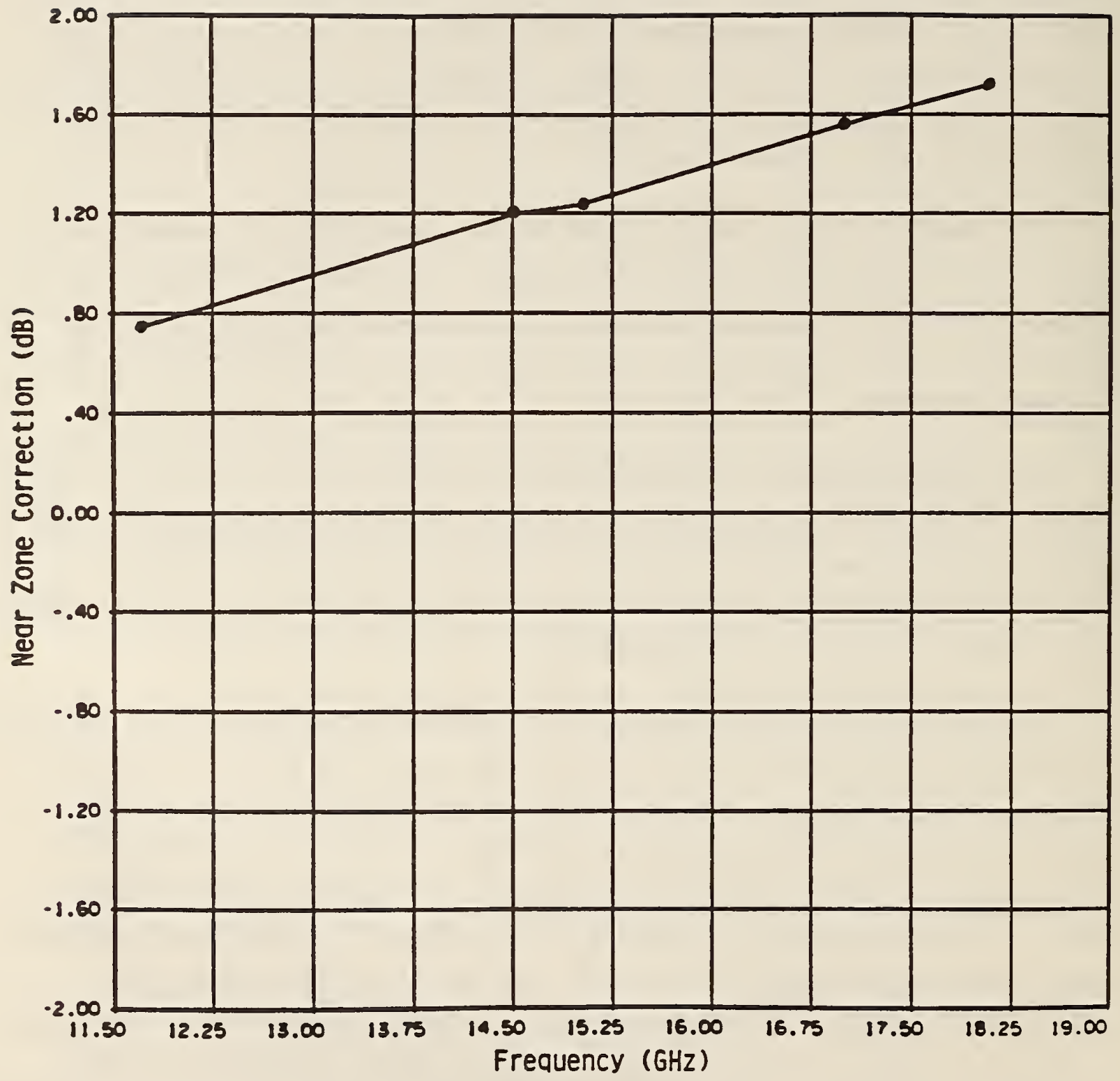

Figure 22. Near-zone correction obtained from extrapolation method for fixedfrequency measurements at $11.7,14.5,15.0,17.0$, and $18.1 \mathrm{GHz}$. 


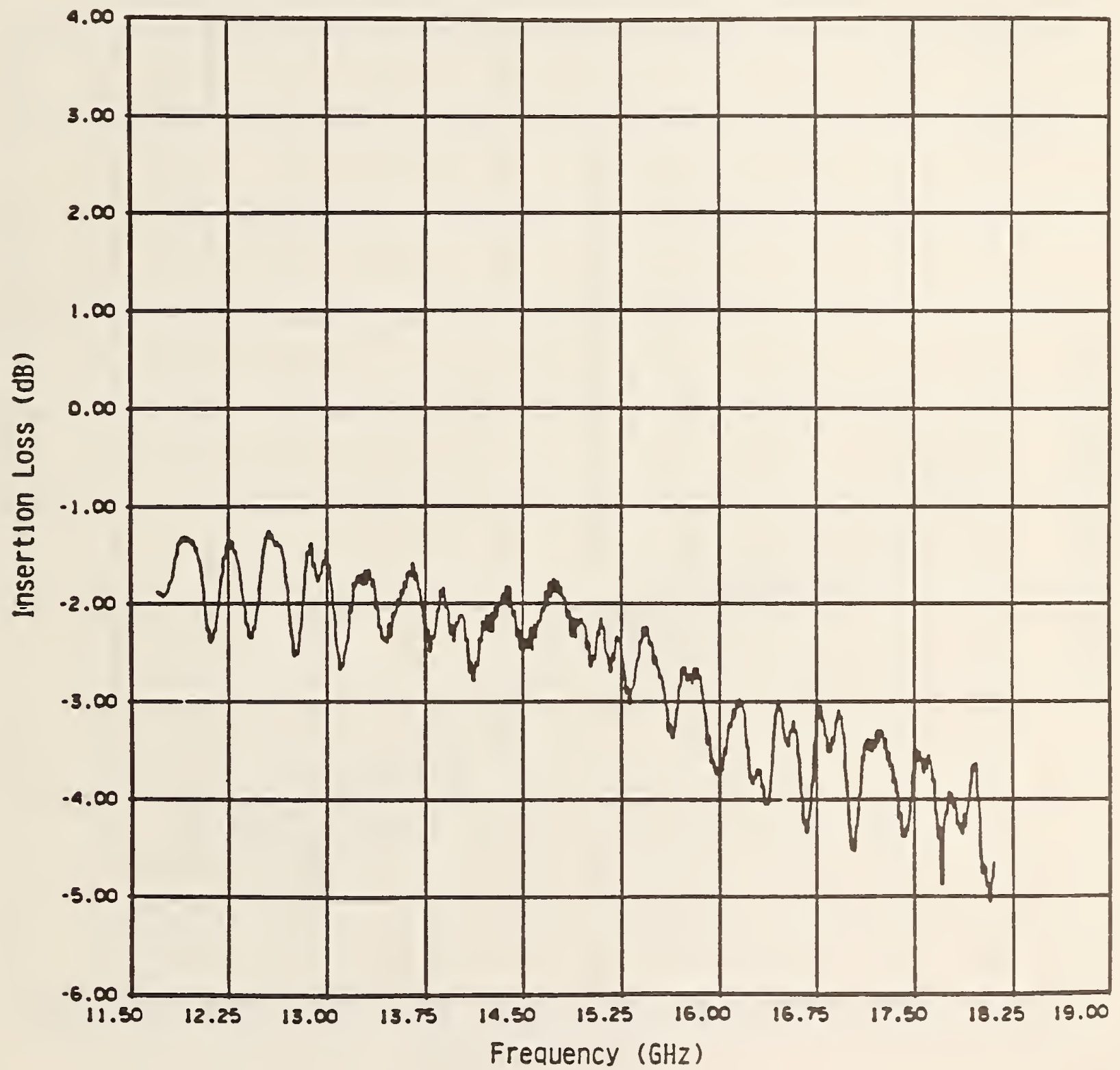

Figure 23. Swept frequency amplitude measurement with antennas in place at fixed distance of about $a^{2} / \lambda$. 


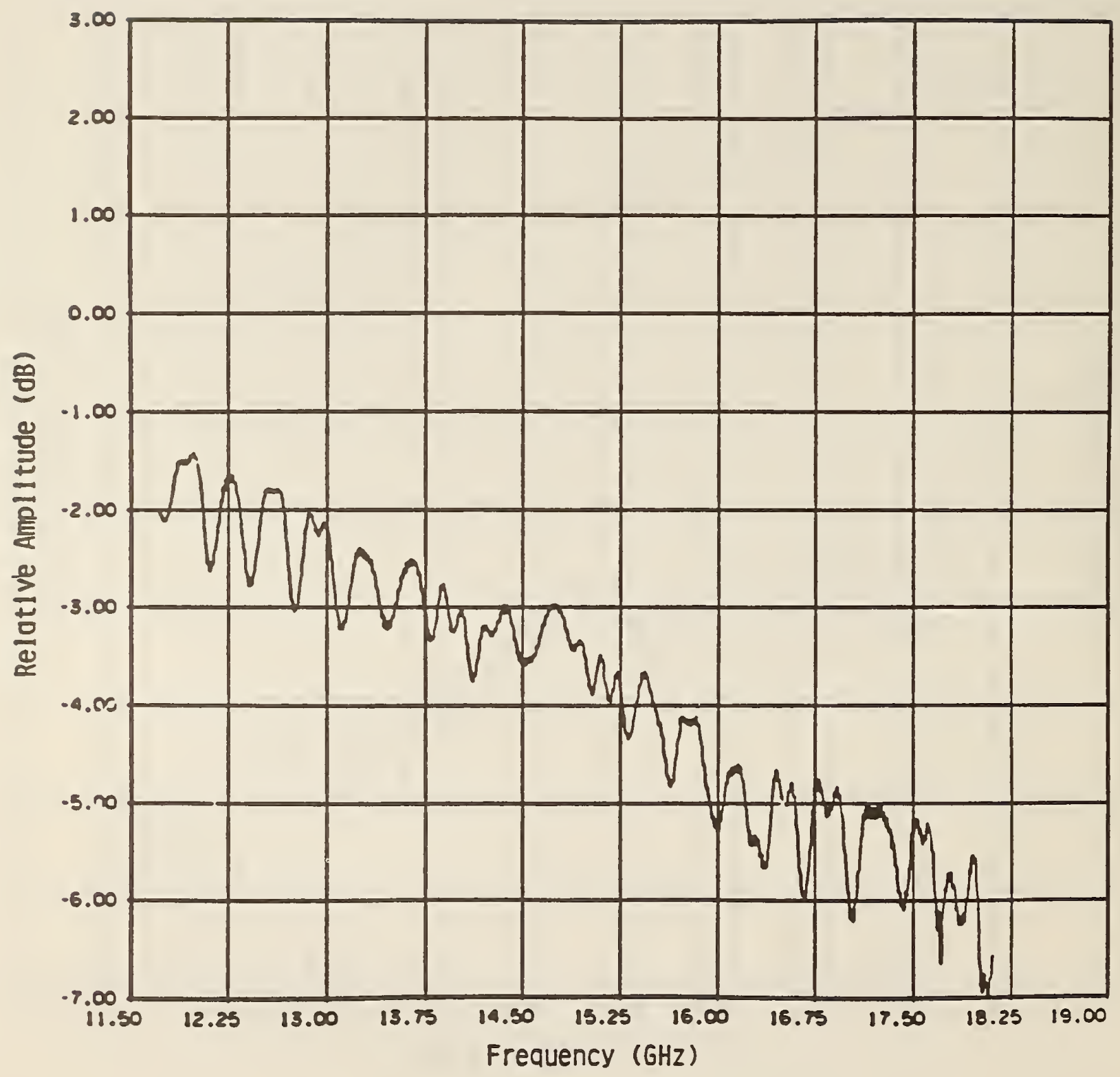

Figure 24. Swept frequency reference amplitude curve obtained with generator and load ports connected directly together. 


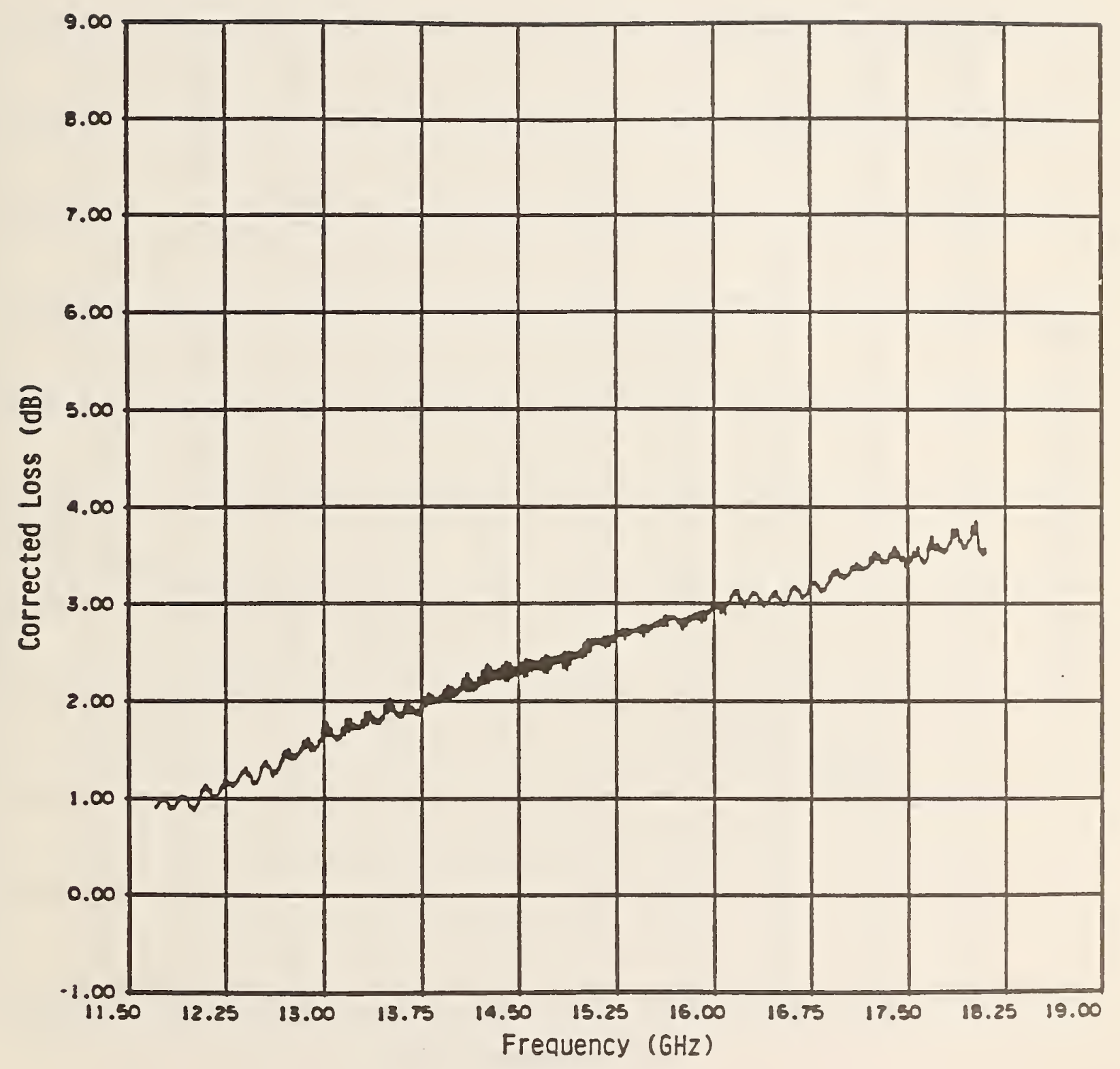

Figure 25. Insertion loss including mismatch and near-zone corrections. This is the difference between the reference curve and amplitude curve with antennas in place. The high-frequency oscillations are due to multiple reflections between the antennas. 


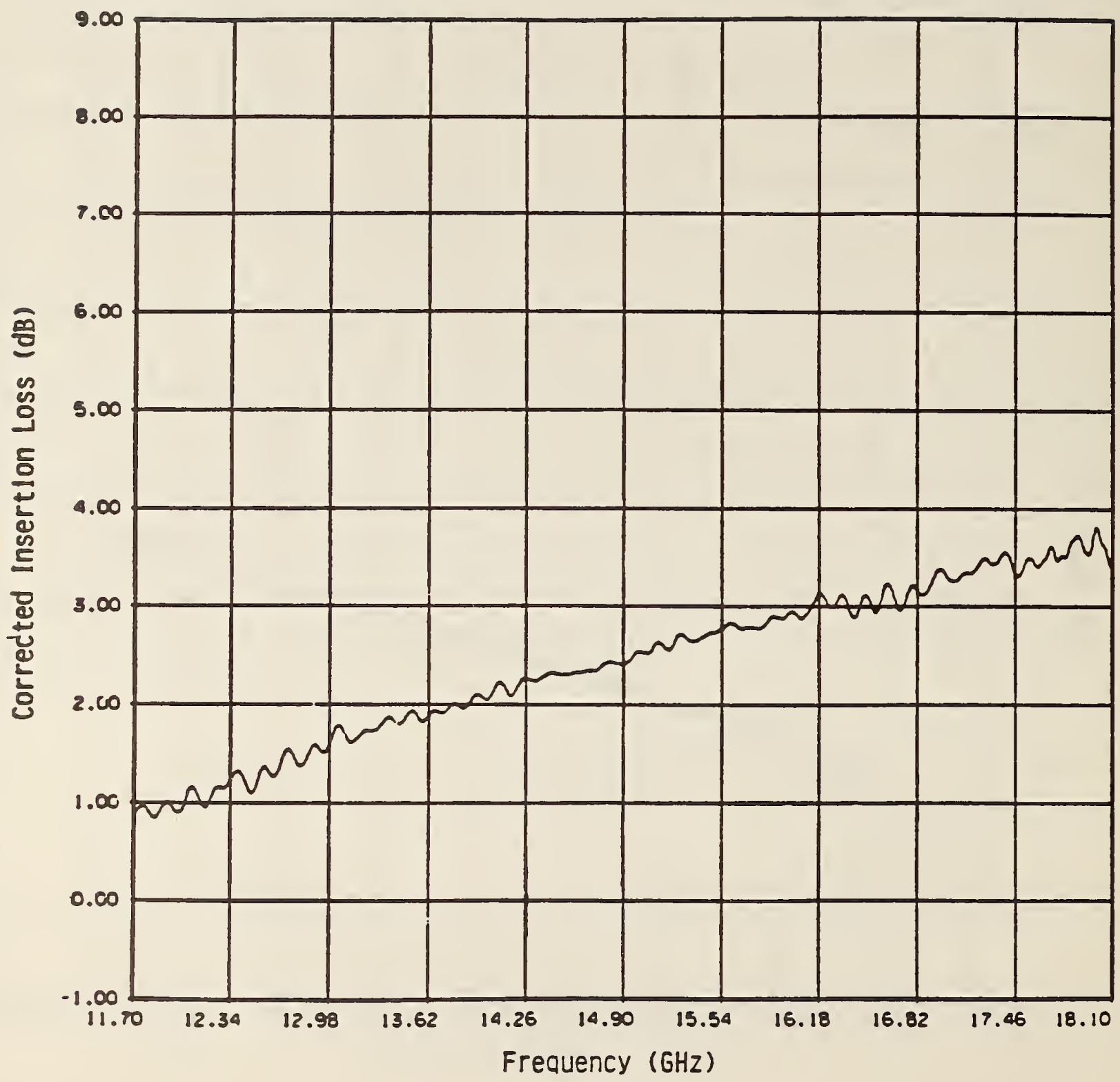

Figure 26. Insertion loss with multiple reflections averaged out. 


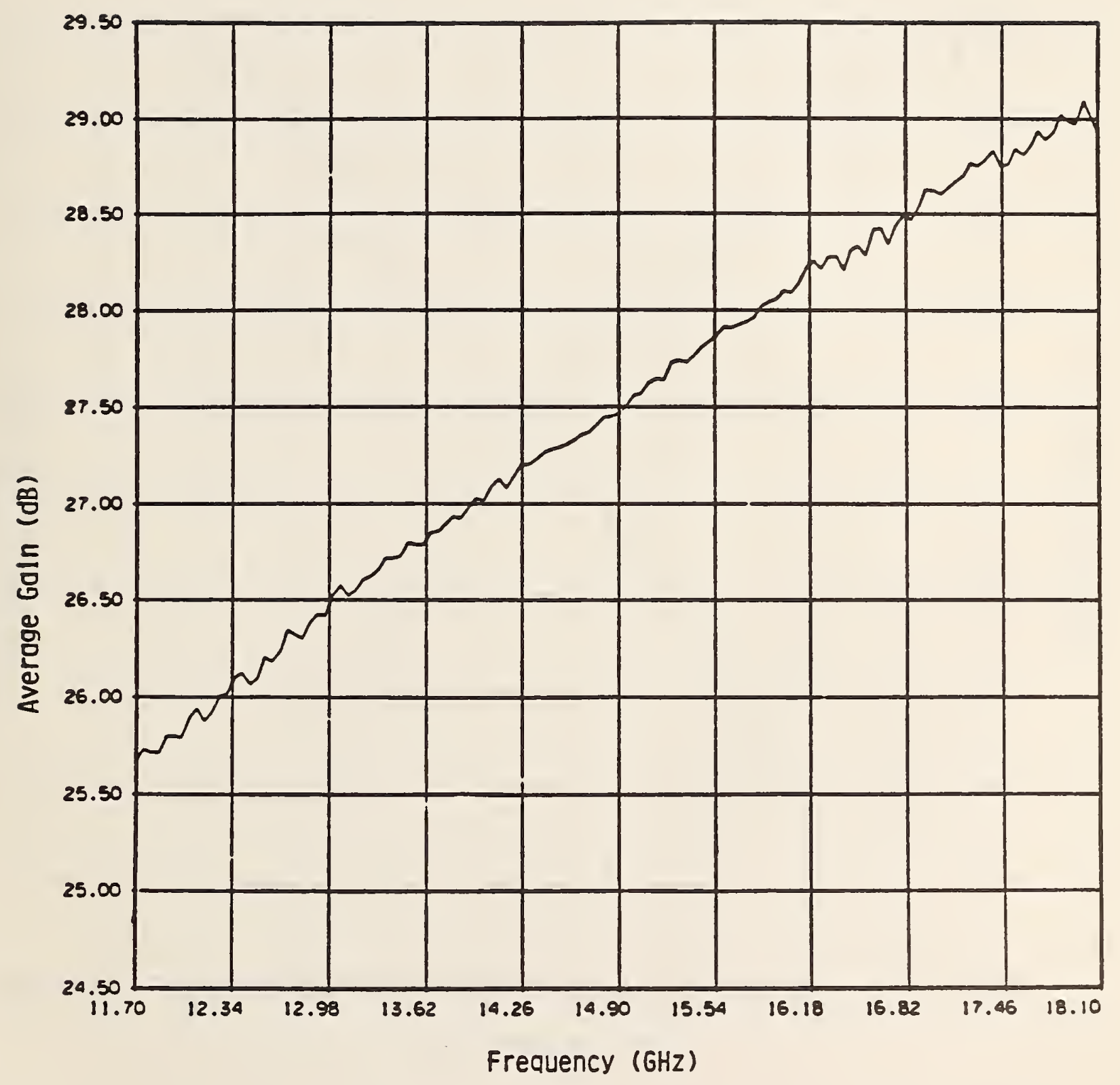

Figure 27. Average gain for a pair of antennas. 


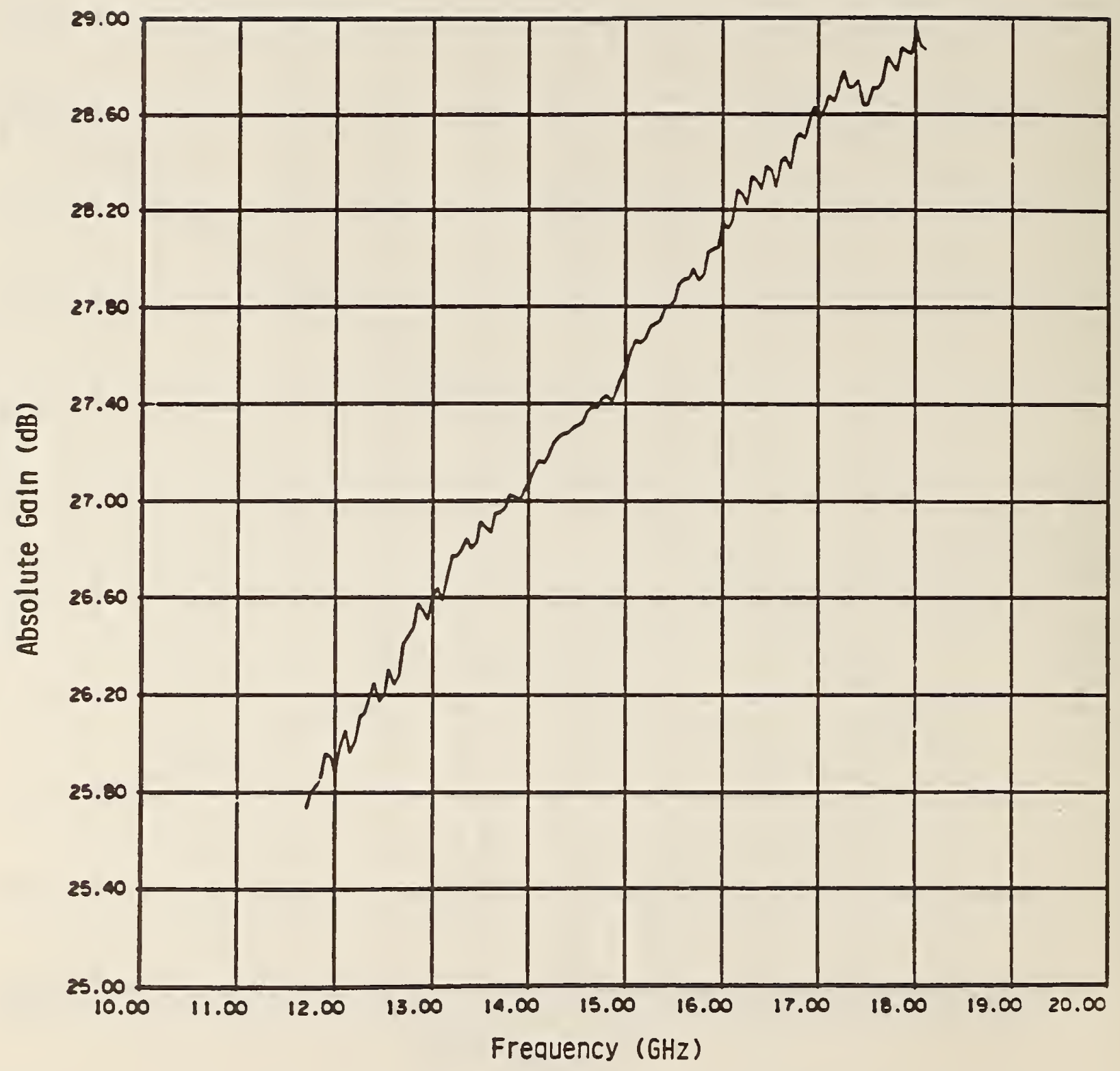

Figure 28. Final gain values for a long Ku-band horn. The oscillations about the nominal values are approximately $\pm 0.04 \mathrm{~dB}$ and are due to mismatches at the throat and aperture of the horn. 


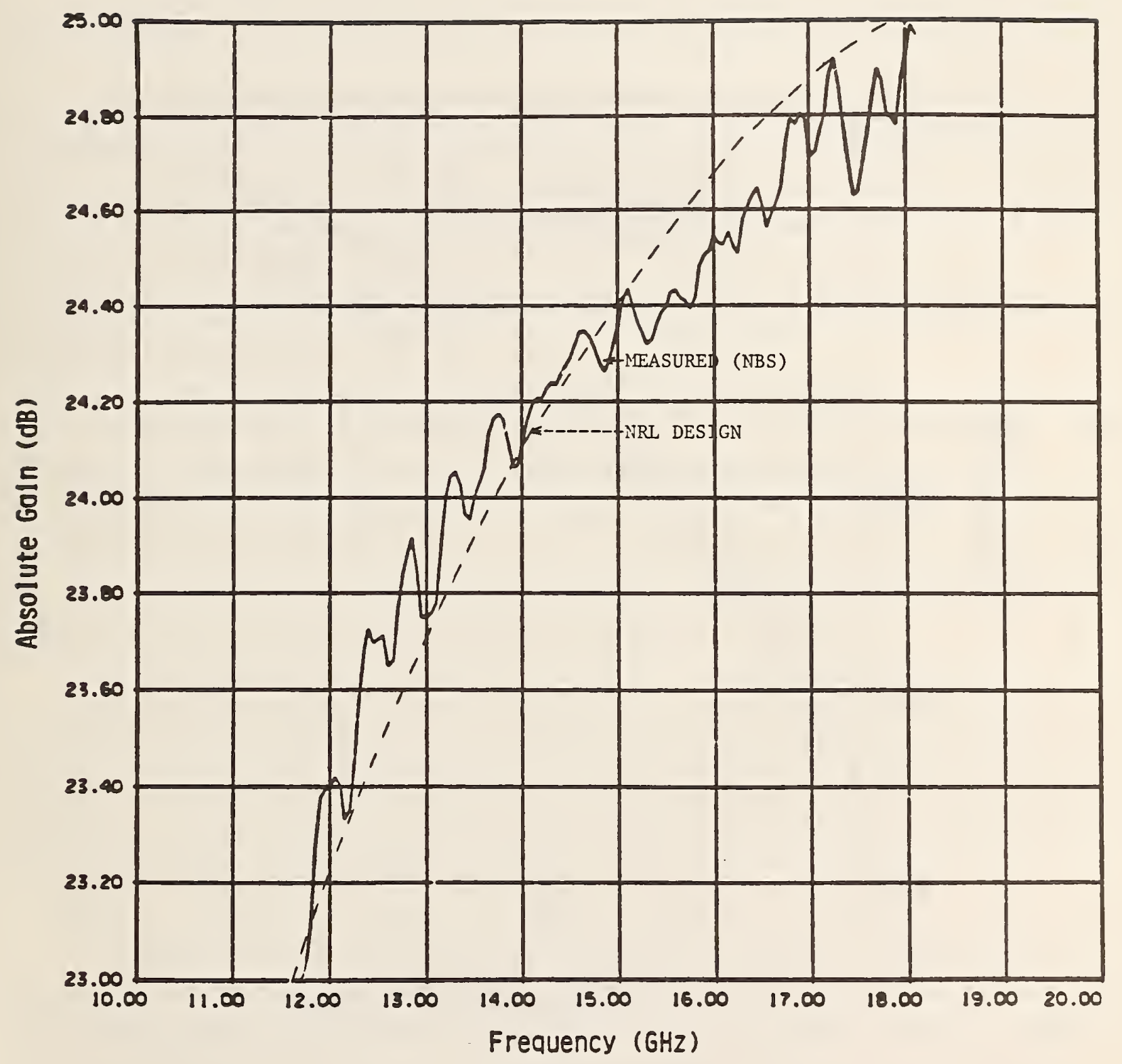

Figure 29. Final gain values for a standard gain horn. The oscillations about the nominal values are approximately $\pm 0.1 \mathrm{~dB}$ and are due to mismatches at the throat and aperture of the horn. 


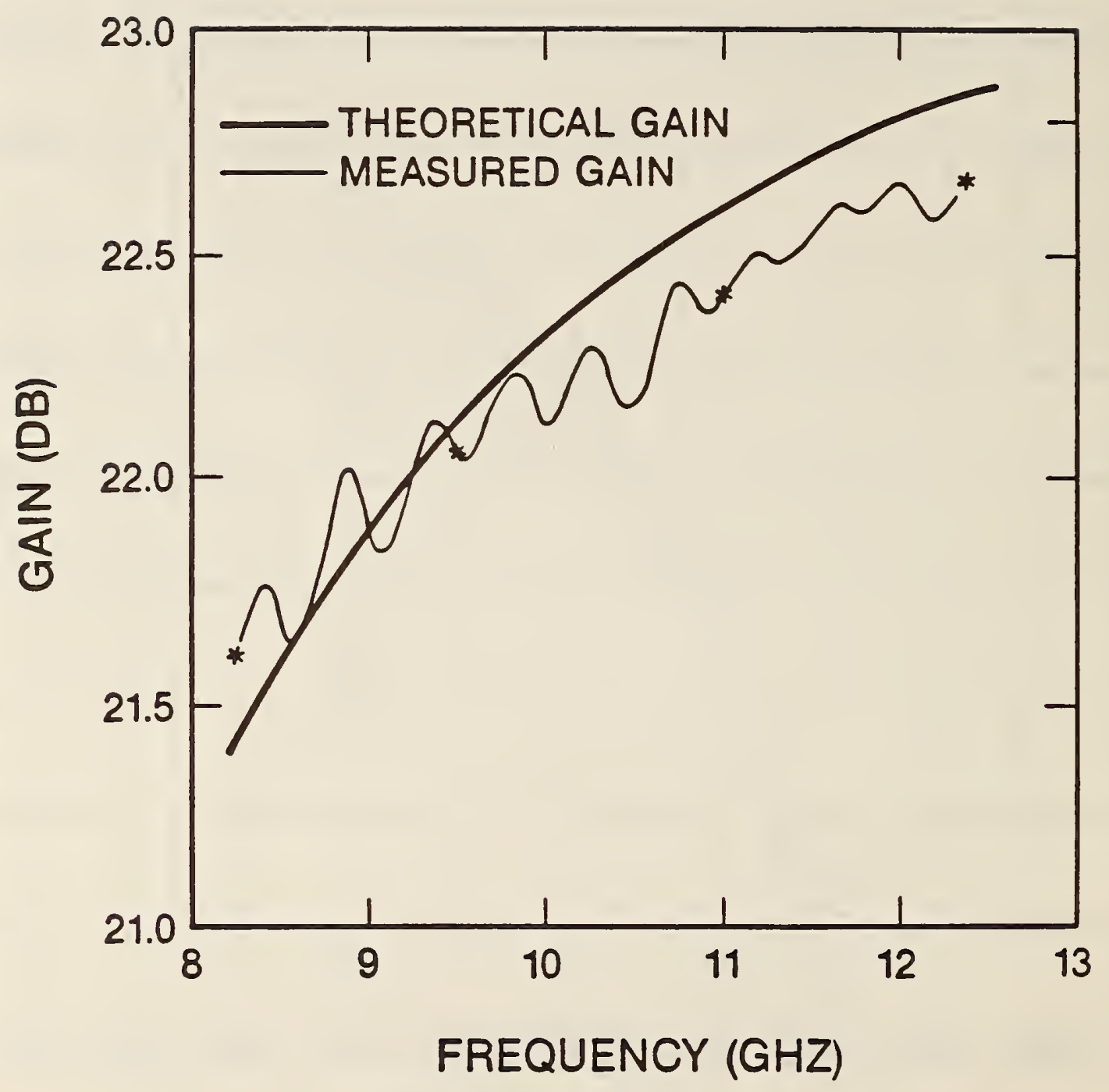

Figure 30. Comparison between theoretical gain and gain measured by the swept-frequency technique for an X-band pyramidal horn. Gain values indicated by the * were obtained by the extrapolation method. 
As another example, figure 30 illustrates a comparison between a theoretically obtained gain curve for an X-band pyramidal horn as predicted by theory [6] and data obtained using the three-antenna method applied to swept frequency data.

\section{Summary}

The extrapolation range measurement technique has been described for determining the power gain and polarization of antennas at reduced distances. It is used extensively by the National Bureau of Standards, Boulder, Colorado to calibrate antenna gain standards for industry and other agencies. A modification in the approach that significantly increases the accuracy of the polarization parameters has also been described and should be of particular interest to the reader.

Two extensions of the extrapolation method have also been presented. The first extension applies to swept frequency measurements where it has been demonstrated that accuracies over an entire band of frequencies can be achieved typically to within $\pm 0.15 \mathrm{~dB}$. Swept frequency measurements continue to be of interest to the measurement community as they can determine whether "holes" exist in an antenna's frequency response.

The second extension of the extrapolation method given as an appendix corrects for the effects of ground reflections in absolute gain measurements. This is particularly significant for broadbeam antennas such as those encountered below $1 \mathrm{GHz}$, where ground reflections may produce unwanted oscillations in the amplitude-versus-distance data.

In summary, the main purpose of this technical note is to describe the extrapolation measurement method, its improvements and its extensions, and its errors in detail. Through this exposition, it is hoped that managers, engineers, and technicians involved in antenna measurements will understand the deliberate steps necessary to ensure calibrations of the highest accuracy. 


\section{References}

[1] Kerns, D. M. Plane-wave scattering-matrix theory of antennas and antenna-antenna interactions. Nat. Bur. Stand. (U.S.) Monogr. 162; 1981 June.

[2] Leach, W. M., Jr.; Paris, D. T. Probe compensated near-field measurements on a cylinder. IEEE Trans. Antennas Propagat. AP-21: 435-445; 1973 July.

[3] Yaghjian, A. D. Near-field antenna measurements on a cylindrical surface: A source scattering-matrix formulation. Nat. Bur. Stand. (U.S.) Tech. Note 696; 1977 September.

[4] Jensen, F. Electromagnetic near-field far-field correlations. PhD. Dissertation, the Technical University of Denmark, Lyngby; 1970 June.

[5] Wacker, P. F. Non-planar near-field measurements: Spherical scanning. Nat. Bur. Stand. NBSIR 75-809; 1975 June.

[6] Monk, A. D. Pyramidal horn gain prediction and finite range correction. The Marconi Review. XLIV: 225-243; 1981 Fourth Quarter.

[7] Ju11, E. V. Errors in the predicted gain of pyramidal horns. IEEE Trans. Antennas Propagat. AP-21: 14-18; 1973 January.

[8] Hamid, M. A. K. Diffraction by a conical horn. IEEE Trans. Antenna Propagat. AP-16: 520-528; 1968 September.

[9] Chu, T. S.; Legg, W. E. Ga in of corrugated conical horns. IEEE Trans. Antennas Propagat. AP-30: 698-703; 1982 July.

[10] Bowman, R. R. Field strength above $1 \mathrm{GHz}$; measurement procedures for standard antennas. Proc. IEEE 55: 921-990; June 1967.

[11] Wacker, P. F. Theory and numerical techniques for accurate extrapolation of near-zone antenna and scattering measurements. Nat. Bur. Stand. (U.S.) unpublished report; 1972 April.

[12] Newell, A. C.; Kerns, D. M. Determination of both polarization and power gain of antennas by a generalized 3 -antenna measurement method. Electron. Lett. 7: 68-70; 1971 February 11.

[13] Newell, A. C.; Baird, R. C.; Wacker, P. F. Accurate measurement of antenna gain and polarization at reduced distances by an extrapolation technique. IEEE Trans. Antennas Propagat. AP-21(4): 418-431; 1973 July.

[14] Kerns, D. M. Definitions of $v, i, Z, Y, a, b, r$, and S. Proc. IEEE. 55: 892-900; 1967.

[15] Kerns, D. M.; Dayhoff, E. S. Theory of diffraction in microwave interferometry. Nat. Bur. Stand. (U.S.) J. Res. 64B: 1-13; 1960 JanuaryMarch. 
[16] Beatty, R. W. Discussion of errors in gain measurements of standard electromagnetic horns. Nat. Bur. Stand. (U.S.) Tech. Note 351; 1967 March.

[17] Hollis, J. S.; Lyon, T. J.; Clayton, L. Microwave antenna measurements. Atlanta, GA: Scientific Atlanta, Inc. 1970 July. p. 10-1 through 10-38.

[18] Hollis, J. S.; Lyon, T. J.; Clayton, L. Microwave antenna measurements. Atlanta, GA: Scientific Atlanta, Inc. 1970 July. p. 3-39 through 3-46.

[19] Anson, W. J. A guide to the use of the modified reflectometer technique of VSWR measurements. Nat. Bur. Stand. (U.S.) J. Res. Eng. Instr. 65C(4): 217-222; 1961 0ctober-December.

[20] Kerns, D. M.; Beatty, R. W. Basic theory of waveguide junctions and introductory microwave network analysis. Oxford, England: Pergamon Press; 1967.

[21] Natrella, M. G. Experimental statistics. Nat. Bur. Stand. (U.S.): Handb. 91, 1-8; 1963.

[22] Kenda11, M. G.; Stuart, A. The advanced theory of statistics, Vol. 2, Inference and relationship. New York: Hafner, 86; 1967.

[23] Wait, D. F.; Daywitt, W. C.; Kanda, M.; Miller, C. K. S. A study of the measurement of G/T using Cassiopeia A. Nat. Bur. Stand. (U.S.) NBSIR 74-382; 1974 0ctober.

[24] Kanda, M. Accuracy considerations in the measurement of the power gain of a large microwave antenna. Succinct papers, IEEE Trans. Antennas Propag.; 1975 May.

[25] Ludwig, A.; Hardy, J.; Norman, R. Gain calibration of a horn antenna using pattern integration. Jet Propulsion Lab., Pasadena, CA, Tech. Report 32-1572; 1972 0ctober.

[26] Chu, T. S.; Semplak, R. A. Gain of electromagnetic horns. Bell Syst. Tech. J. pp 527-537; 1965 March.

[27] Ippolito, L. J., Jr. Radiowave propagation in satellite communications. New York: Van Nostrand Reinhold Co.; 1986.

[28] Joy, E. B.; Paris, D. T. A practical method for measuring the complex polarization ratio of arbitrary antennas. IEEE Trans. Antennas Propag. AP-21: $432-435 ; 1973$ July.

[29] Newe11, A. C. Improved polarization measurements using a modified three antenna technique. AP-S Int. Symp. Digest: 337-340; 1975 June. 



\section{Appendix A. \\ Extension of Extrapolation Technique to Correct \\ for Effects of Ground Reflections}




\title{
Antenna Gain Measurements by an Extended Version of the NBS Extrapolation Method
}

\author{
ANDREW G. REPJAR, SENIOR MEMBER, IEEE, ALLEN C. NEWELL, MEMBER, IEEE, \\ AND RAMON C. BAIRD, SENIOR MEMBER, IEEE
}

\begin{abstract}
A General Extrapolation Technique which corrects for the effects of ground reflections in absolute gain measurements is described. It utilizes the Extrapolation Method developed at NBS which, in its present form, utilizes only amplitude versus distance data. However, for broadbeam antennas such as those encountered below $1 \mathrm{GHz}$, ground reflections may produce unwanted oscillations in the amplitude versus distance data. Hence the data are not amenable to the curve-fitting procedure of the Extrapolation Method. This problem can be overcome by including phase versus distance information to reduce the effects of ground reflections.
\end{abstract}

\section{INTRODUCTION}

A SPECIFIC OBJECTIVE of the Antenna Systems Metrology Group at NBS is to develop the required techniques and standards for accurately determining antenna characteristics. One existing NBS measurement service is the accurate calibration of transfer standard antennas (on-axis gain and polarization) by the Extrapolation Method. The theoretical basis of this method was developed by Wacker [1] and its application to accurate antenna measurements has been described briefly by Newell and Kerns [2] and more fully, in an experimentally oriented paper, by Newell et al. [3]. The method utilizes a generalized three-antenna approach which does not require quantitative a priori knowledge of the antennas.

The main advantages of the Extrapolation Method, which presently utilizes amplitude versus distance data only, are its accuracy and generality, and there is essentially no upper frequency limit. It can, in principle, be applied to any type of antenna although some directivity is desirable to reduce multipath interference. Above $1 \mathrm{GHz}$, uncertainties between \pm 0.08 and $\pm 0.10 \mathrm{~dB}$ in gain are achieved routinely. The maximum gain values that can be measured depend on the range dimensions since a maximum separation of approximately $2 D^{2} / \lambda$ is desirable. With the present NBS facilities, the maximum gain ranges from about $45 \mathrm{~dB}$ at $75 \mathrm{GHz}$ down to about $25 \mathrm{~dB}$ at $1 \mathrm{GHz}$.

At frequencies below $1 \mathrm{GH}$ z and for the purposes of this paper, in the VHF-UHF region, a transfer standard is often broadbeam and produces ground reflections which cause oscillations in the amplitude data as a function of distance. These oscillations have longer periods than those due to the multiple reflections between antennas which are routinely averaged out

Manuscript received August 19.1982.

The authors are with the Antenna Systems Metrolog! Section, Electromagnetic Ficlds Division. National Bureau of Standards. Boulder. CO 80303

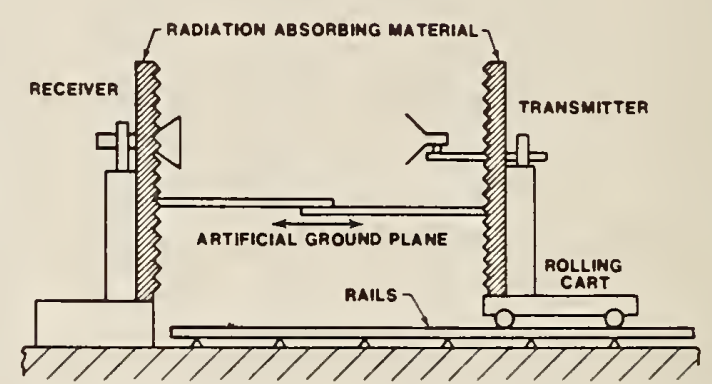

Fig. 1. Schematic diagram showing the NBS indoor extrapolation range modified to function as a scaled model of the larger outdoor range.

in the Extrapolation Method. For this reason, below $1 \mathrm{GHz}$ and for gains less than about $15 \mathrm{~dB}$, the antenna might not be calibratable by the usual Extrapolation Method which uses a curve-fitting procedure to determine gain. The purpose of this paper is to demonstrate that by utilizing both amplitude and phase data as a function of distance, one can obtain an amplitude versus distance curve which is corrected for the effects of ground reflections. This curve can then be processed by the Extrapolation Method to determine gain.

\section{Discussion of Measurement Procedure}

Recently at NBS, it was determined that a VHF-UHF scalar horn can be designed to meet the requirements of a transfer standard for $240-400 \mathrm{MHz}$ [4]. This fact was demonstrated by designing and constructing a $C$-band model of the VHF-UHF antenna and extensively measuring the critical performance parameters and comparing them with theoretical results. The expected calibration accuracy of the VHF-UHF horn was then determined by performing measurements of the scaled model on the NBS indoor extrapolation range. Measurements were conducted both with and without ground reflections so that we could determine how well we could correct for the multipath effects in our calibration technique. Ultimately, the full-scale horn will be measured on the NBS outdoor extrapolation range. Fig. 1 is an illustration of how we can use the scaled model horn on the indoor range to simulate the situation that will exist at $240-400 \mathrm{MHz}$ on the outdoor range.

\section{EXTRAPOLATION METHOD}

The first step in the measurement process is to connect the generator and load ports together to obtain a reference signal. The antennas are then installed and the received signal is re- 


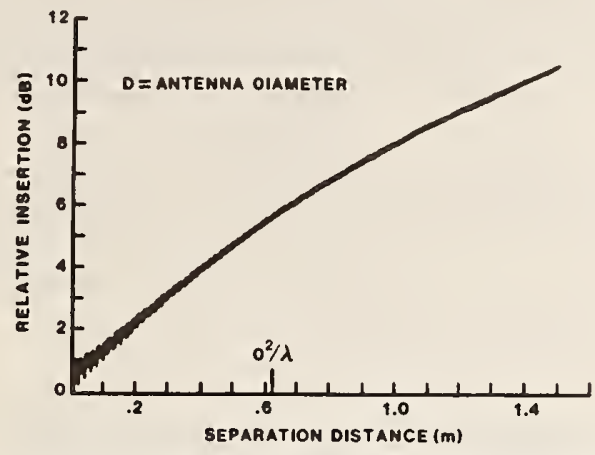

Fig. 2. Extrapolation data for scalar horn showing the insertion loss plotted as a function of separation distance (meters).

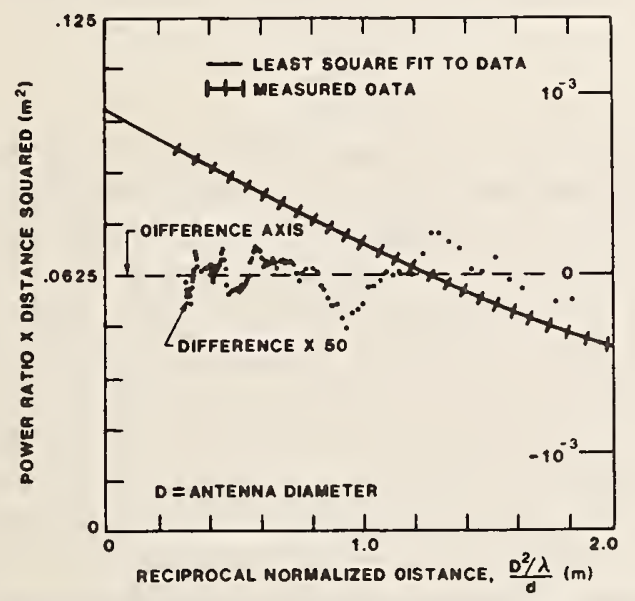

Fig. 3. Measured data without ground plane, four-term polynomial fit, and differences for scalar horn.

corded as a function of the separation distance. Fig. 2 displays some typical data for the case where no ground reflections are present. The oscillations are due to multiple reflections between the antennas. In the curve-fitting process to determine gain, the effects of these multiple transits are eliminated by locally averaging the relative insertion-loss values. The averaged curve is then fitted with a polynomia! in I/ $d$ (ordinarily, four or five terms will suffice). This procedure determines the coefficient, $A_{00}$, of the $1 / d$ term of the series (see [3], eq (27)] which determines the gain of the antenna under test. With the artificial ground plane removed and, hence, with no ground reflections present, the results obtained by the curve-fitting procedure are given in Fig. 3, which shows the averaged curve obtained from the measured data for the scalar horn and the curve obtained by fitting with a four-term polynomial. The greatly magnified differences between the measured and fitted curve qualitatively indicate the goodness of fit. The absolute gain of this horn was determined to be $13.32 \mathrm{~dB}$ at $4.0 \mathrm{GHz}$, which simulates 240 $\mathrm{MHz}$ in the VHF-UHF range. It should be noted that the abscissa in Fig. 3, and subsequently in Figs. 4 and 6, is the inverse of the normalized separation distance, a natural means of analyzing curve fitting with a polynomial in $1 / d$.

\section{ANALYSIS}

With the artificial ground plane in place, the averaged curve, i.e., the curve obtained after applying the local averaging process discussed above, as a function of antenna separation

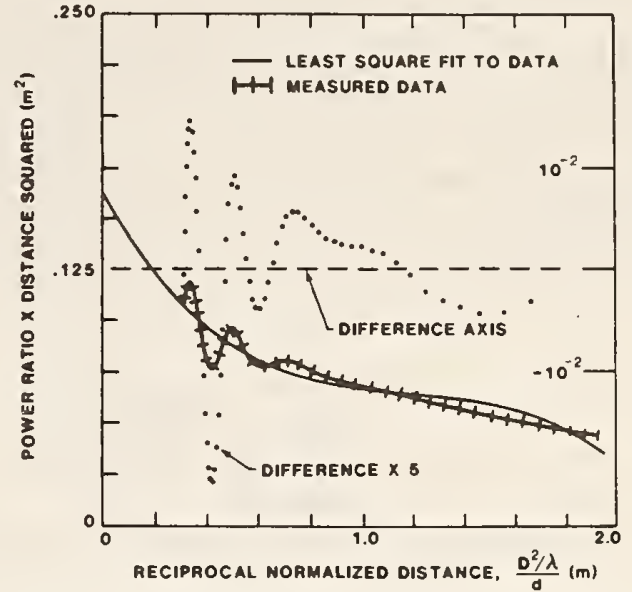

Fig. 4. Measured data with ground plane, four-term polynomial fit, and differences for scalar horn

is given in Fig. 4. Note the region of oscillatory behavior due to ground reflections. Fitting this curve with a four-term polynomial in $1 / d$, which is also shown in Fig. 4 , results in a calculated gain of $14.29 \mathrm{~dB}, 0.97 \mathrm{~dB}$ higher than its calibrated value obtained without the ground plane! In addition, it is evident from this figure that the differences between the averaged curve and the fitted curve are pronounced.

Denoting $\phi_{t}$ as the measured phase and $\phi_{d}$ as the phase of the direct wave, one can see that when the ground plane is removed, i.e., no ground effects present, the measured phase minus the phase of the direct wave should be zero plus an arbitrary constant in the far field. This is important in our analysis because the only assumption made in obtaining an amplitude versus distance plot that is free of multipath effects is that the direct wave phase can be represented in the far field as

$$
\phi_{d}=360 d / \lambda \text { degrees }
$$

where $d$ is the separation distance and $\lambda$ is the wavelength.

Using a laser interferometer to accurately measure the separation distance, the phase was measured without the ground plane to test the above assumption. (Since (1) is being used to calculate the phase of the direct wave, the measured separation distance must be accurate to about $1 / 360$ of a wavelength. For this reason, at $C$-band, a laser interferometer was used to measure the separation distances. For measurements of the full-scale UHF-VHF horn, other means may be adequate.) The dashed line in Fig. 5 shows the results, demonstrating that for large distances, $\phi_{t}-\phi_{d}$ is in fact extremely close to being constant. It should be noted that where the separation distance is small, the variation of $\phi_{t}-\phi_{d}$ is due to the near-zone effect, i.e., the antennas are still in the near field of each other. (A correction for this effect is routinely obtained in the standard extrapolation method.)

An analysis of the interaction between the direct wave and the ground wave shows that at the local peaks of the $\phi_{t}-\phi_{d}$ curve

$$
\rho \equiv \frac{\left|V_{g}\right|}{\left|V_{d}\right|}=\sin \left(\phi_{l}-\phi_{d}\right)
$$

where $\left|V_{g}\right|$ and $\left|V_{d}\right|$ are the amplitudes of the ground and direct waves, respectively. In addition, it can be shown that 


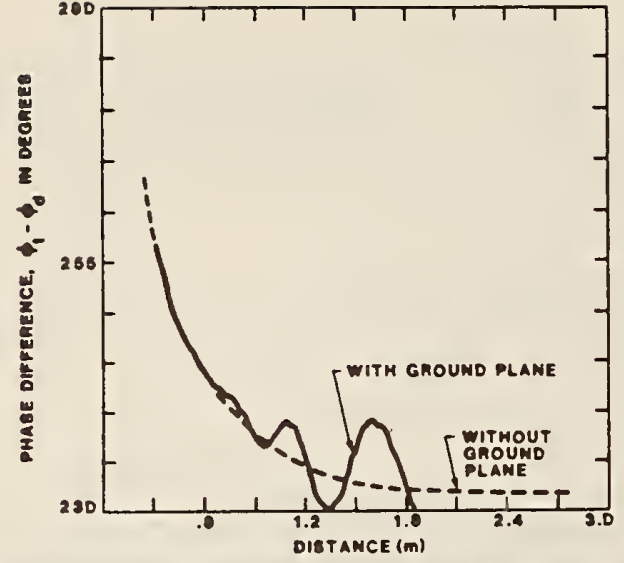

Fig. 5. Phase difference between measured wave and direct wave with and without ground plane.

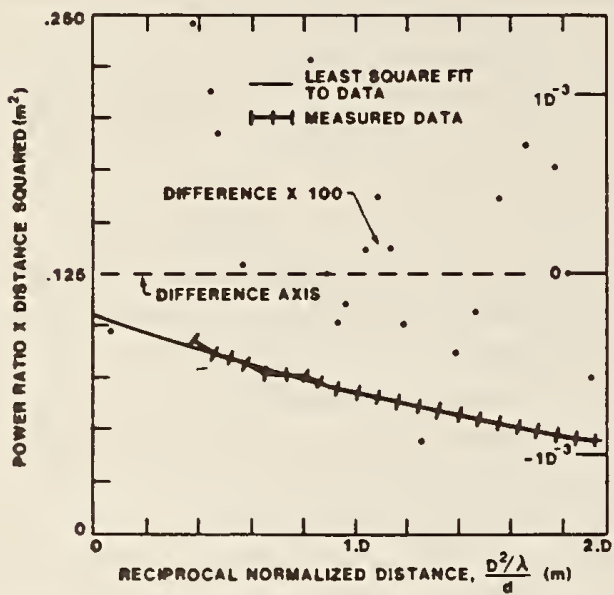

Fig. 6. Measured data points with ground plane and $\left|V_{l}\right|=\left|V_{d}\right|$. fourterm polynomial fit, and differences for scalar horn.

$$
\frac{\phi_{1}-\phi_{d}}{2}=\sin ^{-1}(\rho / 2), \quad \text { when }\left|V_{1}\right|=\left|V_{d}\right|
$$

where $\left|V_{l}\right|$ is the amplitude of the total wave resulting from the combination of the direct and ground waves. It is $\left|V_{l}\right|$ that is measured as a function of distance in the extrapolation method. Since $\left|V_{1}\right|_{\max }$ and $\left|V_{1}\right|_{\min }$ occur when the ground wave adds in and out of phase, respectively, with the direct wave, the distances at which $\phi_{t}-\phi_{d}=0$ are also determined. From these preceding results, the points on the insertion-loss curve where $\left|V_{l}\right|=\left|V_{d}\right|$ can be determined. For example, when the phase differences, $\phi_{l}-\phi_{d}$, are small, the condition $\left|V_{l}\right|=\left|V_{d}\right|$ occurs at those distances where the $\phi_{l}-\phi_{d}$ curve has local maxima and minima. Using only these points as data in the region where ground reflection effects are dominant, one can then use the extrapolation method to determine gain.

The solid line in Fig. 5 displays the measured $\phi_{l}-\phi_{d}$ data obtained with the ground plane in place. Through the above procedure, the points where $\left|V_{l}\right|=\left|V_{d}\right|$ were determined, and the curve obtained using these points in the region where ground effects are dominant is shown in Fig. 6. After fitting this curve with a four-term polynomial, also shown in Fig. 6, the gain of the antenna was calculated to be $13.27 \mathrm{~dB}$, only $0.05 \mathrm{~dB}$ low'er than the value obtained in the absence of ground reflections! In addition, in this figure, the differences between the curve and the fitted curve indicate the goodness of fit.
TABLE I

MEASUREd Gain in dB OF tHe SCALAR HORN MOdel (Principal component horizontally oriented.)

\begin{tabular}{cccc}
\hline $\begin{array}{c}\text { Frequency } \\
\text { in } \mathrm{GHz}\end{array}$ & Without ground ptane & With ground plene & $\begin{array}{c}\text { Whth ground diene } \\
\text { ind back-structure }\end{array}$ \\
\hline 4.0 & 13.32 & 13.27 & 1325 \\
4.83 & 1410 & 14.11 & 1415 \\
5.5 & 14.52 & 14.41 & 14.11 \\
6.8 & 12.15 & 12.17 & 12.22 \\
\hline
\end{tabular}

TABLE II

Measured Gain in dB of SCalar horn Model. (Principal component vertically oriented.)

\begin{tabular}{lccc}
\hline $\begin{array}{l}\text { Frequency } \\
\text { in GHz }\end{array}$ & Without ground plane & Whith ground plane & $\begin{array}{c}\text { With ground plane } \\
\text { and bock-structure }\end{array}$ \\
\hline 4.0 & 13.23 & 13.31 & 13.31 \\
4.83 & 14.09 & 14.02 & 14.03 \\
5.5 & 1441 & 14.50 & 1454 \\
6.8 & 12.17 & 12.26 & 12.29 \\
\hline
\end{tabular}

\section{RESULTS}

In order to further test the use of the extended version of the extrapolation method, the gain of the scaled-model antenna was determined at four frequencies across the band. The amplitude versus distance data obtained without the ground plane present were processed by the extrapolation method to determine the gain of the antenna at these frequencies. Next, the ainplitude versus distance data obtained with the ground plane present were processed by the extended version of the extrapolation method to determine the gain. Comparisons were then made. In addition, the gain of the antenna was obtained with its principal component first horizontally and then vertically oriented. (The phase of the field reflected by the ground plane depends on the polarization of the field incident on the ground plane. This results in different, i.e., shifted, positions for the oscillations in the amplitude versus distance data. To be useful, the extended version of the extrapolation method must give the correct gain for all orientations.) Finally, the structures of the towers on which antennas are mounted on the outdoor range were simulated to determine their effect on the gain measurements. The results of all these measurements are summarized in Tables I and II.

Upon examination of the data in Tables I and II, the rms difference between the gain values obtained with and without the ground plane are 0.07 and $0.09 \mathrm{~dB}$ for the principal component horizontally and vertically oriented, respectively. Also, the rms difference between the gain values obtained without the ground plane for the horizontal and vertical orientations is $0.07 \mathrm{~dB}$. These values are within $\pm 0.10 \mathrm{~dB}$, the bounds nominally expected by the error analysis discussed in [3]. It should be again noted that without the extended version of the extrapolation method to account for ground reflections, errors in gain could be in the order of \pm 1 to $2 \mathrm{~dB}$.

\section{CONCLUSIONS}

A technique has been described to determine the gain of antennas operating at frequencies below $1 \mathrm{GHz}$. Under simulated conditions, it was determined that phase versus distance measurements can be used to determine an amplitude versus distance curve which is corrected for ground reflection effects. This curve can then be processed by the extrapolation method which uses amplitude data only to determine gain. Measure- 
ments on the full-scale VHF-UHF horn on the NBS outdoor extrapolation range are planned for the future.

\section{REFERENCES}

[1] P. F. Wacker, "Theory and numerical techniques for accurate extrapolation of near-zone antenna and scattering measurements," NBS, Boulder, CO, unpublished Rep., Apr. 1972.

[2] A. C. Newell and D. M. Kerns, "Determination of both polarization and power gain of antennas by a generalized 3-antenna measurement method," Electron Lett., vol. 7. pp. 68-70, Feb. 11, 1971.

[3] A. C. Newell. R. C. Baird, and P. F. Wacker, "Accurate measurement of antenna gain and polarization at reduced distances by an extrapolation technique," IEEE Trans. Antennas Propagat., vol. AP-21, no. 4, pp. 418-431, July 1973.

[4] A. J. Estin, C. F. Stubenrauch, A. G. Repjar, and A. C. Newell, "Optimized wavelength-sized scalar horns as antenna radiation standards," IEEE Trans. Instrum. Meas., vol. IM-31, no. 1, pp. 53-56, Mar. 1982. 

Appendix B.

Standard NBS Calibration Report

B-1 


\title{
U.S. DEPARTMENT OF COMMERCE \\ NATIONAL BUREAU OF STANDARDS \\ NATIONAL ENGINEERING LABORATORY \\ Boulder, Colorado 80303
}

\section{REPORT OF CALIBRATION}

\author{
STANDARD GAIN HORN \\ National Bureau of Standards \\ X-Band Horn, Serial No. 485 \\ calibrated against \\ National Bureau of Standards \\ International Comparison Standard, Serial No. 1 \\ and \\ National Bureau of Standards \\ International Comparison Standard, Serial No. 2
}

Submitted by:

National Bureau of Standards

Antenna Systems Metrology Group

Boulder, Colorado 80303

The on-axis power gain and polarization parameters of the antenna were measured by a generalized three antenna measurement technique [1]. An extrapolation measurement technique was used to evaluate and correct for near-zone and multipath effects in the measured data. The two other antennas which were used in the measurement were pyramidal type horns with nominal linear polarization. The antennas were not assumed to be perfectly linearly polarized, and their actual cross polarization was accounted for in the measurements and calculations.

The axis of measurement was in the direction of maximum power gain for all antennas, and their ' $E$ ' field was in the horizontal direction.

The results of the measurements are summarized in the following table.

\begin{tabular}{cccccccc}
\hline $\begin{array}{c}\text { Frequency } \\
(\mathrm{GHz})\end{array}$ & $\begin{array}{c}\text { Power Gain } \\
(\mathrm{dB})\end{array}$ & $\begin{array}{c}A x i a 1 \text { Ratio } \\
(\mathrm{dB})\end{array}$ & $\begin{array}{c}\text { Sense of } \\
\text { Polarization }\end{array}$ & $\begin{array}{c}\text { Tilt Angle } \\
\text { (degrees) }\end{array}$ & \multicolumn{2}{c}{ Reflection Coefficient } \\
\cline { 5 - 8 } & $21.60 \pm 0.1$ & $48 \pm 2$ & Right & $90.3 \pm 0.5$ & 0.049 & 105.9 \\
10.00 & $21.37 \pm 0.1$ & $44 \pm 2$ & Right & $90.1 \pm 0.5$ & 0.043 & -59.6 \\
12.00 & $22.78 \pm 0.1$ & $43 \pm 2$ & Right & $90.3 \pm 0.5$ & 0.025 & -147.9 \\
\hline
\end{tabular}

In addition to the gain measurements at the fixed frequencies, swept gain measurements were also obtained. The results of these measurements are shown in figure 1.

[1] Newe11, A. C.; Baird, R. C.; Wacker, P. F. Accurate measurement of antenna gain and polarization at reduced distances by an extrapolation technique. IEEE

Trans. Antennas and Propagation, Vol. AP-21:418-431; 1973 July.

Page 1 of 2

Date of Calibration: July 1982 
Standard Gain Horn

National Bureau of Standards

X-Band Horn, Serial No. 485

calibrated against

National Bureau of Standards

International Comparison Standard, Serial No. 1

and National Bureau of Standards

International Comparison Standard, Serial No. 2

The error estimates are the result of a quadrature (RSS) sum of the individual error components listed in the following tables. Each error component is a worst case or 3 sigma value, and the errors are independent and uncorrelated. The confidence level for the resulting combination is, therefore, equivalent to that associated with 3 sigma.

Errors in Gain Measurement

Source of Error

System Drift and Receiver Nonlinearity

Attenuator Calibration

Impedance Mismatch

Antenna Alignment

Distance Nonlinearity

Flange Loss and Reproducibility

Residual Multipath

Random Errors
Resultant Error in Gain (dB)

0.05

0.04

$<0.01$

0.05

0.02

0.05

0.03

0.03

Quadrature Sum $\quad \pm 0.11$

Errors in Polarization Measurements

Source of Error

Antenna Alignment

Residual Multipath

Rotation Angle for Maximum and Minimum
Axial ratio (dB) Tilt angle (deg.)

\begin{tabular}{ll}
1.0 & 0.5 \\
1.0 & 0.1 \\
1.5 & 0.15 \\
\hline 2.0 & \pm 0.5
\end{tabular}

The estimated uncertainty in the swept gain measurement is $0.15 \mathrm{~dB}$.

For the Director,

National Engineering Laboratory

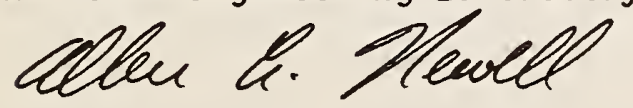

Allen C. Newel1, Leader

Antenna Systems Metrology Group

Electromagnetic Fields Division

Page 2 of 2

Date of Calibration: July 1982 
INT. CONP. STD. XTR

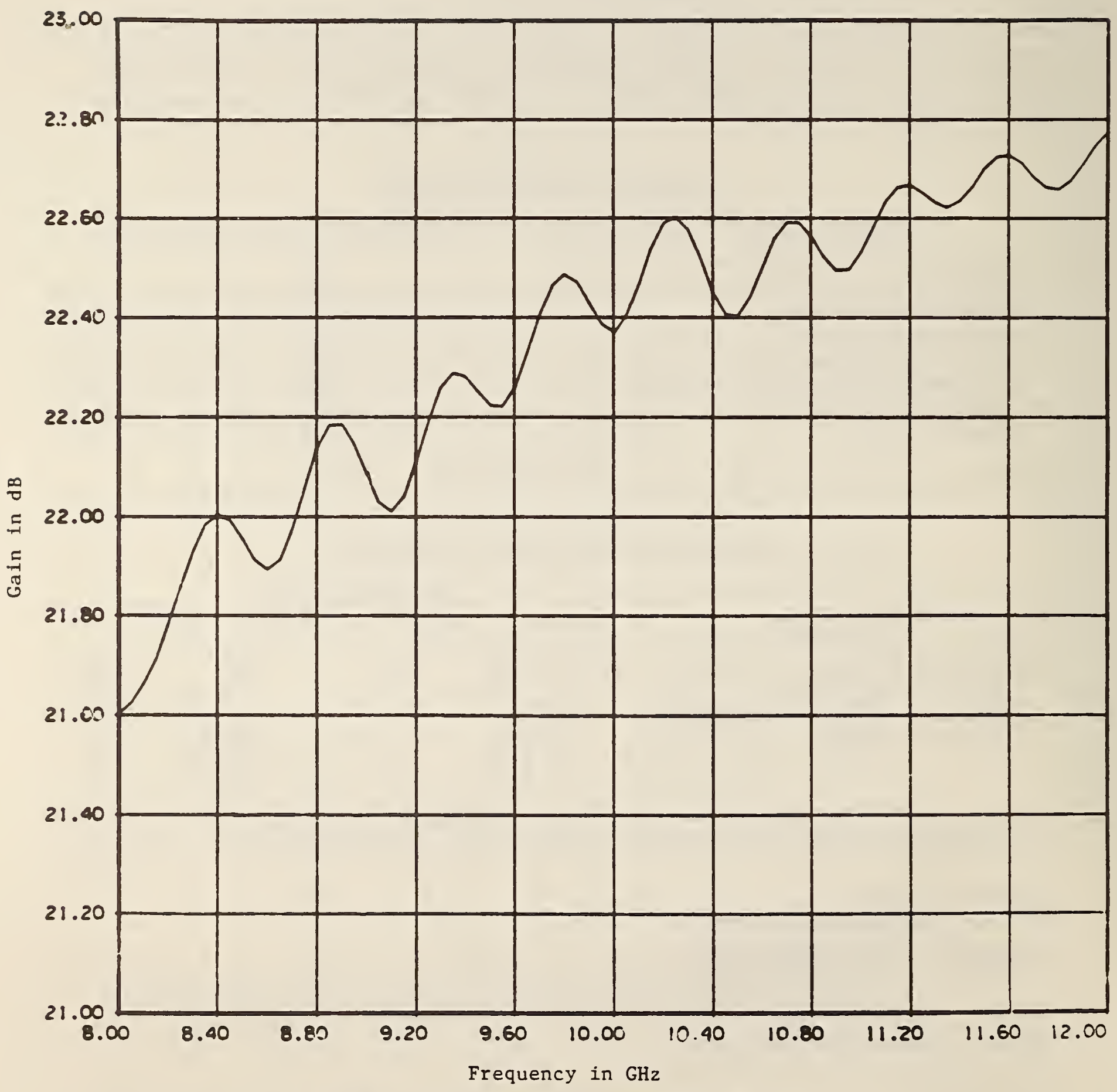

Figure 1. NBS X-Band Horn, Serial No. 485. 
NBS-114A (REV. 2-8C)

\begin{tabular}{|c|c|c|c|}
\hline $\begin{array}{l}\text { U.5. DEPT. OF COMM. } \\
\text { BIBLIOGRAPHIC DATA } \\
\text { SHEET (See instructions) }\end{array}$ & $\begin{array}{l}\text { 1. PUBLICATION OR } \\
\text { REPORT NO. } \\
\text { NBS/TN-1311 }\end{array}$ & 2. Performing Organ. Report No. & $\begin{array}{l}\text { 3. Publication Date } \\
\text { August } 1988\end{array}$ \\
\hline \multicolumn{3}{|c|}{ Extrapolation Range Measurements for Determining Antenna Gain and Polarization } & and Polarization \\
\hline \multicolumn{4}{|c|}{$\begin{array}{l}\text { 5. AuTHOR(S) } \\
\text { Andrew G. Repjar, Allen C. Newell, and Douglas T. Tamura }\end{array}$} \\
\hline \multicolumn{3}{|c|}{$\begin{array}{l}\text { 6. PERFORMING ORGANIZATION (If joint or other than NBS, see instructions) } \\
\text { NATIONAL BUREAU OF STANDARDS } \\
\text { DEPARTMENT OF COMMERCE } \\
\text { WASHINGTON, D.C. } 20234\end{array}$} & 8. Type of Report \& Period Covered \\
\hline \multicolumn{4}{|c|}{ 9. SPONSORING ORGANIZATION NAME AND COMPLETE ADDRESS (Street. City. State. ZIP) } \\
\hline
\end{tabular}

10. SUPPLEMENTARY NOTES

[Document describes a computer program; SF-185, FIPS Software Summary, is attached.

11. ABSTRACT (A 200-word or less factual summary of most significant information. If document includes a significant bibliography or literature survey. mention it here)

The extrapolation range measurement technique for determining the power gain and polarization of antennas at reduced range distances is described. It is based on a generalized three-antenna approach and does not require quantitative a priori knowledge of the antennas. During the past decade, it has been extensively used by the National Bureau of Standards, Boulder, Colorado, to calibrate antenna gain standards for industry and other agencies within $\pm 0.1 \mathrm{~dB}$. To help one understand how calibrations of this accuracy are achieved, the extrapolation range description includes discussions on the required theory, the measurement procedures, the range configuration and instrumentation, the errors, and some measurement examples. Recent extensions of the extrapolation method required for swept/stepped frequency gain calibrations and for corrections to reduce ground reflection effects, are also presented.

12. KEY WORDS (Six to twelve entries; alphabetical order; capitalize only proper names; and separate key words by semicolons) antenna measurements; antenna ranges; antennas; near-field measurements; probe antennas standard gain horns; swept frequency measurements

13. AVAILABILITY

$\mathrm{X}$ Unlimited

For Official Distribution. Do Not Release to NTIS

[X] Order From Superintendent of Documents, U.S. Government Printing Office, Washington, D.C. 20402.

14. NO. OF

PRINTED PAGES

38

15. Price

— Order From National Technical Information Service (NTIS), Springfield, VA. 2216I 



\section{Periodical}

Journal of Research-The Journal of Research of the National Bureau of Standards reports NBS research and development in those disciplines of the physical and engineering sciences in which the Bureau is active. These include physics, chemistry, engineering, mathematics, and computer sciences. Papers cover a broad range of subjects, with major emphasis on measurement methodology and the basic technology underlying standardization. Also included from time to time are survey articles on topics closely related to the Bureau's technical and scientific programs. Issued six times a year.

\section{Nonperiodicals}

Monographs-Major contributions to the technical literature on various subjects related to the Bureau's scientific and technical activities.

Handbooks-Recommended codes of engineering and industrial practice (including safety codes) developed in cooperation with interested industries, professional organizations, and regulatory bodies.

Special Publications - Include proceedings of conferences sponsored by NBS, NBS annual reports, and other special publications appropriate to this grouping such as wall charts, pocket cards, and bibliographies.

Applied Mathematics Series-Mathematical tables, manuals, and studies of special interest to physicists, engineers, chemists, biologists, mathematicians, computer programmers, and others engaged in scientific and technical work.

National Standard Reference Data Series-Provides quantitative data on the physical and chemical properties of materials, compiled from the world's literature and critically evaluated. Developed under a worldwide program coordinated by NBS under the authority of the National Standard Data Act (Public Law 90-396).

NOTE: The Journal of Physical and Chemical Reference Data (JPCRD) is published quarterly for NBS by the American Chemical Society (ACS) and the American Institute of Physics (AIP). Subscriptions, reprints, and supplements are available from ACS, 1155 Sixteenth St., NW, Washington, DC 20056.

Building Science Series-Disseminates technical information developed at the Bureau on building materials, components, systems, and whole structures. The series presents research results, test methods, and performance criteria related to the structural and environmental functions and the durability and safety characteristics of building elements and systems.

Technical Notes-Studies or reports which are complete in themselves but restrictive in their treatment of a subject. Analogous to monographs but not so comprehensive in scope or definitive in treatment of the subject area. Often serve as a vehicle for final reports of work performed at NBS under the sponsorship of other government agencies.

Voluntary Product Standards--Developed under procedures published by the Department of Commerce in Part 10, Title 15, of the Code of Federal Regulations. The standards establish nationally recognized requirements for products, and provide all concerned interests with a basis for common understanding of the characteristics of the products. NBS administers this program as a supplement to the activities of the private sector standardizing organizations.

Consumer Information Series-Practical information, based on NBS research and experience, covering areas of interest to the consumer. Easily understandable language and illustrations provide useful background knowledge for shopping in today's technological marketplace.

Order the above NBS publications from: Superintendent of Documents, Government Printing Office, Washington, DC 20402.

Order the following NBS publications-FIPS and NBSIR's-from the National Technical Information Service, Springfield, VA 22161.

Federal Information Processing Standards Publications (FIPS PUB)-Publications in this series collectively constitute the Federal Information Processing Standards Register. The Register serves as the of ficial source of information in the Federal Grovernment regarding standards issued by NBS pursuant to the Federal Property and Administrative Services Act of 1949 as amended, Public Law 89-306 (79 Stat. 1127), and as implemented by Executive Order 11717 (38 FR 12315, dated May 11, 1973) and Part 6 of Title 15 CFR (Code of Federal Regulations).

NBS Interagency Reports (NBSIR)-A special series of interim or final reports on work performed by NBS for outside sponsors (both government and non-government). In general, initial distribution is handled by the sponsor; public distribution is by the National Technical Information Service, Springfield, VA 22161, in paper copy or microfiche form. 
U.S. Department of Commerce

National Bureau of Standards

Gaithersburg, MD 20899

Official Business

Penalty for Private Use $\$ 300$ 